Illinois State University

ISU ReD: Research and eData

Theses and Dissertations

$6-6-2014$

\title{
Examination of Quasienantiomers of Oxadiazinanones Which Fail to Fractionally Crystallize
}

Patricia Ann Higgins

Illinois State University, pahiggins3@comcast.net

Follow this and additional works at: https://ir.library.illinoisstate.edu/etd

Part of the Chemistry Commons

\section{Recommended Citation}

Higgins, Patricia Ann, "Examination of Quasienantiomers of Oxadiazinanones Which Fail to Fractionally Crystallize" (2014). Theses and Dissertations. 203.

https://ir.library.illinoisstate.edu/etd/203

This Thesis is brought to you for free and open access by ISU ReD: Research and eData. It has been accepted for inclusion in Theses and Dissertations by an authorized administrator of ISU ReD: Research and eData. For more information, please contact ISUReD@ilstu.edu. 


\section{EXAMINATION OF QUASIENANTIOMERS OF \\ OXADIAZINANONES WHICH FAIL TO \\ FRACTIONALLY CRYSTALLIZE}

\section{Patricia A. Higgins}

86 Pages

August 2014

This thesis reports the crystallization of two new quasiracemates, the synthesis of a new oxadiazinanone and the identification of crystals of two new compounds. In previous research the quasiracemates, (5R,6S )-4-cyclohexyl-5-methyl-6-phenyl-2H1,3,4-oxadiazinan-2-one and (5S,6R)-4-isopropyl-5-methyl-6-phenyl-2H-1,3,4oxadiazinan-2-one, were mixed in solution and crystallized. Instead of fractionally crystallizing, as would be expected, the compounds formed quasiracemic co-crystals. This suggested that other mixtures of oxadiazinanones would be possible candidates for studying compounds which fail to fractionally crystallize.

The first step in the process involves the synthesis of oxadiazinanones, using literature methods. A 4-step process is employed involving alkylation, nitrosation, reduction and cyclization. Because the starting material, norephedrine, is a chiral molecule, the desired enantiomer is synthesized starting with the proper enantiomer of norephedrine-either the $(1 S, 2 R)$ or $1(R, 2 S)$ enantiomers. A new oxadiazinanone, (5R,6S)-4-cyclopentyl-5-methyl-6-phenyl-2H-1,3,4-oxadiazinan-2-one, was synthesized. After synthesis, the oxadiazinanones are mixed together and crystallized using vapor 
diffusion. X-ray diffraction is used to study the structures to determine the presence of quasiracemates.

From the sixteen racemic pairs compared, it was found that quasiracemates were formed from oxadiazinanones containing either an $\mathrm{N}_{4}$-cyclopentyl substituent or an $\mathrm{N}_{4}$ cyclohexyl substituent. The quasiracemate formed has a lower $Z$ ' than at least one of the quasienantiomers in the structure.

Two additional compounds were synthesized, and the crystal structures were identified . These crystals were obtained when the intended $\mathrm{N}_{4}$-ethylated and $\mathrm{N}_{4}-n$ butylated oxadiazinanones failed to cyclize in the final step of the oxadiazinanone synthesis. 
EXAMINATION OF QUASIENANTIOMERS OF

OXADIAZINANONES WHICH FAIL TO

FRACTIONALLY CRYSTALLIZE

PATRICIA A. HIGGINS

A Thesis Submitted in Partial

Fulfillment of the Requirements for the Degree of

MASTER OF SCIENCE

Department of Chemistry

ILLINOIS STATE UNIVERSITY

2014 
(C) 2014 Patricia A. Higgins 


\title{
EXAMINATION OF QUASIENANTIOMERS OF OXADIAZINANONES WHICH FAIL TO FRACTIONALLY CRYSTALLIZE
}

\author{
PATRICIA A. HIGGINS
}

COMMITTEE MEMBERS:

Gregory M. Ferrence, Chair

Christopher G. Hamaker

Shawn R. Hitchcock 


\section{ACKNOWLEDGMENTS}

I would like to thank my advisor, Dr. Greg Ferrence, for his help and guidance through anxieties, tears, and everything else this degree entailed. Dr. Shawn Hitchcock provided a tremendous amount of time and great patience in helping me with the syntheses of my oxadiazinanones and for this I am very grateful! Dr. Christopher Hamaker provided help with the XRD and served as one of my committee members and I am very thankful for his support as well. The financial support in the form of assistantships from Illinois State University was appreciated.

My husband, Art, has been a rock! My children, Christy and Dan, Chelsea and Alan, Matt and Morgan, Julia and Adam, and Annie have been a source of encouragement during the process. The arrival of Asher, my precious grandson, was a source of joy in the midst of hard work. Much gratitude goes to Dr. Nagorski and Sarah Stewart who made it possible for me to be there when Asher made his arrival.

Dr. Sharon Weldon provided a home, deep friendship and valuable advice and I am grateful. Sharon, I look forward to many years of friendship ahead!

My friends in the Millikin University Department of Chemistry have given me strong encouragement. Anne, Clarence, Ed, George, Paris, Lynette and Kate-I am so thankful for each of you! 


\section{CONTENTS}

\section{Page}

ACKNOWLEDGMENTS

CONTENTS

ii

TABLES $\quad$ iv

FIGURES

CHAPTER

I. OXADIAZINANONE RESEARCH-HOW AND WHY? 1

Fractional Crystallization $\quad 1$

A Brief History of Quasienantiomer Research 3

Terminology and Basic Principles $\quad 5$

Oxadiazinanone-What is in a Name? 9

Why Study Quasiracemates? 11

Previous Quasiracemic Crystal Research 12

$\begin{array}{ll}\text { II. EXPERIMENTAL } & 15\end{array}$

Synthesis of Oxadiazinanones Used in Crystallographic Studies 15

Change in ${ }^{1} \mathrm{H}$ NMR After Nitrosation of Alkyl Derivative 19

Experimental Procedure for the Synthesis of $(5 R, 6 S)-4-C y c l o p e n t y l-5-$ methyl-6-phenyl-2H-1,3,4-oxadiazinan-2-one, 1

Experimental Procedure for the Intended Synthesis of $(5 R, 6 S)-4-$ Ethyl-5-methyl-6-phenyl-2H-1,3,4-oxadiazinan-2-one, $5 \quad 24$

Experimental Procedure for the Intended Synthesis of $(5 R, 6 S)-4-$ n-Butyl-5-methyl-6-phenyl-2H-1,3,4-oxadiazinan-2-one, $6 \quad 27$

Crystallization Procedure $\quad 29$

X-Ray Diffraction 30

III. CRYSTALLOGRAPHIC RESULTS 32

Crystallographic Details of $(5 R, 6 S)-4-C y c l o p e n t y l-5-$ methyl-6-phenyl-2H-1,3,4-oxadiazinan-2-one, 1 
Crystallographic Details of Racemic 4-Cyclopentyl-5methyl-6-phenyl-2H-1,3,4-oxadiazinan-2-one, 2

Crystallographic Details of Quasiracemate of $(5 R, 6 S)-4-$ cyclopentyl-5-methyl-6-phenyl-2H-1,3,4-oxadiazinan2-one and (5S,6R)-4-isopropyl-5-methyl-6-phenyl-2H1,3,4-oxadiazinan-2-one, 3

Crystallographic Details of Quasiracemate of $(5 R, 6 S)$-4-Cyclopentyl5-methyl-6-phenyl-2H-1,3,4-oxadiazinan-2-one and (5S,6R)4-Cyclohexyl-5-methyl-6 phenyl-2H-1,3,4-oxadiazina-2-one, 4

Crystallographic Details of 5

Crystallographic Details of $\mathbf{6}$

Summary of Crystallographic Details of Crystals Studied in

This Research

IV. DISCUSSION OF CRYSTAL STRUCTURES

Summary of the Formation of Quasiracemates and Some Key

Crystallographic Data Pertaining to the Oxadiazinanones Studied

Analysis of $(5 R, 6 S)-4-C y c l o p e n t y l-5-m e t h y l-6-p h e n y l-2 H-1,3,4-$

oxadiazinan-2-one, 1

Analysis of the Racemate of 4-Cyclopentyl-5-methyl-6-phenyl-2H-

1,3,4-oxadiazinan-2-one, 2

68

Analysis of the Quasiracemate of (5R,6S)-5-Methyl-4-cyclopentyl-6phenyl-2H-1,3,4-oxadiazinan-2-one and (5S,6R)-4-Isopropyl5-methyl-6-phenyl-2H-1,3,4-oxadiazinan-2-one, 3

Analysis of the Quasiracemate of (5R,6S)-5-Methyl-4-cyclopentyl-6phenyl-2H-1,3,4-oxadiazinan-2-one and (5S,6R)-4-Cylohexyl5-methyl-6-phenyl-2H-1,3,4-oxadiazinan-2-one, 4

Analysis of 5

General Conclusions and Future Work 


\section{TABLES}

Table

Page

2.1 Aldehydes and ketones used in the synthesis of $\mathrm{N}_{4}$ substituted norephedrine.

3.1 Bond lengths of the central oxadiazinanone rings in the asymmetric unit of 1.

3.2 Bond angles of the central oxadiazinanone rings in the asymmetric unit of $\mathbf{1}$.

3.3 Torsion angles of the central oxadiazinanone rings in the asymmetric unit of $\mathbf{1}$.

3.4 Donor-Acceptor Hydrogen bond lengths in $\mathbf{1}$.

3.5 Bond lengths in the central oxadiazinanone ring of 2.

3.6 Bond angles of the central oxadiazinanone ring of 2 .

3.7 Torsion angles of the central oxadiazinanone ring in the asymmetric unit of 2.

3.8 Donor-Acceptor Hydrogen bond lengths in $\mathbf{2}$.

3.9 Bond lengths of the central oxadiazinanone atoms of $\mathbf{3}$.

3.10 Bond angles of the central oxadiazinanone rings in the asymmetric unit of 3.

3.11 Torsion angles of the central rings in the asymmetric unit of 3.

3.12 Donor-Acceptor Hydrogen bond lengths in 3.

3.13 Bond lengths in the central oxadiazinanone ring of 4.

3.14 Bond angles in the central oxadiazinanone ring of 4 . 
3.15 Torsion angles of the central oxadiazinanone ring of $4 . \quad 46$

3.16 Donor-Acceptor Hydrogen bond lengths in $4 . \quad 46$

3.17 Bond lengths between atoms in the asymmetric unit of 5.

3.18 Bond angles in 5. 50

3.19 Torsion angles in asymmetric unit of 5.

3.20 Donor-Acceptor Hydrogen bond lengths in 5.

3.21 Bond lengths between atoms in the asymmetric unit of 6.

$3.22 \quad$ Bond angles in 6. 54

3.23 Torsion angles in asymmetric unit of 6 . 55

3.24 Donor-Acceptor Hydrogen bond lengths in 6.

3.25 Summary data for crystallographic details of the oxadiazinanone crystals in this thesis. 56

3.26 Summary of crystallographic details of crystals studied in this research which were not oxadiazinanones.

4.1 Fractional crystallization results of the combination of $(5 R, 6 S)$ oxadiazinanones and $(5 S, 6 R)$ oxadiazinanones.

4.2 Crystallographic details of enantiomerically pure compounds. 59

4.3 Crystallographic details of racemic compounds. 60

4.4 Crystallographic details of quasiracemic compounds. 61

4.5 The occurrence frequency of structures between Z' ranges in The Cambridge Structural Database in 2013. ${ }^{39}$

4.6 Torsion angles and bond distances in the hydrogen bond motifs of 2, 3 and 4. 


\section{FIGURES}

Figure $\quad$ Page

1.1 Comparison of (left) chlorosuccinic acid and (right) bromosuccinic acid. 4

1.2 The quasiracemate of $(R)$-2-(3-bromophenoxy)propionic acid (right) and (S)-2-(3-methoxyphenoxy)propionic acid (left).

1.3 On the left is (S)-2-(2-bromo)propionic acid and on the right is $(R)$-2-(2-bromo)propionic acid.

1.4 Quasienantiomers which differ only because one molecule has a Cl atom instead of a $\mathrm{Br}$ atom. The dotted line represents a mirror plane.

1.5 Quasienantiomers which only differ in the presence of a methyl group in one(top) and a tri-isopropyl silyl.ether functional group in the other (bottom).The dotted line represents a mirror plane.

1.6 Line drawing of (5S,6R)-4-cyclopentyl-5-methyl-6-phenyl-2H1,3,4-oxadiazinan-2-one.

1.7 Line drawing of $(5 R, 6 S)-4-R-5-m e t h y l-6-p h e n y l-1,3,4-$ oxadiazinan-2-one.

1.8 Line drawing of (left) (5R,6S)-4-cyclopentyl-5-methyl-6-phenyl-2H1,3,4-oxadiazinan-2-one and (right) (5S,6R)-4-cyclopentyl-5-methyl-6phenyl-2H-1,3,4-oxadiazinan-2-one.

1.9 Line drawings of (left) (5R,6S)-4-isopropryl-5-methyl6-phenyl-2H-1,3,4- oxadiazinan-2-one and (right) (5S,6R)-4cyclohexyl-5-methyl-6-phenyl-2H-1,3,4-oxadiazinan-2-one

2.1 Step 1: Reductive alkylation of $(1 S, 2 R)$ norephedrine.

2.2 Mechanism for the reductive alkylation of an amine. ${ }^{28}$

2.3 Step 2: Nitrosation of the alkylated derivative of $(1 S, 2 R)$ norephedrine.

2.4 Mechanism for the nitrosation of an alkylated norephedrine. ${ }^{28}$ 
2.5 Step 3: Hydrazine-formation from the reduction of the nitrosate of the alkyl derivative of $(1 S, 2 R)$ norephedrine.

2.6 Step 4: Cyclization of the hydrazine forming the 1,3,4 oxadiazinanone.

2.7 The (E)-rotamer (left) and the ( $Z$ )-rotamer (right) of the nitrosamines of the cyclopentyl derivatives of $(1 S, 2 R)$ norephedrine.

2.8 $\quad{ }^{1} \mathrm{H}$ NMR spectrum of the cyclopentyl derivative of $(1 S, 2 R)$ norephedrine.

$2.9{ }^{1} \mathrm{H}$ NMR spectrum of the nitrosate of the cyclopentyl derivative of $(1 S, 2 R)$ norephedrine. Note the presence of the two doublets caused by the two different nitrosate rotamers.

2.10 Line drawing of 5. This is not an oxadiazinanone, but the product of an oxadiazinanone reaction which did not cyclize.

2.11 Line drawing of $\mathbf{6}$. This is not an oxadiazinanone, but, instead is the product of an oxadiazinanone reaction which did not cyclize.

3.1 Line diagram of $\mathbf{1}$.

3.2 Ellipsoid depiction of $\mathbf{1}$.

3.3 Line drawings of the two enantiomers of 2.

3.4 Ellipsoid depiction of asymmetric unit of 2.

3.5 Line drawings of (left) (5R,6S)-4-cyclopentyl-5-methyl-6-phenyl-2H1,3,4-oxadiazinan-2-one and (right) (5S,6R)-4-cyclopentyl-5-methyl -6- phenyl-2H-3,4-oxadiazinan-2-one.

3.6 Ellipsoid depiction of $\mathbf{3}$.

3.7 (5S,6R)-4-Cyclohexyl-5-methyl-6-phenyl-2H-1,3,4oxadiazinan-2-one (left) and (5R,6S) 5-methyl-4-cyclopentyl-6phenyl-2H1,3,4-oxadiazinan-2-one (right).

3.8 Ellipsoid diagram of $\mathbf{4}$.

3.9 Line drawing of 5.

3.10 Ellipsoid depiction of 5. 
$3.11 \quad$ Line drawing of $\mathbf{6}$.

3.12 Ellipsoid depiction of 6.

4.1 Line drawings of $\mathbf{9 , 1 0}$, and $\mathbf{1 2}$. 63

4.2 Packing diagram of $1 . \quad 66$

4.3 Overlay of the two molecules in the asymmetric unit of $\mathbf{1 .} 67$

$4.4 \quad$ Packing diagram of $2 . \quad 68$

4.5 Packing diagram of $3 . \quad 69$

$\begin{array}{lll}4.6 & \text { Overlay of } \mathbf{2} \text { and } 3 . & 70\end{array}$

4.7 The hydrogen-bonded dimers of $\mathbf{2}$ (top) and $\mathbf{3}$ (bottom). 71

4.8 The upper structure is a wireframe depiction of the quasiracemate, $\mathbf{3}$, and the lower structure is that of the racemate, 1. Both structures are shown from a perspective parallel to the hydrogen-bonds. 72

4.9 Packing diagram of the unit cell of $4 . \quad 73$

4.10 Overlay of 2 and $4 . \quad 74$

4.11 Hydrogen-bond comparison of 2 (top) and 4 (bottom). 75

4.12 Asymmetric unit of $\mathbf{4}$ solved in $P 2_{1} /$ c space group 76

4.13 An attempt to cyclize the hydrazine of the ethyl derivative of $(1 S, 2 R)$ norephedrine resulted in $5 . \quad 78$

4.14 Packing diagram of the unit cell of 5. 78

4.15 An attempt to cyclize the hydrazine of the $n$-butyl derivative of $(1 S, 2 R)$ norephedrine resulted in synthesis of $\mathbf{6}$.

4.16 Packing diagram of the unit cell of 6.80 


\section{CHAPTER I}

\section{OXADIAZINANONE RESEARCH-HOW AND WHY?}

\section{Fractional Crystallization}

Typically, when different compounds are mixed together and crystallized, the components of the mixture crystallize as pure compounds. This happens at different times based on the solubility of each of the compounds. This process, called fractional crystallization, is used as a separation technique in industrial applications. In a solution, the desired product is usually present in a greater concentration than the impurities and, generally, is deposited first. The impurities are left in solution. The failure to fractionally crystallize occurs when one of the compounds co-crystallizes with another compound also present in the solution. This results in a new crystalline product which is a combination of the original compounds. ${ }^{1}$

Several conditions seem to increase the likelihood of co-crystallization. When enantiomers crystallize they almost always co-crystalize as the corresponding racemate. This is due to the presence of symmetry elements, such as inversion or glide planes, which are very favorable to crystal-packing as well as the fact that both enantiomers have identical physical properties, including solubility. In fact, the occurrence of fractional crystallization of enantiomers is so rare it is given a special name, spontaneous resolution. When a crystal has inversion or glide planes as symmetry elements, the crystal is able to pack more densely which decreases the size of the crystal. 
This decrease in size and increase in density is favorable to crystal. packing. ${ }^{2}$ Quasiracemates, as defined by the International, Union of Pure and Applied Chemistry (IUPAC), are crystals composed of a 1:1 ratio of "almost enantiomers.”3 Husebye, in the 1960s, reported that quasiracemates generally have structures with a great deal of similarity to the corresponding racemates. This observation may provide some support to the importance of inversion symmetry in co-crystallization. ${ }^{1,4}$ Quasiracemates, because they have pseudo inversion centers, may be able to pack in a smaller volume, thus reducing the size of the asymmetric unit in the same manner as the co-crystallization observed in racemates.

In addition to strong inversion symmetry, hydrogen-bonding contributes to cocrystallization. ${ }^{2}$ Hydrogen bonds are somewhat elastic and this elasticity allows the distances and angles to vary at a small cost in energy which simplifies the bonding in a co-crystal. Therefore, there are a large number of co-crystals with either a carboxylic group or an amide group. ${ }^{1}$

Often, however, it is difficult to determine the reason for co-crystallization. Kelley et al, created a list of quasiracemic crystals. ${ }^{1}$ The definition the researchers employed stated that quasiracemic crystals are co-crystals formed by molecules which are almost enantiomers but differ by the substitution of a few atoms. Of particular note is that the researchers found the formation of quasiracemic crystals is rare. In 2011 there were over 400,000 structures in the Cambridge Structural Database. Of these, only 114 quasiracemates were identified as quasiracemates. $^{1}$ 


\section{A Brief History of Quasienantiomer Research}

Louis Pasteur's earliest chemical studies were in the field of crystallography. In 1846, he found that tartaric acid had optical activity, but paratartaric acid had none leading him to believe there were two kinds of crystals present although most other chemical properties were the same in the two compounds. He noticed that the edges of the crystals were asymmetrical—-the surfaces on some of them were facing right and some, left. Using a dissecting needle and a microscope, he separated the crystals into two piles based on these differences. He recognized the difference in optical activity, extended his observations to the molecular level and realized that the two kinds of crystals were mirror images. He wrote his doctoral dissertation on this subject in $1847 . .^{5,6}$

The basis for chirality and stereoisomerism was laid by van’t Hoff and Le Bel in 1874 when, independently, they each proposed the existence of tetrahedral carbon with a tetrahedral valence. In consequence of this, the idea of enantiomerism came into being. Enantiomers are compounds with the same chemical constitution which are mirrorimages of each other, but are non-superimposable. ${ }^{7}$ William Thomson, (Lord Kelvin), first introduced this definition in 1883 at a lecture given to the Oxford University Junior Scientific Club. ${ }^{8}$ It was not reintroduced into stereochemical literature until 1965 by Mislow $^{9}$ and 1966 Cahn, Ingold and Prelog. ${ }^{5}$

In 1899, Centnerswer studied a 1:1 mixture of (-)-bromosuccinic acid (Figure 1.1 left) and (+)-chlorosuccinic acid Figure 1.1 right). ${ }^{7,}{ }^{10}$ He found that this mixture had phase properties similar to those of the corresponding true racemic compounds for each of the molecules. The mixture contained neither isomers nor enantiomers, but had an “almost” or “pseudo” mirror-image relationship., 10 

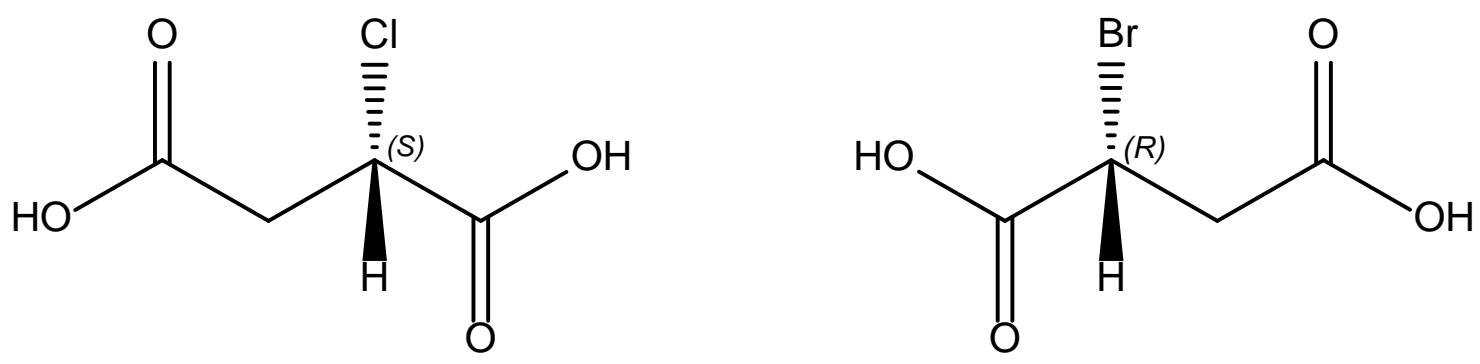

Figure 1.1 Comparison of (left) chlorosuccinic acid and (right) bromosuccinic acid.

Most of the studies of quasiracemates, and the reasons for their formation, were conducted in the 1960s and 1970s by A. Fredga. ${ }^{11}$ Fredga, in 1960, first used the term "quasi-racemate" to distinguish this class of compounds. ${ }^{12}$ This research used melting point phase diagrams and found that these sufficed to determine true racemic compounds, racemic conglomerates, as well as quasiracemates. A racemic conglomerate is a mixture of two types of crystals where each crystal is composed of only one enantiomer, but the entire mixture has an equimolar amount of both enantiomers. ${ }^{3}$ This study provided clear evidence for the presence of quasiracemates, identified necessary criteria for the selection of sterically similar molecules, and used the molecular topology to design new quasiracemates. ${ }^{11}$

In 1966, Jerome and Isabella Karle determined the structure of the quasiracemates, (R)-2-(3-bromophenoxy) propionic acid and (S)-2-(3-methoxyphenoxy) propionic acid (Figure 1.2) ${ }^{13}$ Their study of the pseudo inversion symmetry of this quasiracemate proved to be one of the first investigations into packing motifs in quasiracemates. The relationship of inversion packing to packing motif is now firmly established. ${ }^{12}$ 


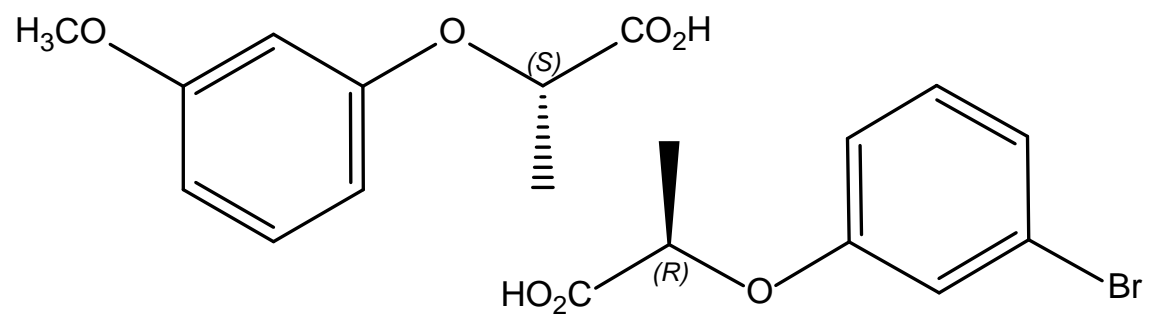

Figure 1.2 The quasiracemate of (R)-2-(3-bromophenoxy)propionic acid (right) and (S)2-(3-methoxyphenoxy)propionic acid (left).

In the 1990's the late Margaret Etter, a pioneer in crystal engineering, studied hydrogen bonding motifs in crystals. The research gave insight into the role of hydrogenbonding in controlling the structures of crystals. The use of graph-sets in describing these motifs has been useful in understanding crystal structure and comparing and contrasting sets of molecules. The terminology proposed by Etter et al., 1990 has been in use for the past twenty years. ${ }^{14}$

In 2002, Fomulu et al. proposed several considerations for the design and synthesis of quasiracemates using Fredga's quasiracemates as a starting point. ${ }^{11}$ These considerations include the fact that the two quasienantiomers need to be isosteric, unique in chemical composition, and opposite in handedness. In addition, it is helpful to have a high degree of similarity between the two components with the presence of hydrogen donor and acceptor groups. ${ }^{11}$ These factors are relevant to our study of quasienantiomers of oxadiazinanones.

\section{Terminology and Basic Principles}

Knowledge of basic terminology and principles are important to the understanding of this research; therefore, it is worthwhile to include a review. 
Enantiomers are compounds which have the same chemical formulas and the same constitutions, but have different three-dimensional structures which are related by a mirror plane as noted in Figure 1.3.
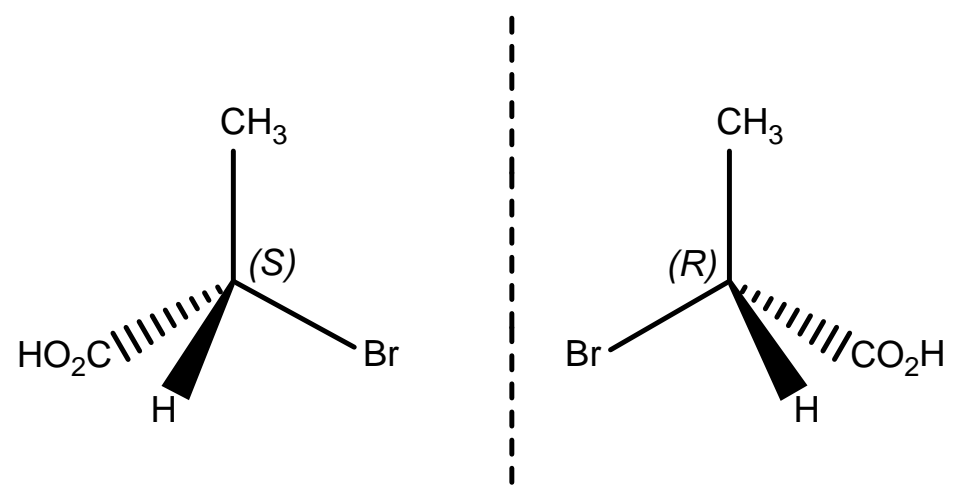

Figure 1.3 On the left is (S)-2-(2-bromo)propionic acid and on the right is $(R)-2-(2-$ bromo)propionic acid.

The IUPAC definition for a racemate is a composite of equimolar quantities of both enantiomers of a compound. ${ }^{3,7}$ Because of the equimolarity of the enantiomers, a racemic mixture will not have optical activity as noted by Pasteur. ${ }^{6}$

A quasiracemate is a compound composed of two "almost" or similar enantiomers. There is some ambiguity in the definition due to the use of the word, "similar.” In an article by Fumulo et al., the definition is worded as "a molecular crystal derived from a true racemate by a not-too-extensive change in the structure of one of the two enantiomeric components." ${ }^{, 11}$ How similar do the enantiomers need to be? The two molecules in Figure 1.4 are almost alike differing only by the $\mathrm{Br}$ or $\mathrm{Cl}$ atom. The bromine in the structure on the left has been replaced by chlorine in the structure on the right, as 
indicated by the arrows. The two molecules in Figure 1.5 also differ at only one point, but the difference involves many more atoms.
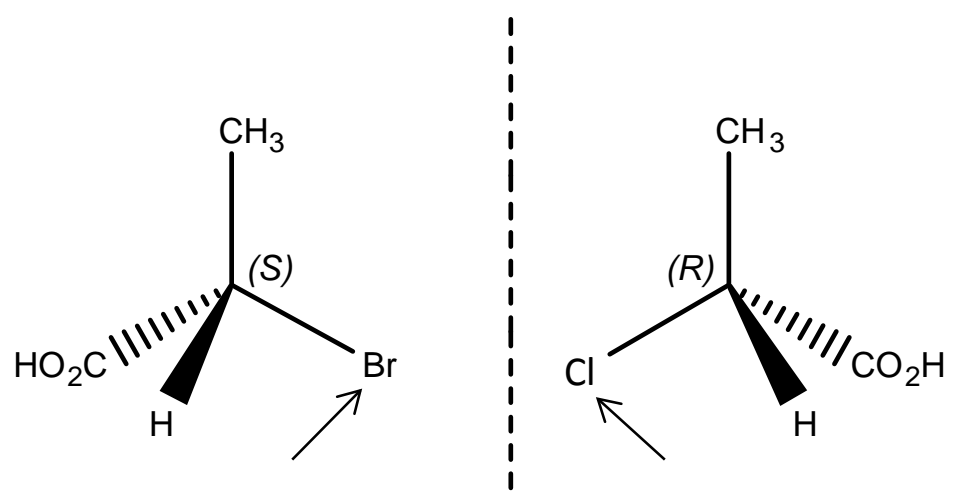

Figure 1.4 Quasienantiomers which differ only because one molecule has a $\mathrm{Cl}$ atom instead of a $\mathrm{Br}$ atom. The dotted line represents a mirror plane.<smiles>Cc1ccc(S(=O)(=O)OC[C@H]2CN(c3ccccc3)C(=O)O2)cc1</smiles>

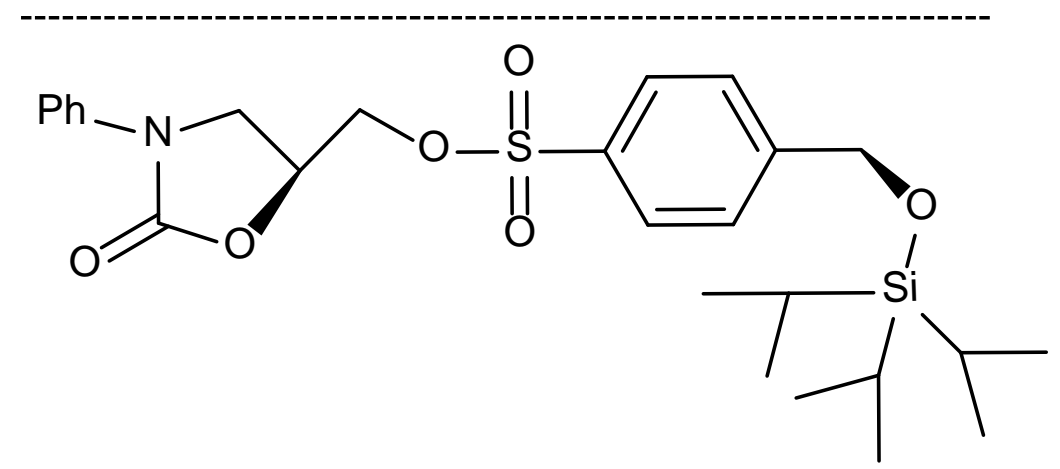

Figure 1.5 Quasienantiomers which only differ in the presence of a methyl group in one(top) and a tri-isopropyl silyl ether functional group in the other (bottom). The dotted line represents a mirror plane. ${ }^{7}$

Comparing Figures 1.4 and 1.5, one can see there is a much greater difference between the quasienantiomers in Figure 1.5 than those in Figure 1.4. The methyl (Me) 
group in the structure on top in Figure 1.5 has been exchanged for a tri-isopropyl silyl ether group in the bottom structure. Although all of the other atoms in the structures are identical in identity and location, the molecules seem to be quite different.

It is difficult, therefore, to define quasiracemate in a very specific manner. To crystallographers, the definition most generally refers to a crystal comprised of compounds that are very nearly enantiomers with differences of a small number of atoms. This is a very "loose" definition as the "small number" can vary greatly. To synthetic chemists, the difference in the quasienantiomers could be a small such as the difference in a methyl and an isopropyl group or, at the other extreme, the difference could be very large like the difference between a methyl and a polystyrene group. In the present research, a general supposition has been made that the quasienantiomers are closely related and able to form a co-crystal.

Crystallography has a unique vocabulary and a short review may prove to be helpful. Crystals are an arrangement of atoms repeating in a three-dimensional pattern. ${ }^{15}$ The arrangement can go infinitely in all directions by repeating the basic unit of the crystal. This basic unit is called the unit cell. Each unit cell has the same atomic contents arranged in the same way. The atoms within the unit cell can be further reduced to an asymmetric unit. An asymmetric unit is the smallest unit of volume that contains all of the structural information about the crystal. By applying symmetry operations to the asymmetric unit, such as inversion, translation, reflection, etc., the unit cell can be reproduced. The number of molecules in the asymmetric unit is designated by $Z$ ' and the number of molecules in the unit cell is designated as $Z{ }^{15}$ 


\section{Oxadiazinanone-What is in a Name?}

The typical nomenclature of the oxadiazinanones can be confusing as one looks over the literature of the last thirteen years. This is evidenced by looking at the titles of papers authored or co-authored by Dr. G.M. Ferrence. In 2001, research involving 3,4,5,6-tetrahydro-2-H-1,3,4-oxadiazin-2-one was reported. ${ }^{16}$ In articles published in 2002, research was also published reporting $\mathrm{N}_{3}$-substituted [1,3,4]-oxadiazinan-2-ones. ${ }^{17}$ In 2004, one finds research on a group of compounds called oxadiazinones. ${ }^{18}$ In 2008, two papers were written on research involving chirality and substituted 1,3,4oxadiazinan-2-ones. ${ }^{19 a, 19 b}$ In 2009 a paper was published on (5S,6R)-4-isopropyl-5methyl-6-phenyl-3-propanoyl-2H-1,3,4-oxadiazinan-2-one. ${ }^{20}$ A slight variation is found in 2009 when the compound is a thione instead of a ketone. In this case the nomenclature changes to (5S,6R)-5-methyl-6-phenyl-4-propyl-1,3,4-oxadiazinane-2-thione. ${ }^{21}$ Why the differences? What do the different names mean?

IUPAC recommends using the Hantzsch-Widman nomenclature in the naming of heterocycles. ${ }^{22}$ The rings are named by combining prefixes with a stem. In the case of oxadiazinanones, the prefix 'oxa' refers to the oxygen atoms in the ring and the 'az' to the nitrogen atoms in the ring; the 'di' reflects the fact there are two nitrogen atoms. The order of the names of the heteroatoms is dictated by IUPAC as $\mathrm{O}, \mathrm{N}$, and, then, $\mathrm{S}$. Other atom preferences are listed in the literature. ${ }^{23}$ Typically the 'az' would be 'aza,' but phonetics dictates the omission of the final 'a.' This type of omission is called eliding. The stem for a saturated 6-member heterocycle with nitrogen and oxygen in the ring is 'inane' but in the case of these compounds the final 'e' is omitted, again, for phonetic reasons. In some cases the stem 'inane' is changed to 'ine' and the saturated atoms that 
are present in the compound are denoted by using terms such as 'tetrahydro,' or 'dihydro.' Because oxadiazinanones are ketones, the name of the compound ends in 'one.' The numbering in heterocyclic rings becomes necessary when substituents are placed on the ring. The numbering begins with a hetero-atom, and then the substituents are numbered so that the lowest combination of numbers is assigned. The substituents are placed in alphabetical order. The positions of the substituents play no role in the order of the numbering. The substituents are included in alphabetical order preceded by the number of the atom to which they are attached. ${ }^{23}$ In addition to these rules, unsaturated atoms located in the ring are designated by using ' $H$.' This reflects that the parent hydride would have hydrogen atoms in this position.

Using the compound in Figure 1.6 as an example, the proper nomenclature will be discussed. In this heterocycle the chirality is described by $(5 S, 6 R)$.

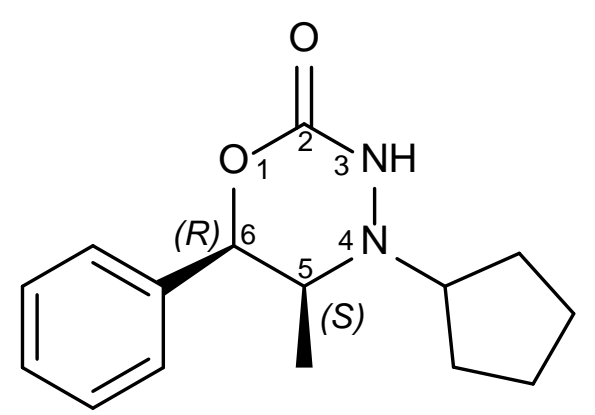

Figure 1.6 Line drawing of (5S,6R)-4-cyclopentyl-5-methyl-6-phenyl-2H-1,3,4oxadiazinan-2-one.

The substituents are listed in alphabetical order with the number of the atom to which they are attached preceding the name of the substituent. The order of the atom numbers is chosen by starting with the oxygen atom as atom 1 and then numbering the rest. This 
numbering scheme results in the lowest combination of position-numbers of heteroatoms as possible. The $2 \mathrm{H}$ indicates an atom where the parent hydride would have hydrogen atoms affixed, but it does not in this molecule because of the carbonyl group. The "1,3,4oxadiaz" portion of the name refers to the oxygen atom and two nitrogen atoms in the heterocycle. The substituents are arranged in alphabetical order preceded by the atom number to which they are attached. The prefix, "inan," refers to a saturated heterocycle containing nitrogen. The stem, "2-one," refers to the fact that this is a ketone with the carbonyl on atom 2.

\section{Why Study Quasiracemates?}

Some reasons for the study of quasienantiomers given in a review by Zhang and Curran are that they can be used as tools for enantiomer identification, enantiomer analysis, separation of chemical components and enantiomer synthesis. ${ }^{7}$ Although all of these pragmatic uses are valuable, the current study is more esoterically focused on how and why oxadiazinanones form quasiracemates. This is related to the discussion of Fomolu and co-workers about the study of quasiracemates in the field of supramolecular chemistry. Using comparisons of quasiracemate, enantiomer, and racemate crystals, the molecular architecture can be studied. ${ }^{11}$ The database of quasiracemic crystals needs to be as large as possible in order to determine general principles which cause the crystallization of quasiracemic crystals.

Additionally, oxadiazinanones have been synthesized fairly easily using methods developed by Hitchcock and his group. ${ }^{24,25}$ Decent yields have been reported. The compounds are easily purified with flash chromatography. Optically pure norephedrine or ephedrine starting materials result in optically pure substituted oxadiazinanones. 


\section{Previous Quasiracemic Crystal Research}

The research group of Dr. Shawn Hitchcock at Illinois State University has synthesized and studied a variety of oxadiazinanones as a route to forming chiral auxiliaries for pharmaceutical studies. ${ }^{25}$ In an effort to understand some principles of crystal structure and chirality, Kate Edler used these immediately-available oxadiazinanones in her research. ${ }^{26}$ In the course of the research, serendipitously, she mixed together norephedrine-based (5S,6R)-4-isopropryl-5-methyl-6-phenyl-2H-1,3,4oxadiazinan-2-one and (5R,6S)-4-cyclohexyl-5-methyl-6-phenyl-2H-1,3,4-oxadiazinan2-one in approximately equal molarities. These crystals resulted in the first of the quasiracemic oxadiazinanone crystals studied by the Ferrence group. ${ }^{26}$

Ignoring epimers, there are two oxadiazinanone enantiomers for each norephedrine derived N-4-substituted variant. Figure 1.7 shows a line drawing of (5R,6S)-6-phenyl-4-R-(1,3,4)-oxadiazin-2-one. The arrow points to the nitrogen with the substituted variant.

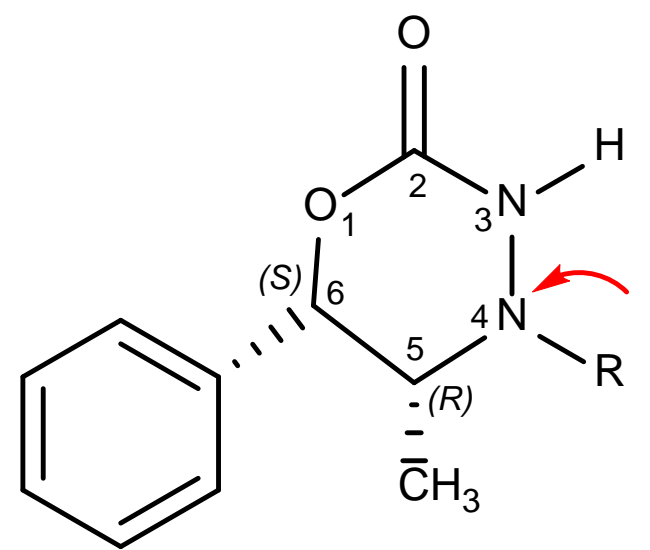

Figure 1.7 Line drawing of (5R,6S)-4-R-5-methyl-6-phenyl-1,3,4-oxadiazinan-2-one. 
For example, using the cyclopentyl functional group, the two oxadiazinanone structures in Figure 1.8, when crystallized, form a racemic crystal.
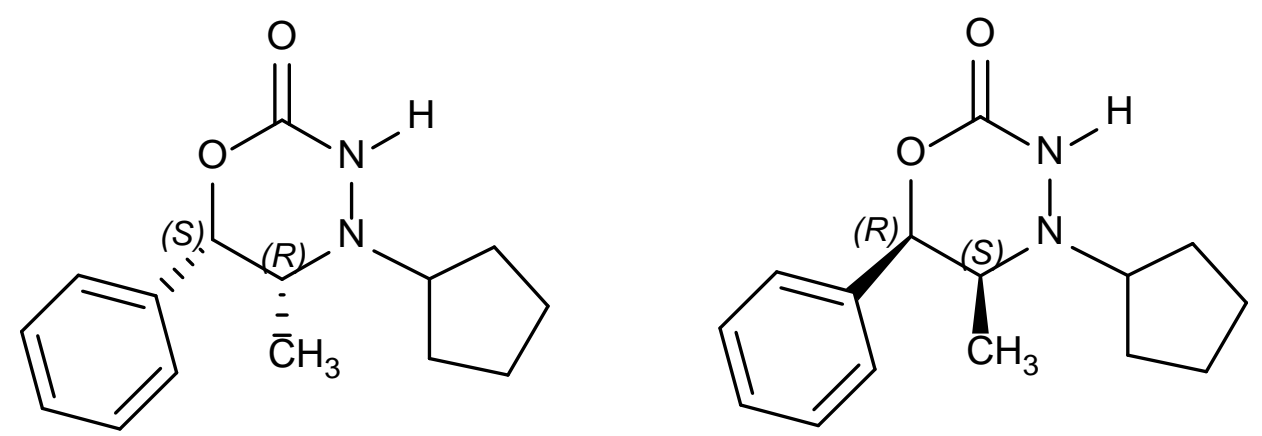

Figure 1.8 Line drawing of (left) (5R,6S)-4-cyclopentyl-5-methyl-6-phenyl-2H-1,3,4oxadiazinan-2-one and (right) (5S,6R)-4-cyclopentyl-5-methyl-6-phenyl-2H-1,3,4oxadiazinan-2-one.

If an isopropyl group is substituted for the cyclopentyl group (structure on the left in Figure 1.8) and a cyclohexyl group for the cyclopentyl group (structure on the right), the resulting compounds are shown in Figure 1.9. These compounds cocrystallize forming a quasiracemic crystal.<smiles>CC(C)N1NC(=O)OC(=C2C=C=CC=C2)[C@@H]1C</smiles><smiles>C[C@H]1[C@@H](c2ccccc2)OC(=O)NN1C1CCCCC1</smiles>

Figure 1.9 Line drawings of (left) $(5 R, 6 S)$-4-isopropryl-5-methyl-6-phenyl-2H-1,3,4oxadiazinan-2-one and (right) (5S,6R)-4-cyclohexyl-5-methyl-6-phenyl-2H-1,3,4oxadiazinan-2-one. 
The fact that the oxadiazinanones in Figure 1.9 formed quasiracemic crystals begs the question: would other oxadiazinanones do so as well? To further explore the possibility, it was necessary to acquire a number of oxadiazinanones which varied at the $\mathrm{N}_{4}$ position in both $(5 S, 6 R)$ and $(5 R, 6 S)$ configurations.

The synthesis of these compounds uses either ephedrine (for methyl-substitution) or norephedrine as a starting reactant. ${ }^{27}$ The resulting oxadiazinanone quasienantiomers are then mixed and crystallized in an attempt to form the corresponding quasiracemate. Finally, the crystal structures are studied in an attempt to determine what causes some oxadiazinanone quasienantiomers to cocrystallize and others to fractionally crystallize. 


\section{CHAPTER II}

\section{EXPERIMENTAL}

\section{Synthesis of Oxadiazinanones Used in Crystallographic Studies}

All oxadiazinanone compounds used in this research were either acquired from the lab of Dr. Shawn Hitchcock or synthesized using procedures, or adaptations thereof, devised by the Hitchcock group. ${ }^{27}$ The compound, (5R,6S)-4-cyclopentyl-5-methyl-6phenyl-2H-1,3,4-oxadiazinan-2-one, and its precursors are newly-synthesized products. The synthesis of the ethyl derivative of the oxadiazinanone and the $n$-butyl derivative of the oxadiazinanone, instead of producing the desired oxadiazinanones, resulted in newlysynthesized noncyclized compounds. In addition to these newly-synthesized compounds, two new quasiracemates were formed when (5R,6S)-4-cyclopentyl-5-methyl-6-phenyl2H-1,3,4-oxadiazinan-2-one was crystallized with (5S,6R)-4-cyclohexyl-5-methyl-6phenyl-2H-1,3,4-oxadiazinan-2-one and separately with (5S,6R)-4-isopropyl-5-methyl-6phenyl-2H-1,3,4-oxadiazinan-2-one.

The synthesis of oxadiazinanones includes four main steps: alkylation, nitrosation, hydrazine-formation, and cyclization. Figures 2.1, 2.3, 2.5, and 2.6 show the steps in the reaction using the cyclopentane derivative as an example: Figure 2.2 gives the mechanism for the reductive alkylation. Table 2.1 shows which alkyl groups were added to $\mathrm{N}_{4}$ as a substituent. 


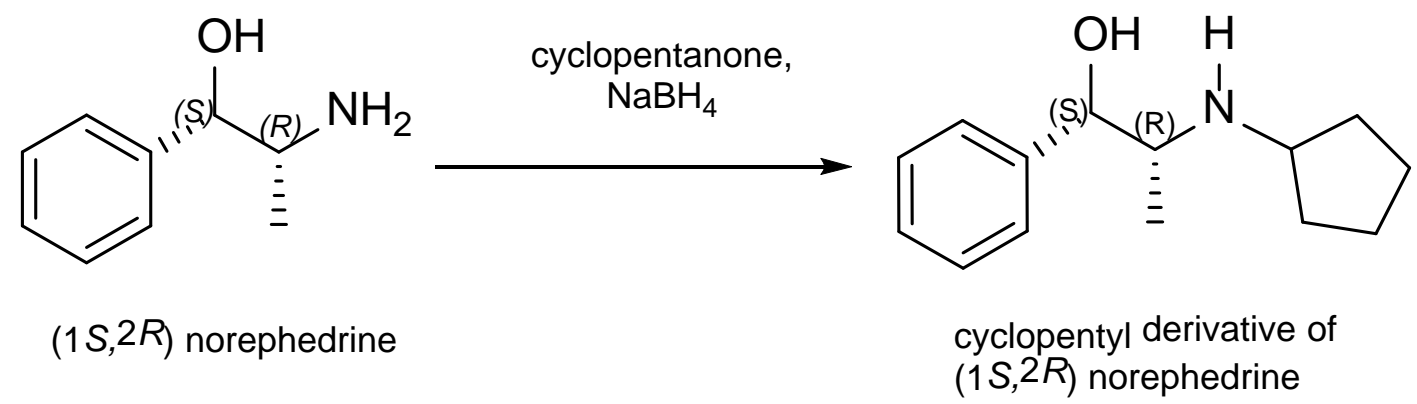

Figure 2.1 Step 1: Reductive alkylation of $(1 S, 2 R)$ norephedrine.

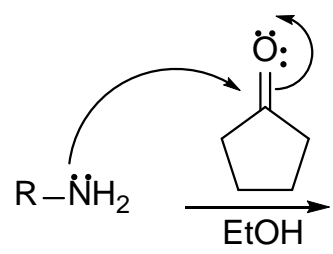

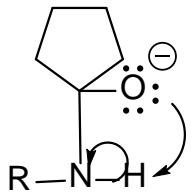

$\oplus$ 'H

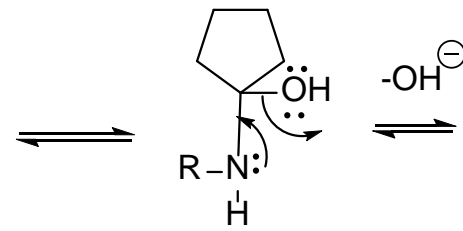

iminium ion<smiles>[R]C1CCCC1</smiles>

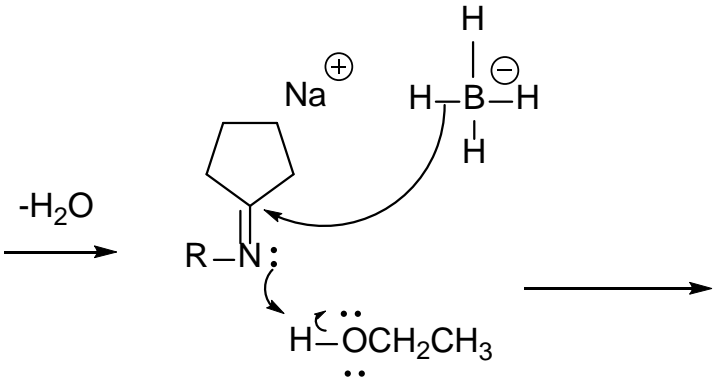<smiles>[R]NC1CCCC1</smiles>

Figure 2.2 Mechanism for the reductive alkylation of an amine. ${ }^{28}$

Table 2.1 Aldehydes and ketones used in the synthesis of $\mathrm{N}_{4}$ substituted norephedrine.

\begin{tabular}{ll}
\hline \multicolumn{1}{c}{$\mathbf{N}_{\mathbf{4}}$ Substituent } & \multicolumn{1}{c}{ Reactant } \\
\hline ethyl & acetaldehyde \\
$n$-propyl & propanal \\
isopropyl & acetone \\
$n$-butyl & butanal \\
cyclohexyl & cyclohexanone \\
cyclopentyl & cyclopentanone \\
\hline
\end{tabular}


Figure 2.3 shows the reaction for the nitrosation of the alkylated norephedrine and the mechanism for the reaction is shown in Figure 2.4.

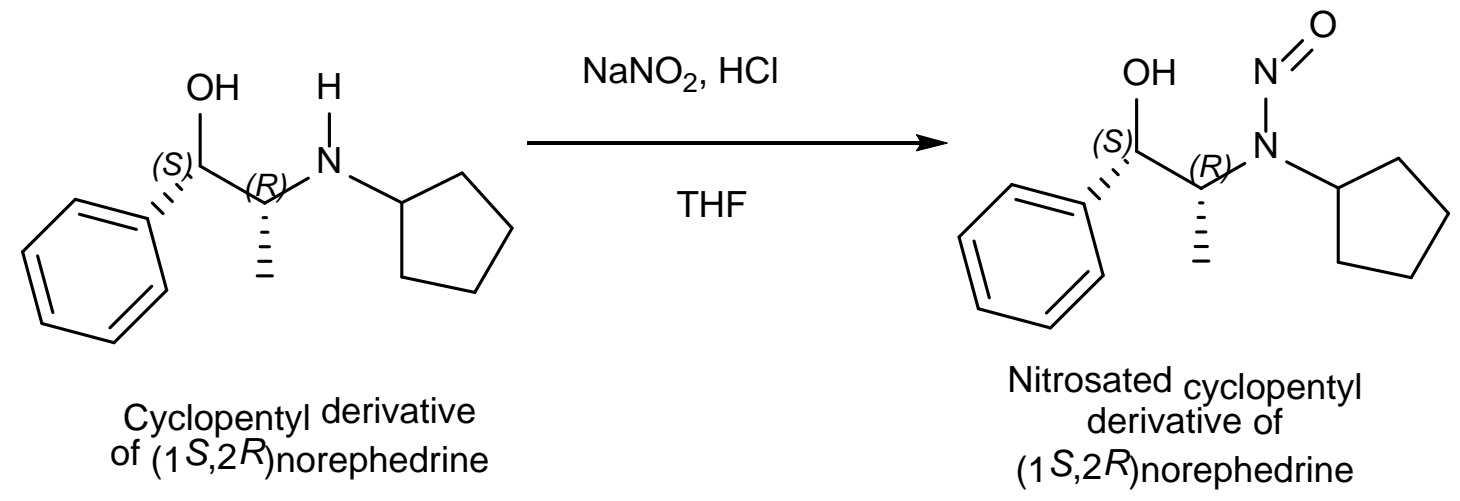

Figure 2.3 Step 2: Nitrosation of the alkylated derivative of $(1 S, 2 R)$ norephedrine.

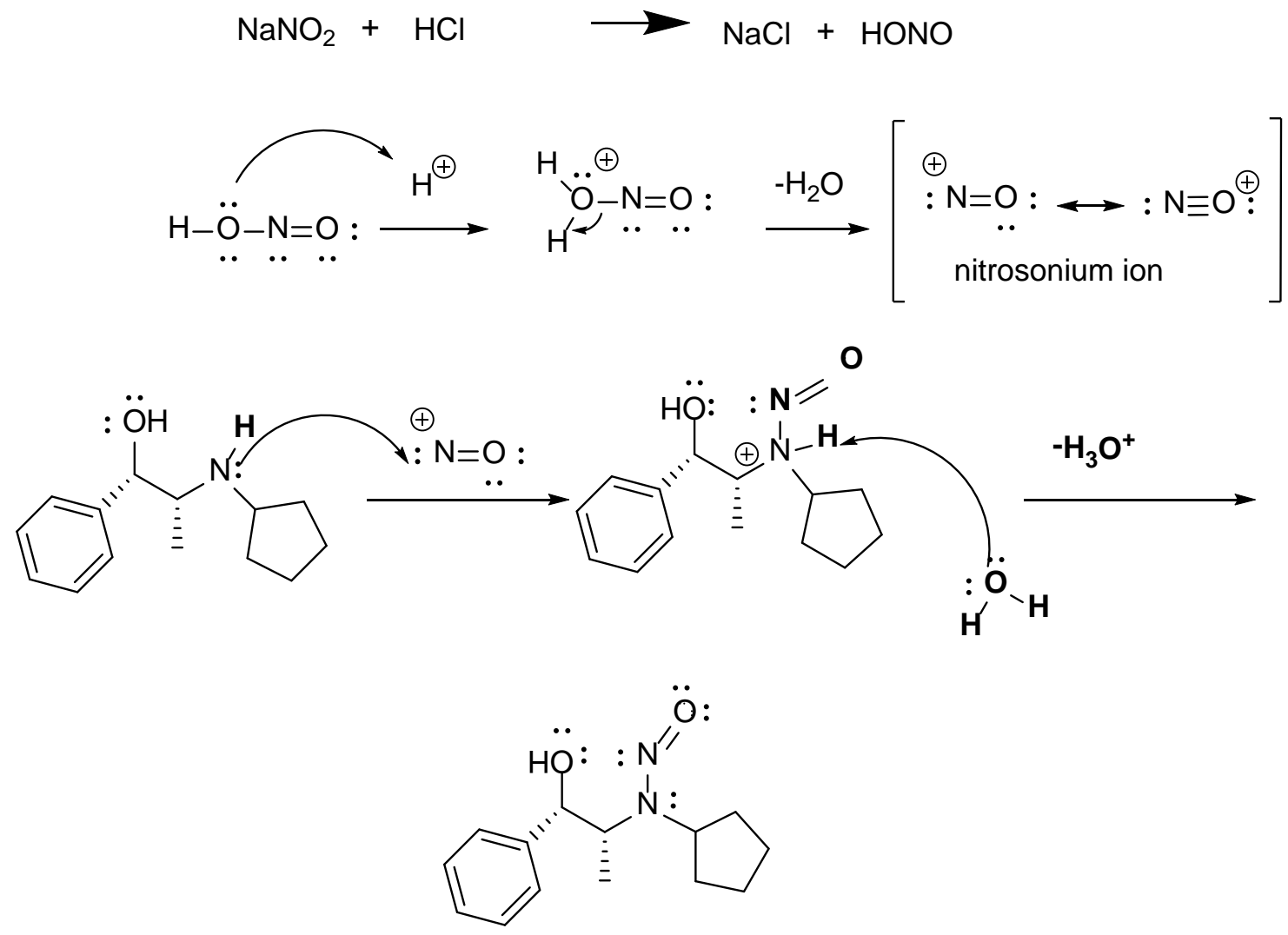

nitrosated alkylated derivative of norephedrine

Figure 2.4 Mechanism for the nitrosation of an alkylated norephedrine. ${ }^{28}$ 
The nitrosate of the alkyl derivative of the norephedrine is part of a group of compounds known to be carcinogenic, so great care must be used to avoid contact with the compound during the synthesis. ${ }^{29}$

At the conclusion of Steps 1 and 2, ${ }^{1} \mathrm{H}$ nuclear magnetic resonance spectroscopy $\left({ }^{1} \mathrm{H}\right.$ NMR) analysis is used to ascertain that the proper product is formed in sufficient purity to continue the synthesis. When the product is not what is expected or is in low yield, typically, the step can be repeated to obtain the desired product and the synthesis can continue. When purification of the product is needed, flash chromatography is utilized.

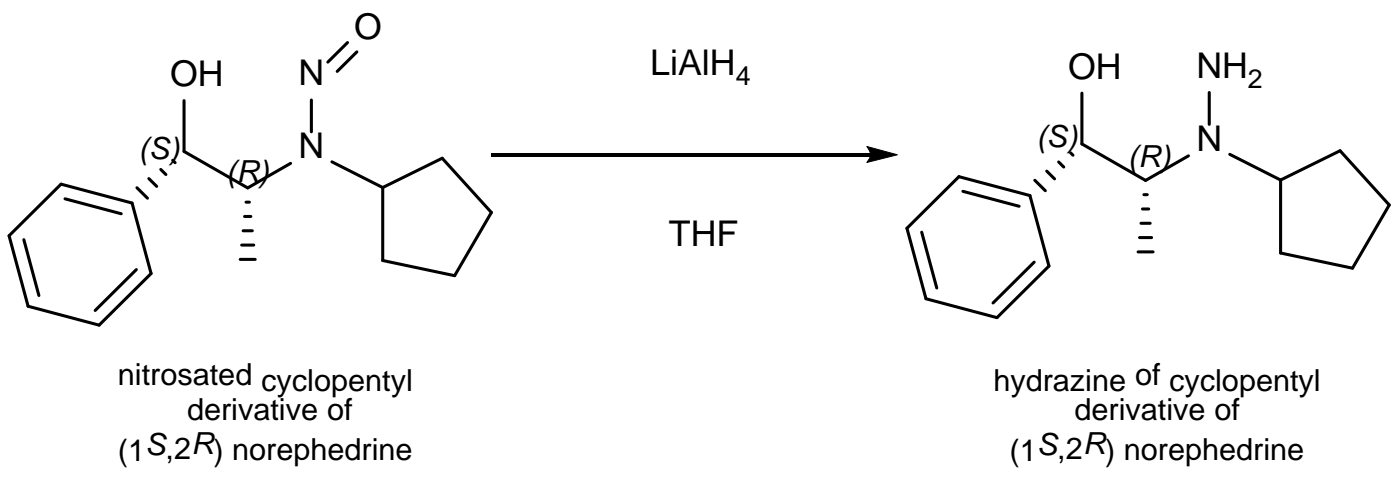

Figure 2.5 Step 3: Hydrazine-formation from the reduction of the nitrosate of the alkyl derivative of $(1 S, 2 R)$ norephedrine. 


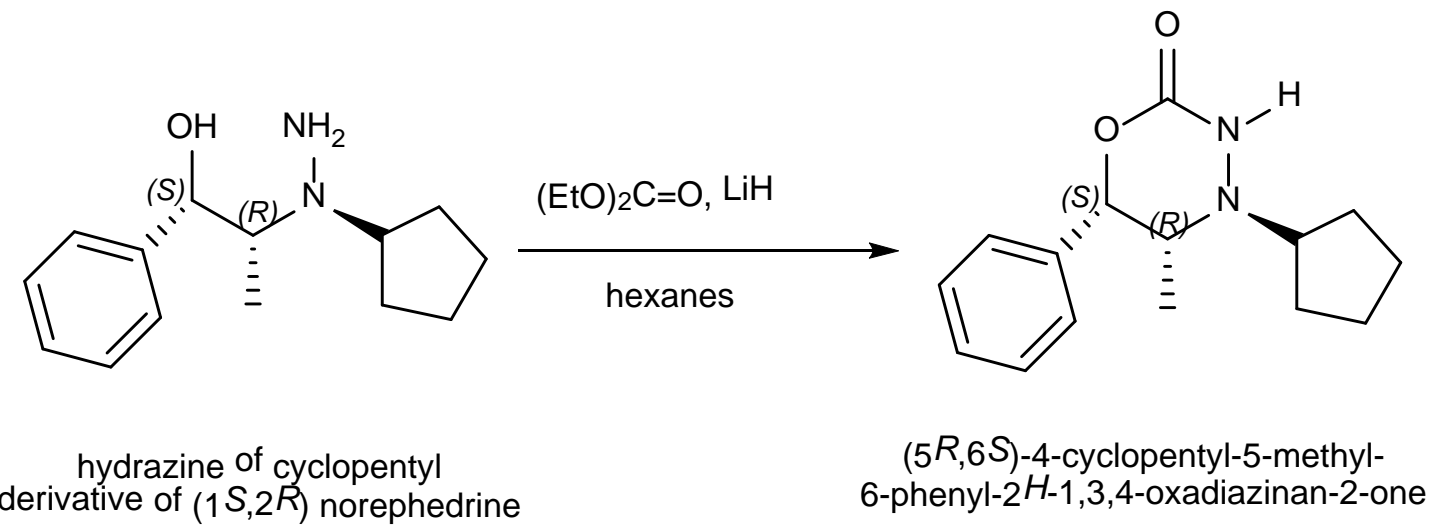

Figure 2.6 Step 4: Cyclization of the hydrazine forming the 1,3,4 oxadiazinanone.

Steps 3 and 4 are completed with as little delay between them as possible because the hydrazine is not stable. ${ }^{1} \mathrm{H}$ NMR testing is typically not done after Step 3 as the hydrazine cannot be purified at this point and remain stable, so the synthesis must continue. Bentley, in his thesis, proposed mechanisms for the third and fourth steps of the procedure; these will not be further explored here. ${ }^{28}$

\section{Change in ${ }^{1} \mathrm{H}$ NMR After Nitrosation of Alkyl Derivative}

The rotamer diastereomers for the nitrosate are shown in Figure 2.7. The rotamer on the right results in having a doublet at $0.8 \mathrm{ppm}$ in the ${ }^{1} \mathrm{H}$ NMR spectrum. The rotamer on the left results in a second doublet further downfield at 1.6 due to the shielding of the oxygen atom in the nitrosate. According to Hitchcock, Casper et al. ${ }^{29}$ the two rotamers exist because the resonance of the $\mathrm{N}=\mathrm{O}$ bond in the structure creates a barrier to rotation of the $\mathrm{N}-\mathrm{N}=\mathrm{O}$ moiety. The $\mathrm{N}-\mathrm{N}$ bond does not rotate until very high temperatures are obtained so both rotamer diastereomers occur simultaneously in the nitrosate of the alkylated derivative of norephedrine. These two rotamers help to verify the presence of the nitrosate. 
The ${ }^{1} \mathrm{H}$ NMR spectra of the alkylated derivative, as seen in Figure 2.8, has a doublet at $0.8 \mathrm{ppm}$ indicating the presence of the methyl group. However, when the $\mathrm{N}$ nitroso amine is formed in the second step of the synthesis, the ${ }^{1} \mathrm{H}$ NMR spectra in Figure 2.9 shows two doublets for the methyl group—one doublet at $0.83 \mathrm{ppm}$ and one downfield at 1.6`. This results from the formation of two diastereomeric rotamers (Figure 2.7) which exist as major and minor species in the product.
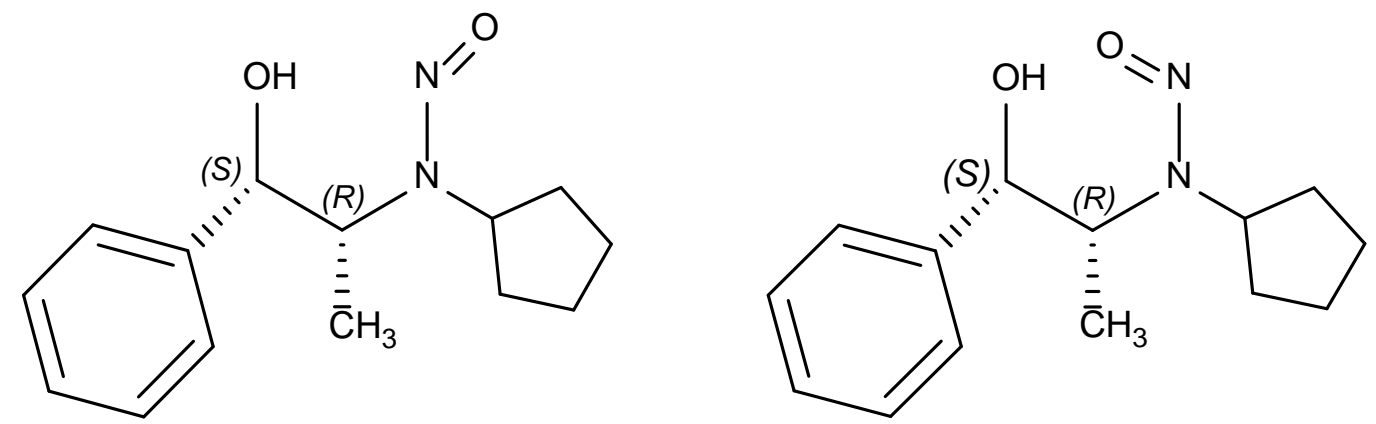

Figure 2.7 The (E)-rotamer (left) and the (Z)-rotamer (right) of the nitrosamines of the cyclopentyl derivatives of $(1 S, 2 R)$ norephedrine. 


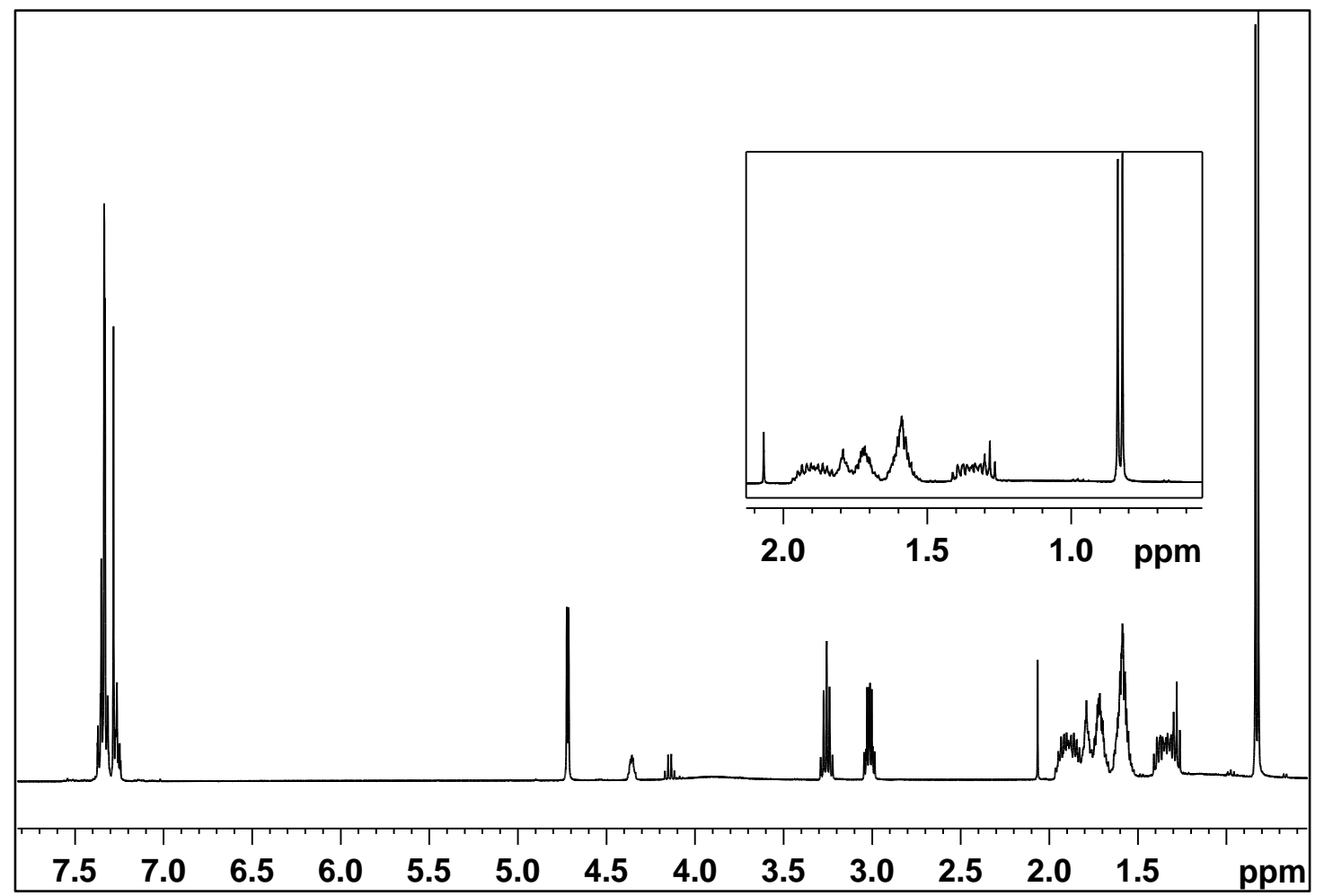

Figure 2.8 ${ }^{1} \mathrm{H}$ NMR spectrum of the cyclopentyl derivative of $(1 S, 2 R)$ norephedrine. 


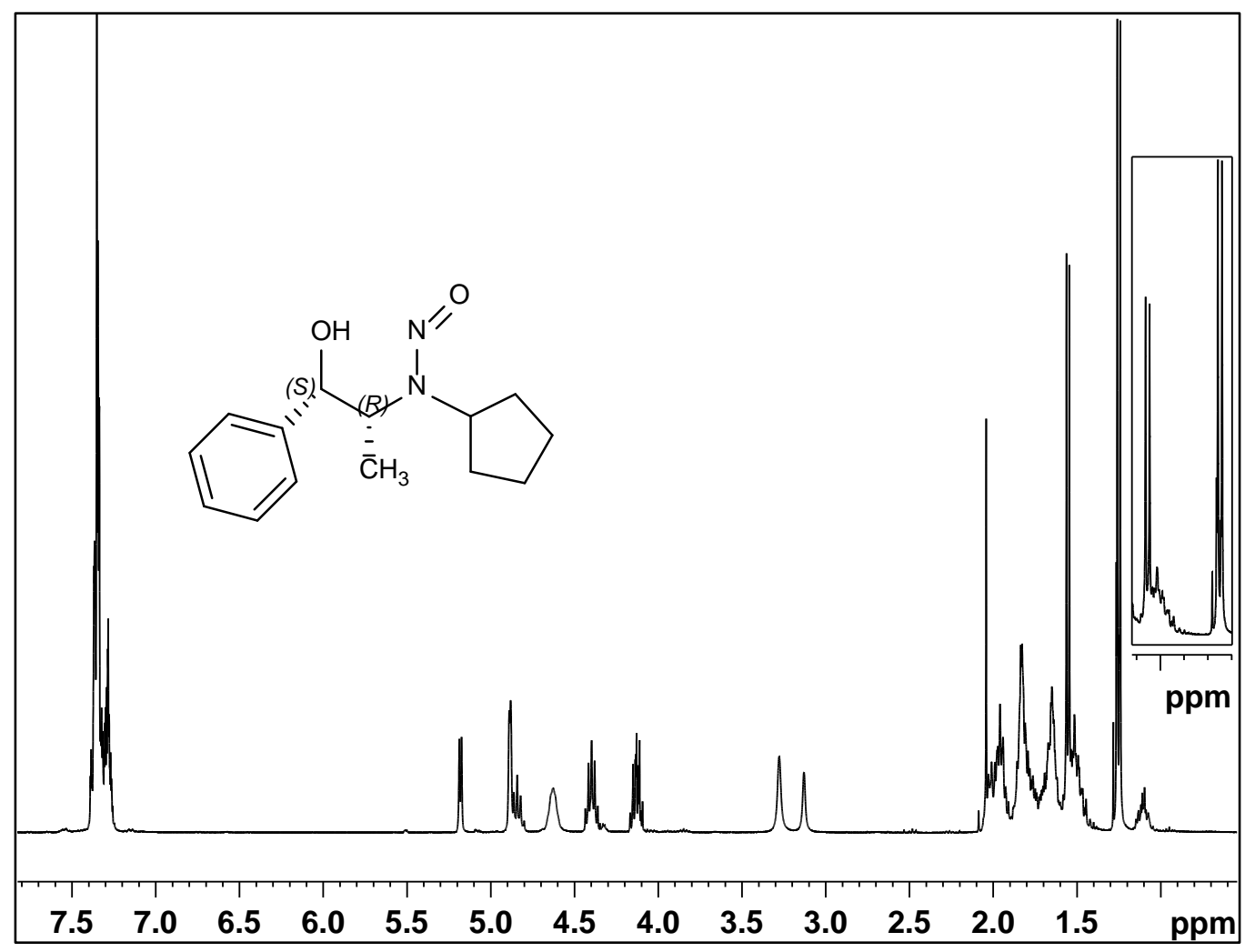

Figure 2.9 ${ }^{1} \mathrm{H}$ NMR spectrum of the nitrosate of the cyclopentyl derivative of $(1 S, 2 R)$ norephedrine. Note the presence of the two doublets caused by the two different nitrosate rotamers.

\section{Experimental Procedure for the Synthesis of $(5 R, 6 S)-4-C y c l o p e n t y l-5-m e t h y l-6-$ phenyl-2H-1,3,4-oxadiazinan-2-one, 1}

Alkylation: (1S,2R) norephedrine (10.00 g, $66.13 \mathrm{mmol})$ and cyclopentanone (7.00 g, $83.2 \mathrm{mmol}$ ) were added to a nitrogen-purged, $1 \mathrm{~L}$ round-bottom flask with ethanol (100 $\mathrm{mL})$. The mixture was refluxed for 24 hours, cooled in an ice bath and $\mathrm{NaBH}_{4}(6.26 \mathrm{~g}$, $165 \mathrm{mmol}$ ) was added over a period of approximately 30 minutes. The flask was removed from the ice and ethanol was added $(100 \mathrm{~mL})$. After the mixture was stirred for an additional 2.5 hours, 1M NaOH (100 mL, $100 \mathrm{mmol})$ was added. Ethanol was removed from the solution using rotary evaporation, and the product was extracted with ethyl acetate, washed with brine, and dried over sodium sulfate resulting in the cyclopentyl 
derivative of $(1 S, 2 R)$ norephedrine (13.73 g, $76.24 \mathrm{mmol}) .{ }^{1} \mathrm{H}$ NMR spectroscopy was used to ascertain the purity of the sample, and it was determined that the product was not fully alkylated. The product was then refluxed overnight under the same conditions and with the same reagents as described previously resulting in the desired product. Nitrosation: The cyclopentyl derivative of $(1 S, 2 R)$ norephedrine was nitrosated by adding $3 \mathrm{M} \mathrm{HCl}(25 \mathrm{~mL}, 75 \mathrm{mmol}$ ) to the alkylated norephedrine. Approximately $60 \mathrm{~mL}$ of tetrahydrofuran (THF) was added, and the mixture was stirred. To the mixture $\mathrm{NaNO}_{2}$ (5.5 g, $79 \mathrm{mmol}$ ) was added. The solution turned pink. The reaction was allowed to proceed for 4 days. Sodium bicarbonate (saturated solution) was added to reach a $\mathrm{pH}$ of 8. THF was removed by rotary evaporation. The final product was extracted with ethyl acetate, washed with brine and dried over $\mathrm{Na}_{2} \mathrm{SO}_{4}$. The ethyl acetate was removed by rotary evaporation resulting in the nitrosated cyclopentyl derivative of $(1 S, 2 R)$ norephedrine (8.19 g, $33.0 \mathrm{mmol})$. One gram was removed for further research and the rest of the product was reduced.

Reduction: Hexanes (200 mL) were added to a flame-dried, nitrogen-purged, 2 L roundbottom flask. Lithium aluminum hydride ( $2.29 \mathrm{~g}, 60.3 \mathrm{mmol}$ ) was added to the flask followed by the addition of THF (approximately $600 \mathrm{~mL}$ ). The nitrosated cyclopentyl derivative of $(1 \mathrm{~S}, 2 R)$ norephedrine $(7.19 \mathrm{~g}, 29.0 \mathrm{mmol})$ was dissolved in THF (100 mL) and added, dropwise, to the flask. The reaction was gently refluxed for three hours, and, then, cooled in an ice bath. Then, $1 \mathrm{M} \mathrm{NaOH}(200 \mathrm{~mL}, 200 \mathrm{mmol})$ was added. Rotary evaporation was used to remove the THF. The product was extracted with ethyl acetate, washed with Rochelle's solution and brine, and dried over $\mathrm{NaSO}_{4}$. The ethyl acetate was 
removed by rotary evaporation resulting in the hydrazine, an oily, yellow product (5.24 g, $22.4 \mathrm{mmol})$.

Cyclization: The hydrazine was dissolved in hexanes (144 mL) in a flame-dried, nitrogenpurged $1 \mathrm{~L}$ round-bottom flask. Diethyl carbonate $(2.98 \mathrm{~mL}, 24.6 \mathrm{mmol})$ was added to the flask and the solution was refluxed under nitrogen for 16 hours. The resulting product was cooled in an ice bath and the hexanes were removed by rotary evaporation. The resulting product was dissolved in ethyl acetate $(123 \mathrm{~mL})$, washed with $1 \mathrm{M} \mathrm{HCl}(3 \mathrm{x}$ $15 \mathrm{~mL}$ ) and brine, and dried over sodium sulfate. Rotary evaporation was used to remove the ethyl acetate and a yellow, oily solid was obtained (5.16 g). Crystallization of the final product was achieved by dissolving the product in ethyl acetate and adding hexanes as an anti-solvent, resulting in a crystalline product $(1.18 \mathrm{~g}, 4.53 \mathrm{mmol})$. The percent yield was $6.85 \%$.

\section{Experimental Procedure for the Intended Synthesis of $(5 R, 6 S)-4-E t h y l-5-m e t h y l-6-$ phenyl-2H-1,3,4-oxadiazinan-2-one, 5}

The synthesis of $(5 R, 6 S)$-4-ethyl-5-methyl-6-phenyl-2H-1,3,4-oxodiazinanan-2ne proved to be problematic. Using acetaldehyde was difficult because it has such a low boiling point that it evaporated before the reaction occurred. Thus acetic anhydride was used, producing an amide derivative which was then reduced by $\mathrm{NaBH}_{4}$ and $\mathrm{I}_{2}$ followed by nitrosation. ${ }^{24}$ This method resulted in a mixture of the amide and amine and other unknown compounds. Two grams of the amide were produced to use for future study. The initial procedure was once again tried with a large excess of acetaldehyde. The acetaldehyde was stored in a freezer at $0{ }^{\circ} \mathrm{C}$ prior to use. Nitrosation followed and flash chromatography was used to purify the nitrosated compound. The ${ }^{1} \mathrm{H}$ NMR spectrum 
indicated the proper compound was made. However, after the product was reduced, cyclized and then purified using flash chromatography, X-ray diffraction analysis determined the structure in Figure 2.10. This structure indicates the cyclization had not been completed. The specifics of the synthesis follow.

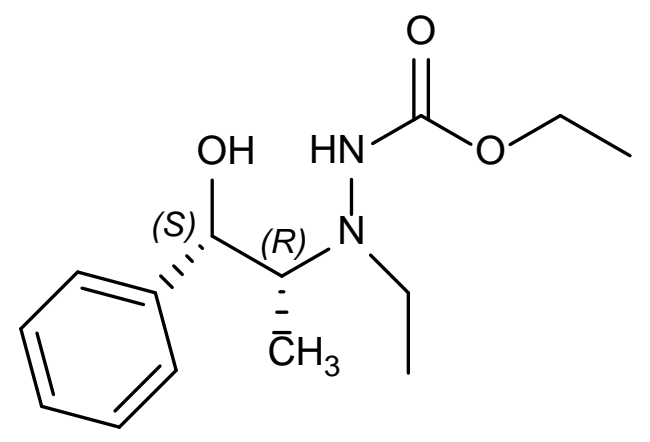

Figure 2.10 Line drawing of 5. This is not an oxadiazinanone, but the product of an oxadiazinanone reaction which did not cyclize.

Alkylation: (1S,2R) norephedrine (10.00 g, $66.13 \mathrm{mmol})$ and acetaldehyde $(7.4 \mathrm{~mL}, 130$ mmol) and ethanol (100 mL) were added to a nitrogen-purged, 1L round-bottom flask. The syringe used to add the acetaldehyde was placed in the freezer before use in an attempt to reduce the evaporation of the aldehyde. The mixture was refluxed for 24 hours and cooled in an ice bath. $\mathrm{NaBH}_{4}(6.24 \mathrm{~g}, 165 \mathrm{mmol})$ was added over a period of approximately 30 minutes. The flask was removed from the ice and ethanol was added (100 mL). After the mixture was stirred for an additional 2.5 hours, $1 \mathrm{M} \mathrm{NaOH}(100 \mathrm{~mL}$, $100 \mathrm{mmol}$ ) was added. Ethanol was removed using rotary evaporation. The resulting product was extracted with ethyl acetate, washed with brine, and dried over sodium 
sulfate resulting, in the ethyl derivative of $(1 S, 2 R)$ norephedrine (11.91 g, $66.50 \mathrm{mmol})$. ${ }^{1} \mathrm{H}$ NMR analysis revealed the product was pure and nitrosation could take place. Nitrosation: The ethyl derivative of $(1 S, 2 R)$ norephedrine was nitrosated by, first, adding 3M HCL ( $25 \mathrm{~mL}, 75 \mathrm{mmol}$ ) to the alkylated product and then adding THF ( $50 \mathrm{~mL}$ ). The mixture was stirred. Sodium nitrite (5.6 g, $79 \mathrm{mmol}$ ) was added and the reaction was allowed to stir overnight. The solution was orange-brown in color. Sodium bicarbonate (saturated solution, $50 \mathrm{~mL}$ ) was added until the solution reached a $\mathrm{pH}$ of 8 . THF was removed by rotary evaporation. The final product was extracted with ethyl acetate, washed with brine, and dried over $\mathrm{Na}_{2} \mathrm{SO}_{4}$. The ethyl acetate was removed by rotary evaporation resulting in the nitrosated ethyl derivative of $(1 S, 2 R)$ norephedrine. ${ }^{1} \mathrm{H}$ NMR spectroscopy indicated the product needed purification. This was accomplished using flash chromatography resulting in the final crystalline product. (3.97 g, $19.1 \mathrm{mmol}$ ). Reduction: Hexanes (200 mL) were added to a flame-dried, nitrogen-purged, 2L roundbottom flask. Lithium aluminum hydride $(0.9737 \mathrm{~g}, 38.15 \mathrm{mmol})$ was added to the flask followed by the addition of THF (600 mL). The nitrosated ethyl derivative of $(1 S, 2 R)$ norephedrine (3.97 g, $19.1 \mathrm{mmol})$ was dissolved in THF (100 mL) and added, drop-wise, to the flask. The reaction was gently refluxed for three hours, cooled in an ice bath, and $1 \mathrm{M} \mathrm{NaOH}$ (200 mL, $200 \mathrm{mmol}$ ) was added. Rotary evaporation was used to remove the THF, and the product was extracted with ethyl acetate andwashed with Rochelle's solution. Then it was washed with brine, and dried over $\mathrm{NaSO}_{4}$. The ethyl acetate was removed by rotary evaporation resulting in the hydrazine, an oily, yellow product (2.49 g, $12.8 \mathrm{mmol})$. 
Cyclization: The hydrazine was dissolved in hexanes $(144 \mathrm{~mL})$ in a flame-dried and nitrogen-purged 1L round-bottom flask. Diethyl carbonate $(1.7 \mathrm{~mL}, 21 \mathrm{mmol})$ was added to the flask and the solution was brought to reflux. Lithium hydride $(0.33 \mathrm{~g}, 42 \mathrm{mmol})$ was added slowly and the solution was refluxed under nitrogen overnight. The resulting product was cooled and hexanes were removed by rotary evaporation. The resulting product was dissolved in ethyl acetate $(123 \mathrm{~mL})$, washed with $1 \mathrm{M} \mathrm{HCl}$, washed with brine, and dried over sodium sulfate. Rotary evaporation was used to remove the ethyl acetate and a yellow, oily compound was obtained ( 5.16 g). The resulting product was dissolved in ethyl acetate and hexanes were added to cause the product to crystallize (2.19 g, $9.95 \mathrm{mmol})$. The percent yield was 15.1\%.

\section{Experimental Procedure for the Intended Synthesis of $(5 R, 6 S)$-4-Ethyl-5-methyl-6- phenyl-2H-1,3,4-oxadianinan-2-one, 6}

In an attempt to synthesize (5R,6S)-4-ethyl-5-methyl-6-phenyl-2H-1,3,4-

oxadiazinanan-2-one using standard literature procedures ${ }^{27}$ the compound depicted in the ChemDraw $^{30}$ figure in Figure 2.11 was synthesized and was identified using X-ray diffraction indicating the cyclization reaction had not gone to completion.

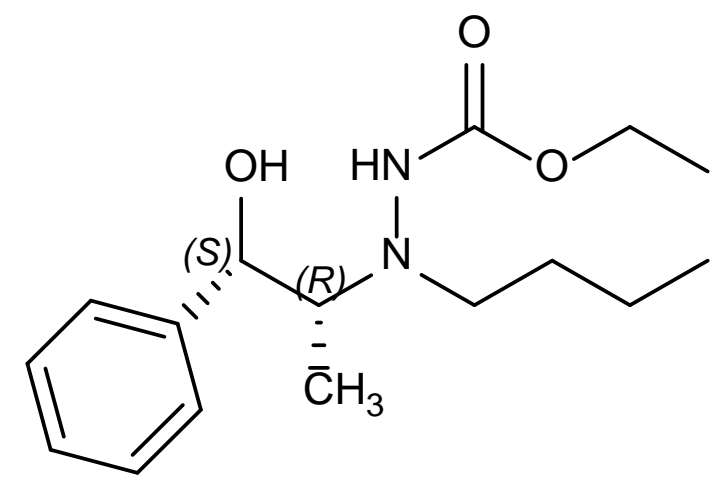

Figure 2.11 Line drawing of $\mathbf{6}$. This is not an oxadiazinanone, but, instead is the product of an oxadiazinanone reaction which did not cyclize. 
Alkylation: (1S,2R) norephedrine (10.00 g, $66.13 \mathrm{mmol})$, butanal $(7.2 \mathrm{~mL}, 80 \mathrm{mmol})$ and ethanol $(100 \mathrm{~mL})$ were added to a nitrogen-purged, $1 \mathrm{~L}$, round-bottom flask. The mixture was refluxed for 24 hours and cooled in an ice bath. $\mathrm{NaBH}_{4}(6.26 \mathrm{~g}, 165 \mathrm{mmol})$ was added over a period of 30 minutes. The flask was removed from the ice and ethanol was added (100 mL). The mixture was stirred for an additional 2.5 hours, and 1M $\mathrm{NaOH}(100$ $\mathrm{mL}, 100 \mathrm{mmol}$ ) was added. Ethanol was removed from the solution using rotary evaporation, and the product was extracted with ethyl acetate, washed with brine, and dried over sodium sulfate resulting in the $n$-butyl derivative of $(1 S, 2 R)$ norephedrine. The product was purified with flash chromatography.

Nitrosation: The $n$-butyl derivative of $(1 S, 2 R)$ norephedrine was nitrosated by dissolving the alkylated norephedrine in $3 \mathrm{M} \mathrm{HCl}(25 \mathrm{~mL}, 75 \mathrm{mmol}$. THF $(60 \mathrm{~mL})$ was added and the mixture was stirred while $\mathrm{NaNO}_{2}(5.5 \mathrm{~g}, 79 \mathrm{mmol})$ was added. The reaction proceeded under reflux overnight. Sodium bicarbonate (saturated solution) was added to the flask until a pH of 8 was reached. Rotary evaporation was used to remove the THF. The final product was extracted with ethyl acetate, washed with brine, and dried over $\mathrm{Na}_{2} \mathrm{SO}_{4}$. The ethyl acetate was removed by rotary evaporation resulting in the nitrosated $n$-butyl derivative of $(1 \mathrm{~S}, 2 \mathrm{R})$ norephedrine. Flash chromatography was used to purify the product (5.13 g, $21.7 \mathrm{mmol})$.

Reduction: Hexanes $(200 \mathrm{~mL})$ were added to a flame-dried, nitrogen-purged, 2L roundbottom flask. Lithium aluminum hydride ( $2.47 \mathrm{~g}, 65.1 \mathrm{mmol}$ ) was added to the flask followed by the addition of THF (135 mL). The nitrosated $n$-butyl derivative of $(1 S, 2 R)$ norephedrine (5.13 g, $21.7 \mathrm{mmol}$ ) was dissolved in THF (50 mL) and added, dropwise, to the flask. The reaction was refluxed for an additional two hours. The reaction was cooled 
in an ice bath and 3M NaOH (40 mL, $100 \mathrm{mmol})$ was added. THF was removed using rotary evaporation. The hydrazine was extracted with ethyl acetate, washed with Rochelle's solution and brine, and dried over $\mathrm{NaSO}_{4}$. The ethyl acetate was removed by rotary evaporation resulting in an oily, yellow product (4.83 g, $21.7 \mathrm{mmol})$. Cyclization: The hydrazine was dissolved in hexanes $(200 \mathrm{~mL})$ in a nitrogen-purged, flame-dried, 1L round-bottom flask. Diethyl carbonate $(2.82 \mathrm{~mL}, 23.8 \mathrm{mmol})$ was added to the flask and the solution was brought to reflux under nitrogen. Lithium hydride (0.35 g, $44 \mathrm{mmol}$ ) was added, and the solution was refluxed overnight. The resulting product was cooled in an ice bath, and hexanes were removed with rotary evaporation. The resulting product was dissolved in ethyl acetate $(123 \mathrm{~mL})$, washed with $1 \mathrm{M} \mathrm{HCl}$, then with brine, and dried over sodium sulfate. Rotary evaporation was used to remove the ethyl acetate, and a yellow, oily solid was obtained (4.41 g). The oil was purified using flash chromatography resulting in a white crystalline product (5.17 mmol, 7.82\% yield).

\section{Crystallization Procedure}

Single crystals were formed using vapor diffusion. If a pure compound was being crystallized, a sample of approximately $0.008 \mathrm{~g}$ was placed in an uncapped inner vial in $0.5 \mathrm{~mL}$ of solvent. If a racemate or quasiracemate was being crystallized, a mixture of the compounds was used with a total weight of approximately $0.008 \mathrm{~g}$. Since the molecular weights of the oxadiazinanones are similar, this provided nearly equimolar solutions with concentrations of approximately $3 \times 10^{-5} \mathrm{M}$ for each component. If just one compound was being crystallized, this provided a $6 \times 10^{-5} \mathrm{M}$ concentration. Into an outer vial was placed $2.5 \mathrm{~mL}$ of the anti-solvent, hexanes (boiling point, $69^{\circ} \mathrm{C}$. The crystals are not soluble in hexanes. The outer vial was then closed, allowing the solvent and anti-solvent 
to equilibrate by vapor diffusion. Ethyl acetate (boiling point, $77^{\circ} \mathrm{C}$ ) was used as the solvent. In this system the hexanes diffuse into the inner vial. At the same time there is a slow diffusion of the solvent out of the inner vial into the hexanes. The oxadiazinanones are not as soluble in hexanes as in ethyl acetate. Because the diffusion taking place in the system increases the concentration of hexanes in the inner vial, crystals slowly form. ${ }^{31}$ If crystallization of the oxadiazinanones did not happen after 2 to 3 days, the hexanes were removed and replaced with fresh hexanes. Usually, crystals formed within 2 to 3 days at room temperature.

\section{X-Ray Diffraction}

X-ray structures were obtained by selecting a suitable crystal using a dissecting microscope. The dimensions of most crystals were approximately $0.2 \mathrm{~mm}$ to $0.4 \mathrm{~mm}$ on each side. The crystals were then mounted using mineral oil as an adherent on MiTeGen Micromounts with loops or MicroMesh aperatures. The mount was attached to the goniometer head and mounted on a Bruker Apex II diffractometer. Mo- $\mathrm{K}_{\alpha}$ radiation ( $\lambda=0.71073 \AA$ ) was used for the collection of the data. The sample was generally kept at 100K during data collection. For some samples, additional information was gained by varying the temperature during collection as indicated in the experimental details about each compound. All data were collected using the software program, SAINT, and refined using SMART $+{ }^{32}$ Solution and data analysis were completed using the WinGX software package. ${ }^{33}$ The structures were solved using direct methods employing the programs, SIR2004 ${ }^{34}$ or SuperFlip. ${ }^{35}$ The refinement was accomplished using SHELXL-2012. ${ }^{36}$ The non-hydrogen atoms were refined anisotropically and, in most cases, the hydrogen atoms were assigned using a riding-model approximation. Hydrogen atoms attached to nitrogen 
atoms were freely refined unless indicated otherwise in the experimental details for each compound. 
CHAPTER III

CRYSTALLOGRAPHIC RESULTS

\section{Crystallographic Details of $(5 R, 6 S)-4-C y c l o p e n t y l-5-m e t h y l-6-p h e n y l-2 H-1,3,4-$ oxadiazinan-2-one, 1}

Figure 3.1 shows a ChemDraw ${ }^{30}$ depiction of $(5 R, 6 S)$-4-cyclopentyl-5-methyl-6phenyl-2H-1,3,4-oxadiazinan-2-one (1).

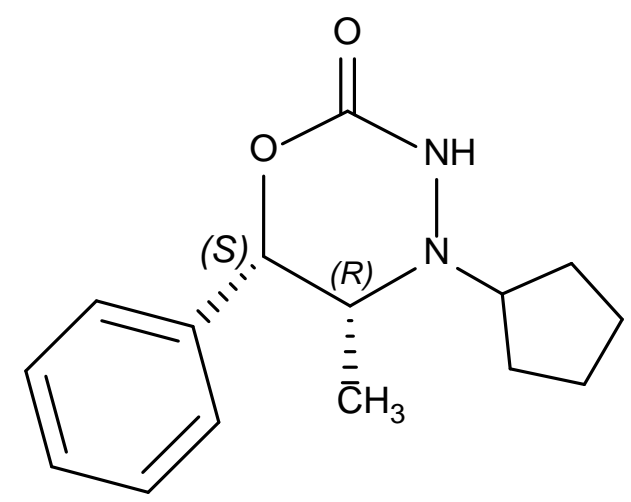

Figure 3.1 Line diagram of 1.

The dimensions of the crystal used for X-ray diffraction were $0.360 \mathrm{~mm}$ x $0.270 \mathrm{~mm} \mathrm{x}$ $0.210 \mathrm{~mm}$. The sample was g kept at 100K during the diffraction experiment. Shelxl$2012^{36}$ was used for refinement. The unit cell parameters were obtained from a leastsquares refinement of 4581 unique reflections out of the 17571 reflections collected. The crystal is orthorhombic and crystallizes in the $P 2{ }_{1} 2_{1} 2_{1}$ space group. The cell lengths are: $a=8.4171(3) \AA, b=9.0618(4) \AA$, and $c=36.7815(13) \AA$. The volume of the cell is 
$2805.47 \AA^{3}$ and $\rho=1.233 \mathrm{~g} / \mathrm{cm}^{3} . Z^{\prime}$ is 2 and $Z$ is 8 . Diffraction data were measured between $\theta_{\min }=1.107^{\circ}$ and $\theta_{\max }=25.352^{\circ}$. The limiting indices were $-10 \leq h \leq 10,-10 \leq k$

$\leq 10$, and $-44 \leq l \leq 44$. All hydrogen atoms attached to nitrogen were freely refined and all other hydrogens were refined using the riding-model approximation. Convergence was reached on the full-matrix least-squares refinement of $F^{2}$. The $R^{1}$-factor is 0.0432 and $w R^{2}$ is 0.1029. Figure 3.2 shows an ORTEP $\mathrm{P}^{33}$ depiction of $\mathbf{1}$. The ellipsoids are at $50 \%$ and the hydrogen atoms have been omitted for clarity. The hydrogen bonds are shown as dotted lines.

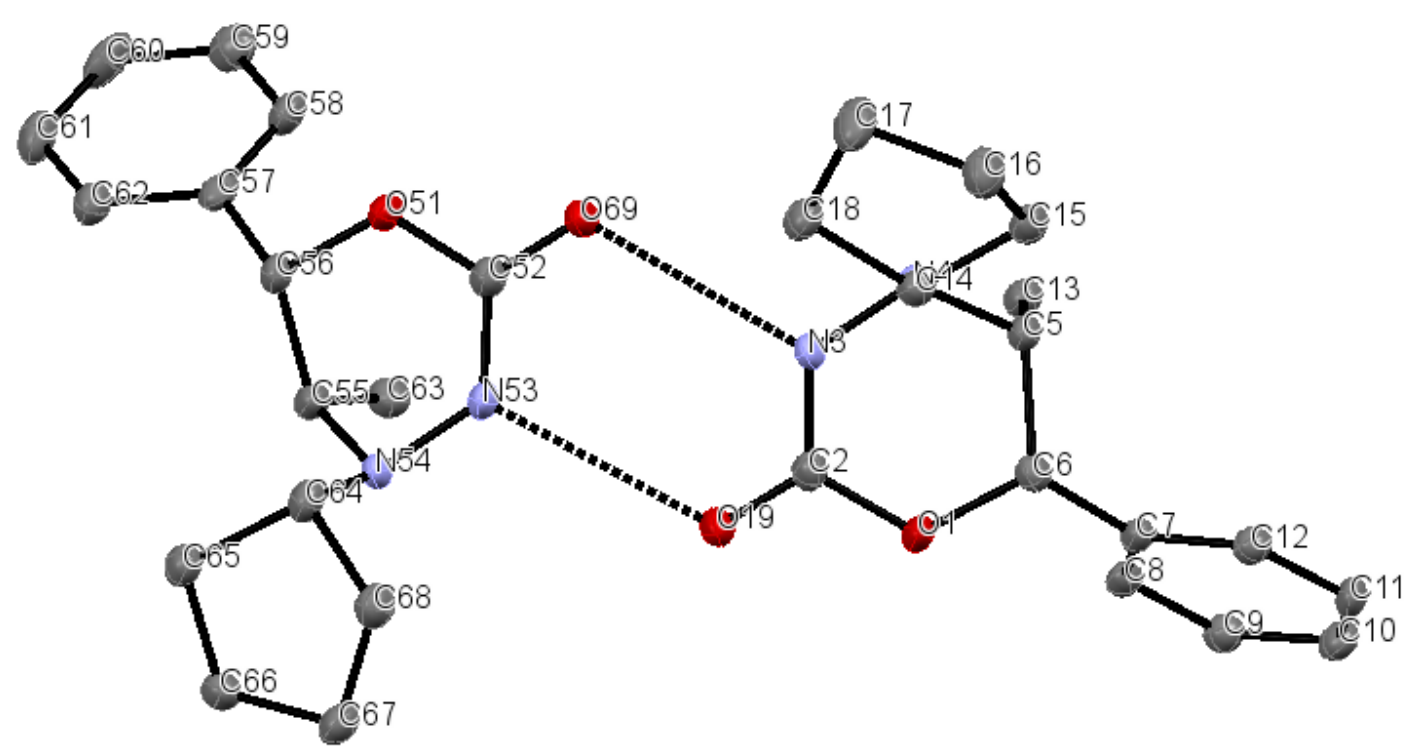

Figure 3.2 Ellipsoid depiction of $\mathbf{1}$.

The bond lengths, bond angles and torsion angles of the central oxadiazinanone ring as well as any carbon atoms attached to the ring are shown in Tables 3.1-3. Table 3.4 
shows the hydrogen bond lengths. Molecule 1 refers to the molecule with the lower range of atom numbers. This is the molecule on the right side in Figure 3.2.

Table 3.1 Bond lengths of the central oxadiazinanone rings in the asymmetric unit of $\mathbf{1}$.

\begin{tabular}{llll}
\hline Atoms (Molecule 1) & Distance $(\AA)$ & Atoms (Molecule 2) & Distance $(\AA)$ \\
\hline O1-C2 & $1.356(4)$ & O51-C52 & $1.356(4)$ \\
O1-C6 & $1.463(4)$ & O51-C56 & $1.455(4)$ \\
C2-O19 & $1.223(4)$ & C52-O69 & $1.224(4)$ \\
C2-N3 & $1.336(4)$ & C52-N53 & $1.338(4)$ \\
N3-N4 & $1.424(4)$ & N53-N54 & $1.428(4)$ \\
N4-C14 & $1.479(4)$ & N54-C64 & $1.490(4)$ \\
N4-C5 & $1.470(4)$ & N54-C55 & $1.466(4)$ \\
C5-C6 & $1.542(5)$ & C55-C56 & $1.534(5)$ \\
C5-C13 & $1.516(5)$ & C55-C63 & $1.517(5)$ \\
C6-C7 & $1.499(5)$ & C56-C57 & $1.507(5)$ \\
\hline
\end{tabular}

Table 3.2 Bond angles of the central oxadiazinanone rings in the asymmetric unit of $\mathbf{1}$.

\begin{tabular}{lcll}
\hline Atoms (Molecule 1) & Angle $\left(^{\circ}\right)$ & Atoms (Molecule 2) & Angle $\left(^{\circ}\right)$ \\
\hline C6-O1-C2 & $121.1(3)$ & C56-O51-C52 & $121.6(3)$ \\
O1-C2-N3 & $118.7(3)$ & O51-C52-N53 & $118.6(3)$ \\
O1-C2-O19 & $117.5(3)$ & O51-C52-O69 & $117.9(3)$ \\
O19-C2-N3 & $123.8(3)$ & O69-C52-N53 & $123.5(3)$ \\
C2-N3-N4 & $125.9(3)$ & C52-N53-N54 & $124.7(3)$ \\
N3-N4-C5 & $108.5(2)$ & N53-N54-C55 & $107.0(2)$ \\
C14-N4-C5 & $114.9(3)$ & C64-N54-C55 & $117.0(3)$ \\
N4-C5-C13 & $109.4(3)$ & N54-C55-C63 & $110.0(3)$ \\
C13-C5-C6 & $114.3(3)$ & C63-C55-C56 & $114.1(3)$ \\
\hline
\end{tabular}


Table 3.3 Torsion angles of the central oxadiazinanone rings in the asymmetric unit of $\mathbf{1}$.

\begin{tabular}{llll}
\hline Atoms (Molecule 1) & Torsion $\left(^{\circ}\right)$ & Atoms (Molecule 2) & Torsion ( $\left.{ }^{\circ}\right)$ \\
\hline C6-O1-C2-N3 & $1.5(4)$ & C56-O51-C52-N53 & $-7.4(5)$ \\
C6-O1-C2-O19 & $179.1(3)$ & C56-O51-C52-O69 & $172.0(3)$ \\
C2-O1-C6-C5 & $-26.7(4)$ & C52-O51-C56-C55 & $-20.4(4)$ \\
C2-O1-C6-C7 & $-151.2(3)$ & C52-O51-C56-C57 & $-143.8(3)$ \\
O1-C2-N3-N4 & $-4.1(5)$ & O51-C52-N53-N54 & $1.1(5)$ \\
O19-C2-N3-N4 & $178.4(3)$ & O69-C52-N53-N54 & $-178.3(3)$ \\
C2-N3-N4-C5 & $32.5(4)$ & C52-N53-N54-C55 & $33.0(4)$ \\
C2-N3-N4-C14 & $-92.5(4)$ & C52-N53-N54-C64 & $-94.1(4)$ \\
N3-N4-C5-C6 & $-55.3(3)$ & N53-N54-C55-C56 & $-58.8(3)$ \\
N3-N4-C5-C13 & $70.7(3)$ & N53-N54-C55-C63 & $67.4(3)$ \\
C14-N4-C5-C6 & $65.8(3)$ & C64-N54-C55-C56 & $63.1(3)$ \\
C14-N4-C5-C13 & $-168.2(3)$ & C64-N54-C55-C63 & $-170.8(3)$ \\
N4-C5-C6-O1 & $54.1(3)$ & N54-C55-C56-O51 & $54.1(3)$ \\
N4-C5-C6-C7 & $175.0(3)$ & N54-C55-C56-C57 & $175.2(3)$ \\
\hline
\end{tabular}

Table 3.4 Donor-Acceptor Hydrogen bond lengths in $\mathbf{1 .}$

\begin{tabular}{lll}
\hline Donor-Acceptor & Distance, D-A (̊̊) & Bonding Motif \\
\hline N53-O19 & 2.879 & Different Enantiomorph \\
O69-N3 & 2.888 & \\
\hline
\end{tabular}

\section{Crystallographic Details of Racemic 4-Cyclopentyl-5-methyl-6-phenyl-2H-1,3,4- oxadiazinan-2-one, 2}

Compound 2 was prepared by combining equal amounts of each enantiomer of 4cyclopentyl-5-methyl-6-phenyl-2H-1,3,4-oxadiazinan-2-one. The two enantiomers are shown in Figure 3.3. The enantiomer on the left is the ChemDraw ${ }^{30}$ depiction of $(5 R, 6 S)$ configuration and on the right is the ChemDraw ${ }^{30}$ depiction of $(5 S, 6 R)$ enantiomer. 

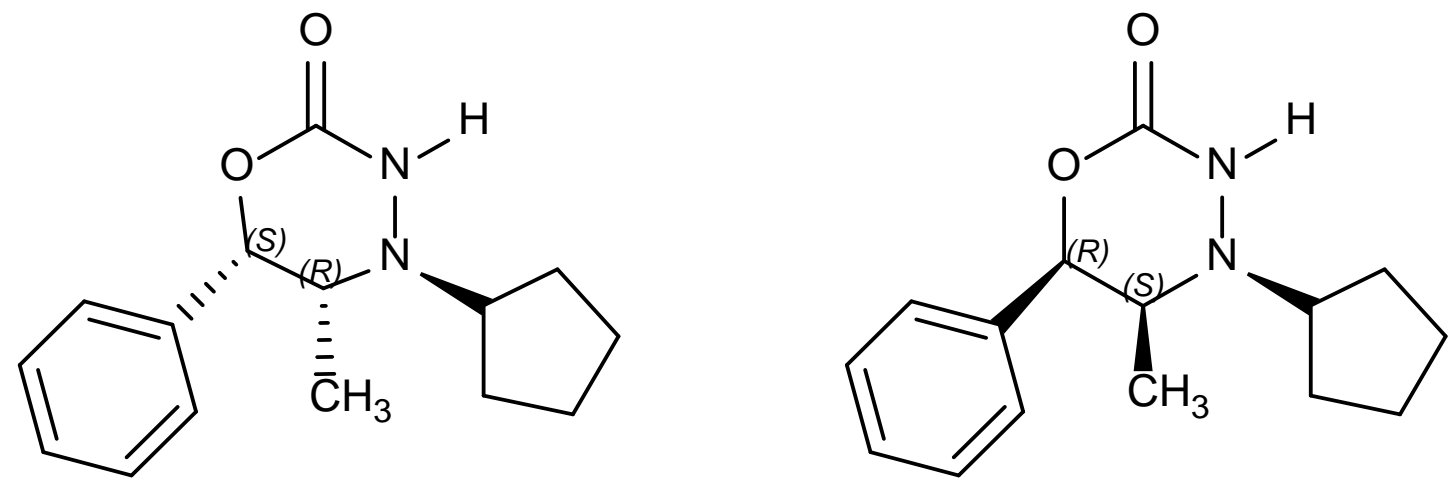

Figure 3.3 Line drawings of the two enantiomers of 2.

The temperature was $292 \mathrm{~K}$. Shelxl-2012 ${ }^{36}$ was used for refinement. The unit cell parameters were obtained from a least-squares refinement of 2460 unique reflections out of the 19297 reflections collected. The crystal is monoclinic and crystallizes in the $P 2_{1} / C$ space group. The cell lengths are: $a=9.1808(3) \AA, b=15.7560(5) \AA$, and $c=9.7293(3)$ A. The angles of the cell are: $\alpha=90^{\circ}, \beta=106.132(2)^{\circ}$, and $\gamma=90^{\circ}$. The volume of the cell is $1351.95 \AA^{3}$ and $\rho=1.279 \mathrm{~g} / \mathrm{cm}^{3}$. $Z^{\prime}$ is 1 and $Z$ is 4 . Diffraction data were measured between $\theta_{\min }=2.309^{\circ}$ and $\theta_{\max }=25.300^{\circ}$. The limiting indices were $-11 \leq h \leq$ $11,-18 \leq k \leq 18$, and $-11 \leq l \leq 11$. All hydrogen atoms attached to nitrogen were freely refined, and all other hydrogens were refined using the riding-model approximation. Convergence was reached on the full-matrix least squares refinement of $F^{2}$. The $R^{1}$ factor is 0.0381 and $w R^{2}$ is 0.0926 .

Figure 3.4 shows an ORTEP ${ }^{33}$ depiction of 2 . The ellipsoids are at $50 \%$ and the hydrogen atoms have been omitted for clarity. The hydrogen bonds are shown as dotted lines. 


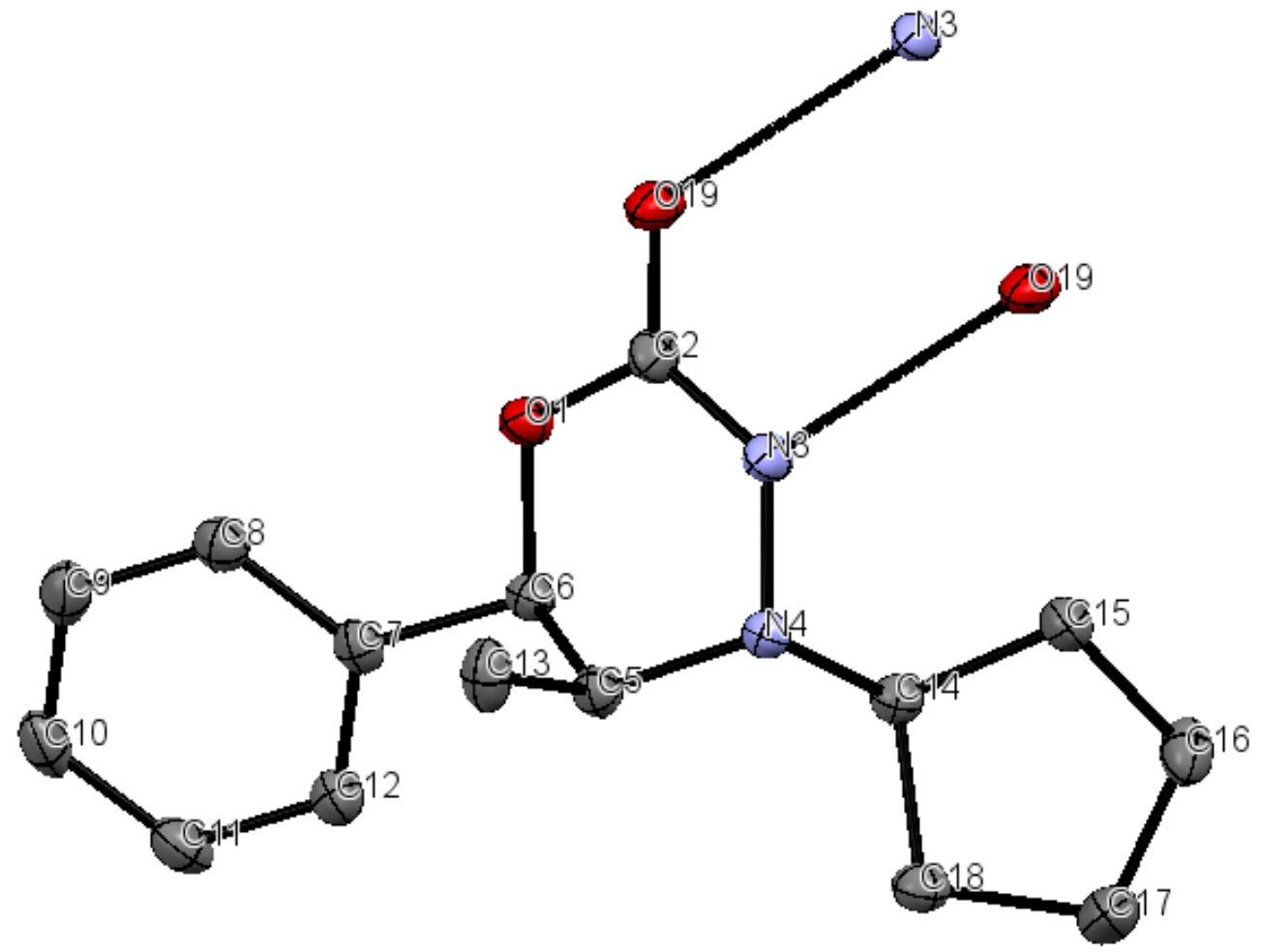

Figure 3.4 Ellipsoid depiction of asymmetric unit of 2.

The bond lengths, bond angles and torsion angles of the central oxadiazinanone ring as well as any carbon atoms attached to the ring are shown in Tables 3.5- 3.7. Each oxadiazinanone structure has two hydrogen bonds in the asymmetric unit. Hydrogen bond distances and angles are shown in Table 3.8. 
Table 3.5 Bond lengths in the central oxadiazinanone ring of 2 .

\begin{tabular}{ll}
\hline Atoms & Bond Length $(\AA \AA)$ \\
\hline O1-C2 & $1.354(2)$ \\
O1-C6 & $1.446(2)$ \\
C2-N3 & $1.333(2)$ \\
C2-O20 & $1.227(2)$ \\
N3-N4 & $1.429(2)$ \\
N4-C5 & $1.473(2)$ \\
C5-C6 & $1.533(2)$ \\
C7-C8 & $1.391(3)$ \\
\hline
\end{tabular}

Table 3.6 Bond angles of the central oxadiazinanone ring of $\mathbf{2}$.

\begin{tabular}{ll}
\hline Atoms & Angle $\left(^{\circ}\right)^{\circ}$ \\
\hline C6-O1-C2 & $118.5(1)$ \\
O1-C2-N3 & $118.8(1)$ \\
O1-C2-O19 & $117.7(1)$ \\
O19-C2-N3 & $123.4(1)$ \\
C2-N3-N4 & $127.5(1)$ \\
N3-N4-C5 & $108.7(1)$ \\
C14-N4-C5 & $114.9(1)$ \\
N4-C5-C13 & $109.9(1)$ \\
C13-C5-C6 & $113.0(1)$ \\
C5-C6-O1 & $108.9(1)$ \\
\hline
\end{tabular}


Table 3.7 Torsion angles of the central oxadiazinanone ring in the asymmetric unit of 2 .

\begin{tabular}{ll}
\hline Atoms & Torsion $\left(^{\circ}\right)$ \\
\hline C6-O1-C2-N3 & $-7.7(2)$ \\
C6-O1-C2-O20 & $174.5(1)$ \\
C2-O1-C6-C5 & $40.0(2)$ \\
C2-O1-C6-C7 & $165.4(1)$ \\
O1-C2-N3-N4 & $-6.4(2)$ \\
O19-C2-N3-N4 & $171.3(1)$ \\
C2-N3-N4-C5 & $-15.3(2)$ \\
C2-N3-N4-C14 & $111.3(2)$ \\
N3-N4-C5-C6 & $47.0(1)$ \\
N3-N4-C5-C13 & $-77.9(1)$ \\
C14-N4-C5-C6 & $-76.6(1)$ \\
C14-N4-C5-C13 & $158.5(1)$ \\
N4-C5-C6-O1 & $-60.4(1)$ \\
N4-C5-C6-C7 & $178.8(1)$ \\
C13-C5-C6-O1 & $62.7(1)$ \\
C13-C5-C6-C7 & $-58.1(2)$ \\
\hline
\end{tabular}

Table 3.8 Donor-Acceptor Hydrogen bond lengths in 2.

\begin{tabular}{ccc}
\hline $\begin{array}{c}\text { Donor- } \\
\text { Acceptor }\end{array}$ & Distance, D-A $(\AA)$ & Bonding Motif \\
\hline N3-O19 & 2.824 & Same enantiomorph \\
\hline
\end{tabular}

\section{Crystallographic Details of Quasiracemate of (5R,6S)-4-Cyclopentyl-5-methyl-6- phenyl-2H-1,3,4-oxadiazinan-2-one and $(5 S, 6 R)-4$-Isopropyl- 5-methyl-6-phenyl-2H-1,3,4-oxadiazinan-2-one, 3}

ChemDraw $^{30}$ depictions of the quasienantiomers used to produce $\mathbf{3}$ are shown in Figure 3.5. X-ray diffraction was used to confirm the presence of quasiracemates. 

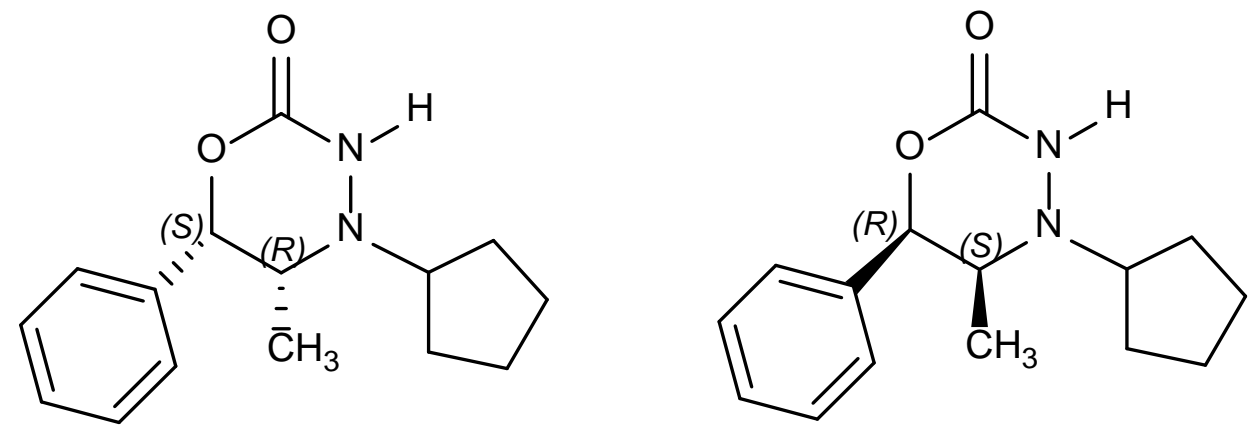

Figure 3.5 Line drawings of (left) $(5 R, 6 S)$-4-cyclopentyl-5-methyl-6-phenyl-2H-1,3,4oxadiazinan-2-one and (right) (5S,6R)-4-cyclopentyl-5-methyl -6- phenyl-2H-3,4oxadiazinan-2-one.

The temperature was $292 \mathrm{~K}$. Shelxl-2012 ${ }^{36}$ was used for refinement. The unit cell parameters were obtained from a least-squares refinement of 8339 unique reflections out of the 52929 reflections collected. The crystal is monoclinic and crystallizes in the $P 2_{1}$ space group. The cell lengths are: $a=10.4323(3) \AA, b=8.7068(2) \AA$, and $c=14.6212(4) \AA$. The volume of the cell is $1324.64(6) \AA^{3}$ and $\rho=1.240 \mathrm{~g} / \mathrm{cm}^{3}$. The angles of the cell are: $\alpha=90^{\circ}, \beta=94.1200(10)^{\circ}$, and $\gamma=90^{\circ} . Z^{\prime}$ is 2 and $Z$ is 2 . Diffraction data were measured between $\theta_{\min }=1.396^{\circ}$ and $\theta_{\max }=32.151^{\circ}$. The limiting indices were $-15 \leq h \leq 15,-12 \leq k \leq 12$, and $-21 \leq l \leq 21$. All hydrogen atoms attached to nitrogen were freely refined and all other hydrogens were refined using the riding-model approximation. Convergence was reached on the full-matrix least-squares refinement on $F^{2}$. The $R^{1}$-factor is 0.0427 and $w R^{2}$ is 0.1098 . An ORTEP ${ }^{33}$ depiction in Figure 3.6. In each figure the hydrogen atoms have been omitted for clarity and hydrogen bonds are shown as dotted lines. In Figure 3.9, the ellipsoids are modeled at 50\% probability. 


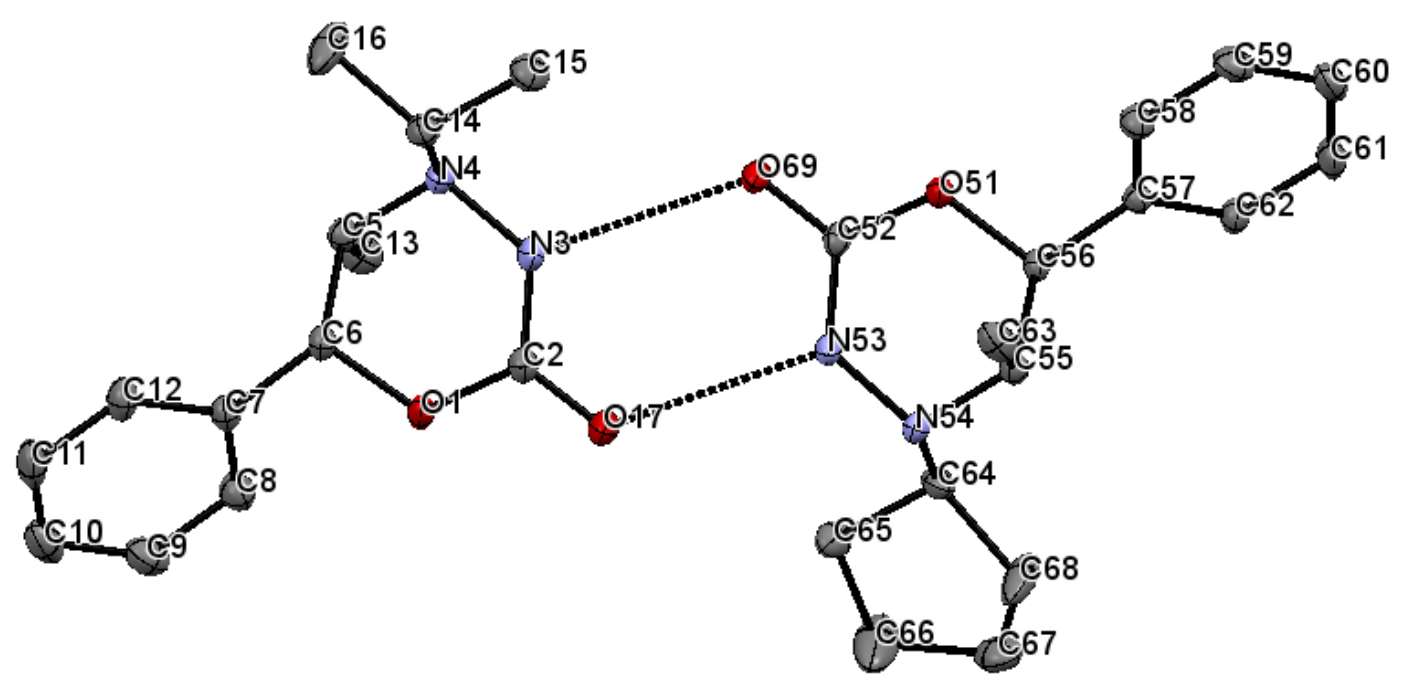

Figure 3.6 Ellipsoid depiction of $\mathbf{3}$.

The bond lengths and bond angles of the central oxadiazinanone ring of $\mathbf{3}$ are shown in Tables 3.9 and 3.10. In Table 3.11 are found the torsions of the central oxadiazinanone ring, as well as the carbons attached to the central ring. Table 3.12 shows the hydrogen bond distances and angles.

Table 3.9 Bond lengths of the central oxadiazinanone atoms of $\mathbf{3}$.

\begin{tabular}{llll}
\hline Atoms-isopropyl & Bond Length $(\AA)$ & Atoms-cyclopentyl & Bond Length $(\AA)$ \\
\hline O1-C2 & $1.353(2)$ & O51-C52 & $1.354(2)$ \\
O1-C6 & $1.457(2)$ & O51-C56 & $1.458(2)$ \\
C2-N3 & $1.342(2)$ & C52-N53 & $1.338(2)$ \\
C2-O17 & $1.228(2)$ & C52-O69 & $1.233(2)$ \\
N3-N4 & $1.426(2)$ & N53-N54 & $1.424(2)$ \\
N4-C5 & $1.474(2)$ & N54-C55 & $1.476(2)$ \\
C5-C6 & $1.535(2)$ & C55-C56 & $1.534(2)$ \\
\hline
\end{tabular}


Table 3.10 Bond angles of the central oxadiazinanone rings in the asymmetric unit of 3 .

\begin{tabular}{llll}
\hline Atoms-isopropyl & Angle $\left.\mathbf{(}^{\circ}\right)$ & Atoms-cyclopentyl & Angle $\left(^{\circ}\right)$ \\
\hline C6-O1-C2 & $120.2(1)$ & C56-O51-C52 & $118.6(1)$ \\
O1-C2-N3 & $119.0(1)$ & O51-C52-N53 & $119.1(1)$ \\
O1-C2-O17 & $117.6(1)$ & O51-C52-O69 & $117.8(1)$ \\
O17-C2-N3 & $123.4(2)$ & O69-C52-N53 & $123.1(2)$ \\
C2-N3-N4 & $125.8(1)$ & C52-N53-N54 & $126.8(1)$ \\
N3-N4-C5 & $107.8(1)$ & N53-N54-C55 & $108.4(1)$ \\
C14-N4-C5 & $115.5(1)$ & C64-N54-C55 & $113.7(1)$ \\
N4-C5-C13 & $110.2(1)$ & N54-C55-C63 & $110.7(1)$ \\
C13-C5-C6 & $113.5(1)$ & C63-C55-C56 & $113.5(1)$ \\
C5-C6-O1 & $109.1(1)$ & C55-C56-O51 & $108.6(1)$ \\
\hline
\end{tabular}

Table 3.11 Torsion angles of the central rings in the asymmetric unit of $\mathbf{3}$.

\begin{tabular}{llll}
\hline Atoms-isopropyl & Torsion $\left(^{\circ}\right)$ & Atoms-cyclopentyl & Torsion $\left(^{\circ}\right)$ \\
\hline C6-O1-C2-N3 & $1.6(2)$ & C56-O51-C52-N53 & $7.2(2)$ \\
C6-O1-C2-O17 & $-177.9(1)$ & C56-O51-C52-O69 & $-174.6(1)$ \\
C2-O1-C6-C5 & $29.3(2)$ & C52-O51-C56-C55 & $-38.4(2)$ \\
C2-O1-C6-C7 & $155.9(1)$ & C52-O51-C56-C57 & $-164.9(1)$ \\
O1-C2-N3-N4 & $-4.1(2)$ & O51-C52-N53-N54 & $2.4(2)$ \\
O17-C2-N3-N4 & $175.3(2)$ & O69-C52-N53-N54 & $-175.7(1)$ \\
C2-N3-N4-C5 & $-25.9(2)$ & C52-N53-N54-C55 & $21.7(2)$ \\
C2-N3-N4-C14 & $101.1(2)$ & C52-N53-N54-C64 & $-103.1(2)$ \\
N3-N4-C5-C6 & $55.1(2)$ & N53-N54-C55-C56 & $-51.6(2)$ \\
N3-N4-C5-C13 & $-70.0(2)$ & N53-N54-C55-C63 & $73.7(2)$ \\
C14-N4-C5-C6 & $-68.9(2)$ & C64-N54-C55-C56 & $71.0(2)$ \\
C14-N4-C5-C13 & $166.0(1)$ & C64-N54-C55-C63 & $-163.7(1)$ \\
N4-C5-C6-O1 & $-58.2(2)$ & N54-C55-C56-O51 & $61.2(2))$ \\
N4-C5-C6-C7 & $-179.4(1)$ & N54-C55-C56-C57 & $-176.7(1)$ \\
C13-C5-C6-O1 & $64.9(2)$ & C63-C55-C56-O51 & $-62.4(2)$ \\
C13-C5-C6-C7 & $-56.3(2)$ & C63-C55-C56-C57 & $59.6(2)$ \\
\hline
\end{tabular}


Table 3.12 Donor-Acceptor Hydrogen bond lengths in 3.

\begin{tabular}{lll}
\hline $\begin{array}{c}\text { Donor- } \\
\text { Acceptor }\end{array}$ & Distance, D-A (̊) & \multicolumn{1}{c}{ Bonding Motif } \\
\hline O17-N53 & 2.868 & Different \\
N3-O69 & 2.895 & Enantiomorph \\
\hline
\end{tabular}

\section{Crystallographic Details of Quasiracemate of (5R,6S)-4-Cyclopentyl-5-methyl-6- phenyl-2H-1,3,4-oxadiazinan-2-one and (5S,6R)-4-Cyclohexyl-5-methyl- 6-phenyl-2H-1,3,4-oxadiazinan-2-one, 4}

Shelxl-2012 ${ }^{36}$ was used for refinement. The crystal dimensions are $0.330 \mathrm{~mm} \mathrm{x}$ $0.120 \mathrm{~mm} \times 0.100 \mathrm{~mm}$. The temperature was $100 \mathrm{~K}$. The unit cell parameters were obtained from a least-square refinement of 7852 unique reflections out of the 53541 reflections collected. The crystal is monoclinic and crystallizes in the $P 2_{1}$ space group. The cell lengths are: $a=8.8830(3) \AA, b=16.9145(5) \AA$, and $c=10.0167(3) \AA$. The angles of the cell are $\alpha=90^{\circ}, \beta=106.432(2)^{\circ}$, and $\gamma=90^{\circ}$. The volume of the cell is 1443.55(8) $\AA^{3}$ and $\rho=1.230 \mathrm{~g} / \mathrm{cm}^{3} . Z^{\prime}$ is 2 and $Z$ is 8. Diffraction data were measured between $\theta_{\min }=2.120^{\circ}$ and $\theta_{\max }=29.290^{\circ}$. . The limiting indices were $-12 \leq h \leq 12,-23 \leq$ $k \leq 23$, and $-13 \leq l \leq 13$. All hydrogen atoms attached to nitrogen were freely refined and all other hydrogen atoms were refined using the riding-model approximation. Convergence was reached on the full-matrix least-squares refinement on $F^{2}$. The $R^{1}$ factor is 0.0455 and $w R^{2}$ is 0.1126 .

ChemDraw $^{30}$ depictions of the two compounds used to produce $\mathbf{4}$ are shown in Figure 3.7. 

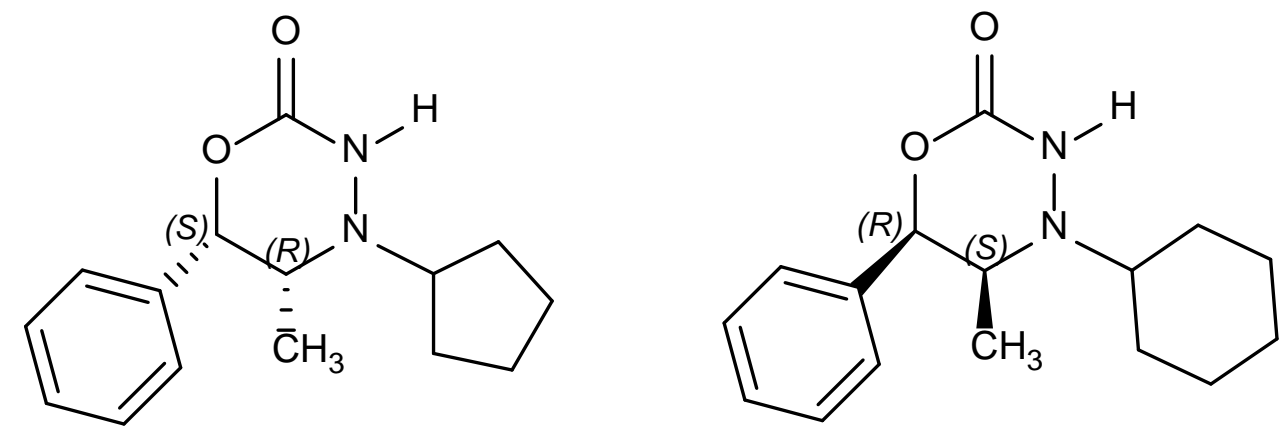

Figure 3.7 (5S,6R)-4-Cyclohexyl-5-methyl-6-phenyl-2H-1,3,4-oxadiazinan-2-one (left) and $(5 R, 6 S)$-5-methyl-4-cyclopentyl-6-phenyl-2H-1,3,4-oxadiazinan-2-one (right).

An ORTEP ${ }^{33}$ depiction of $\mathbf{4}$ can be viewed in Figure 3.8. In each figure the hydrogen atoms have been omitted for clarity and hydrogen bonds are shown as dotted lines. The ellipsoids are modeled at 50\% probability.

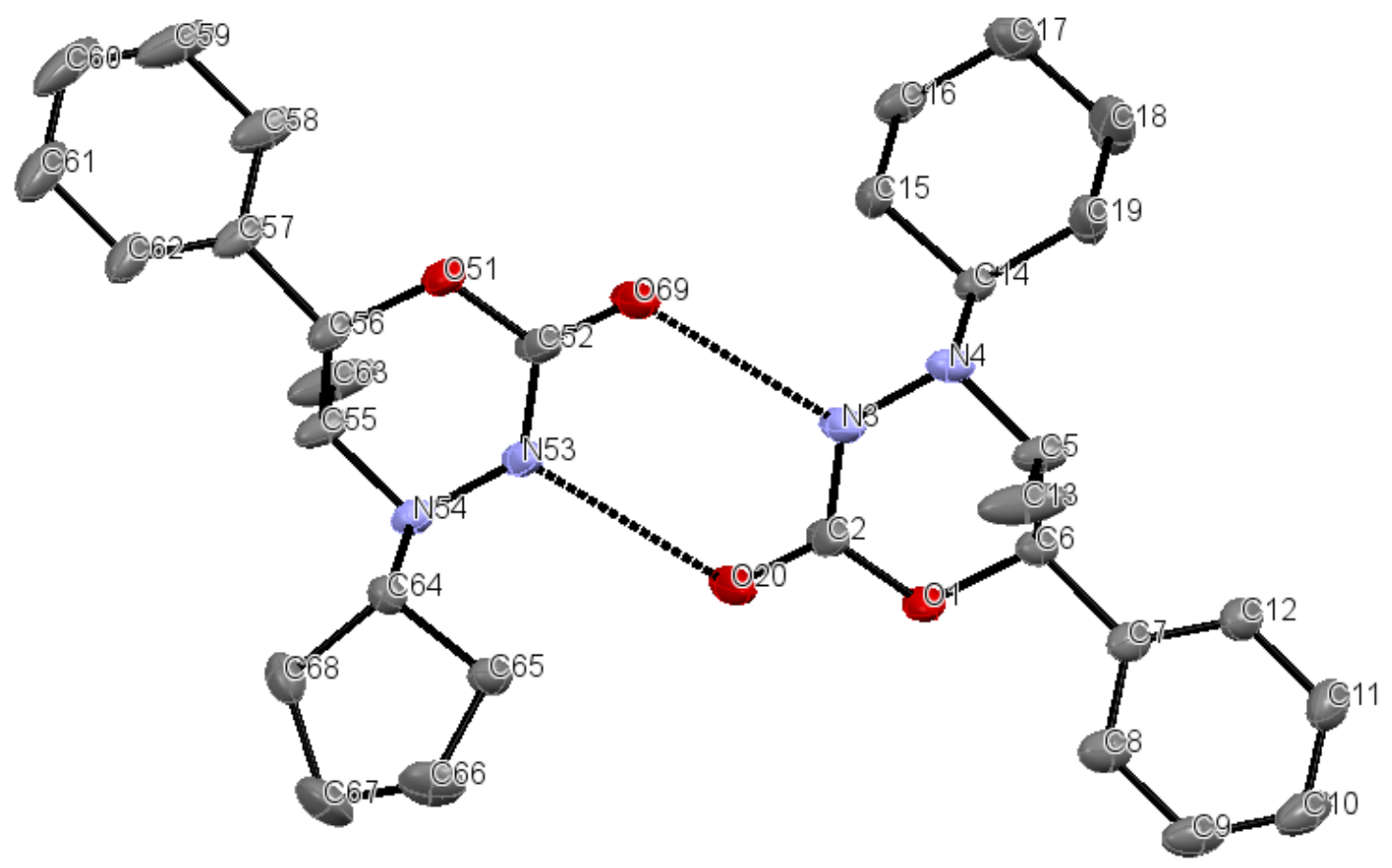

Figure 3.8. Ellipsoid diagram of 4 . 
The bond lengths, bond angles and torsions of the central oxadiazinanone ring are shown in Tables 3.13-3.14. The torsions of the oxadiazinanone ring as well as carbons attached to the central ring are given in Table 3.11. The hydrogen bond distances and angles are displayed in Table 3.15.

Table 3.13 Bond lengths in the central oxadiazinanone ring of 4 .

\begin{tabular}{|c|c|c|c|}
\hline Atoms-Cyclohexyl & Bond Length ( $\AA$ ) & Atoms-Cyclopentyl & Bond Length ( $\AA$ ) \\
\hline $\mathrm{O} 1-\mathrm{C} 2$ & $1.356(3)$ & O51-C52 & $1.353(3)$ \\
\hline O1-C6 & $1.442(3)$ & O51-C56 & $1.446(3)$ \\
\hline C2-N3 & $1.337(3)$ & C52-N53 & $1.343(3)$ \\
\hline $\mathrm{C} 2-\mathrm{O} 20$ & $1.224(4)$ & C52-O69 & $1.225(4)$ \\
\hline N3-N4 & $1.422(3)$ & N53-N54 & $1.429(3)$ \\
\hline N4-C5 & $1.471(4)$ & N54-C55 & $1.476(3)$ \\
\hline C5-C6 & $1.533(3)$ & C55-C56 & $1.534(3)$ \\
\hline C7-C8 & 1.391(3) & C57-C58 & $1.393(4)$ \\
\hline
\end{tabular}

Table 3.14 Bond angles in the central oxadiazinanone rings of 4 .

\begin{tabular}{cccc}
\hline $\begin{array}{c}\text { Atoms- } \\
\text { Cyclohexyl }\end{array}$ & Angle ( $\left.{ }^{\mathbf{0}}\right)$ & Atoms-Cyclopentyl & Angle ( ${ }^{\mathbf{}}$ ) \\
\hline C6-O1-C2 & $118.6(2)$ & C56-O51-C52 & $118.9(2)$ \\
O1-C2-N3 & $119.1(2)$ & O51-C52-N53 & $118.7(2)$ \\
O1-C2-O20 & $117.6(2)$ & O51-C52-O69 & $118.3(2)$ \\
O17-C2-N3 & $123.3(2)$ & O69-C52-N53 & $123.0(2)$ \\
C2-N3-N4 & $126.8(2)$ & C52-N53-N54 & $126.9(2)$ \\
N3-N4-C5 & $108.0(2)$ & N53-N54-C55 & $109.2(2)$ \\
C14-N4-C5 & $116.1(2)$ & C64-N54-C55 & $113.9(2)$ \\
N4-C5-C13 & $110.0(2)$ & N54-C55-C63 & $110.0(2)$ \\
\hline
\end{tabular}


Table 3.15 Torsion angles of the central oxadiazinanone rings of 4 .

\begin{tabular}{llll}
\hline Atoms-Cyclohexyl & \multicolumn{1}{c}{ Torsion $\left(^{\left({ }^{\circ}\right)}\right.$} & Atoms-Cyclopentyl & \multicolumn{1}{c}{ Torsion ( $\left.{ }^{\circ}\right)$} \\
\hline C6-O1-C2-N3 & $-5.1(3)$ & C56-O51-C52-N53 & $7.2(2)$ \\
C6-O1-C2-O20 & $177.0(2)$ & C56-O51-C52-O69 & $-174.6(1)$ \\
C2-O1-C6-C5 & $37.8(3)$ & C52-O51-C56-C55 & $-38.4(2)$ \\
C2-O1-C6-C7 & $163.5(2)$ & C52-O51-C56-C57 & $-164.7(2)$ \\
O1-C2-N3-N4 & $-5.5(4)$ & O51-C52-N53-N54 & $4.1(4)$ \\
O20-C2-N3-N4 & $172.3(2)$ & O69-C52-N53-N54 & $-173.9(2)$ \\
C2-N3-N4-C5 & $-19.6(3)$ & C52-N53-N54-C55 & $18.3(3)$ \\
C2-N3-N4-C14 & $108.1(3)$ & C52-N53-N54-C64 & $-107.3(3)$ \\
N3-N4-C5-C6 & $51.2(2)$ & N53-N54-C55-C56 & $-48.3(2)$ \\
N3-N4-C5-C13 & $-74.0(2)$ & N53-N54-C55-C63 & $76.7(2)$ \\
C14-N4-C5-C6 & $-73.0(2)$ & C64-N54-C55-C56 & $75.0(2)$ \\
C14-N4-C5-C13 & $161.9(2)$ & C64-N54-C55-C63 & $-160.0(2)$ \\
N4-C5-C6-O1 & $-61.8(2)$ & N54-C55-C56-O51 & $59.9(3))$ \\
N4-C5-C6-C7 & $177.0(2)$ & N54-C55-C56-C57 & $-178.7(2)$ \\
C13-C5-C6-O1 & $61.3(3)$ & C63-C55-C56-O51 & $-63.0(3)$ \\
C13-C5-C6-C7 & $-59.9(3)$ & C63-C55-C56-C57 & $58.4(3)$ \\
\hline
\end{tabular}

Table 3.16 Donor-Acceptor Hydrogen bond lengths in 4.

\begin{tabular}{lll}
\hline \multicolumn{1}{c}{$\begin{array}{c}\text { Donor- } \\
\text { Acceptor }\end{array}$} & Distance, D-A (Å) & \multicolumn{1}{c}{ Bonding Motif } \\
\hline N3-O69 & 2.823 & Different \\
O20-N53 & 2.859 & Enantiomorph \\
\hline
\end{tabular}

\section{Crystallographic Details of 5}

In Figure 3.9 is a ChemDraw ${ }^{30}$ depiction of the product which resulted from the attempted synthesis of (5R,6S)-4-ethyl-5-methyl-6-phenyl-3H-1,3,4-oxadiazinan-2-one. 


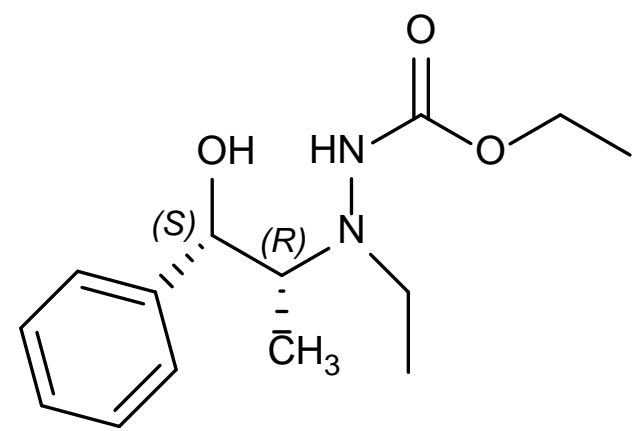

Figure 3.9 Line drawing of 5.

The dimensions of the crystal used for X-ray diffraction were $0.248 \mathrm{~mm}$ x 0.201 $\mathrm{mm} \times 0.061 \mathrm{~mm}$. The sample was generally kept at $100 \mathrm{~K}$. Superflip ${ }^{37}$ was used for solving the structure and Shelxl-2012 ${ }^{36}$ was used for refinement. The unit cell parameters were obtained from a least-squares refinement of 5033 unique reflections out of the 15264 reflections collected. The crystal is orthorhombic and crystallizes in the $P 2{ }_{1} 2_{1} 2_{1}$ space group. The cell lengths are: $a=7.0046(8) \AA, b=10.0479(11) \AA$, and $c=20.587(2)$ $\AA$. The volume of the cell is $1448.94 \AA^{3}$ and $\rho=1.221 \mathrm{~g} / \mathrm{cm}^{3}$. Z' is 1 and $Z$ is 4 . Diffraction data were measured between $\theta_{\min }=1.978^{\circ}$ and $\theta_{\max }=28.271^{\circ}$. The limiting indices were $-9 \leq h \leq 9,-12 \leq k \leq 13$, and $-27 \leq l \leq 27$. All hydrogen atoms attached to nitrogen and oxygen were freely refined and all other hydrogens were refined using the riding-model approximation. Convergence was reached on the full-matrix least-squares refinement of $F^{2}$. The $R^{1}$-factor is 0.0333 and $w R^{2}$ is 0.0792 . Figure 3.10 shows an ellipsoid drawing of $\mathbf{5}$. The ellipsoids are modeled at 50\% probability. 


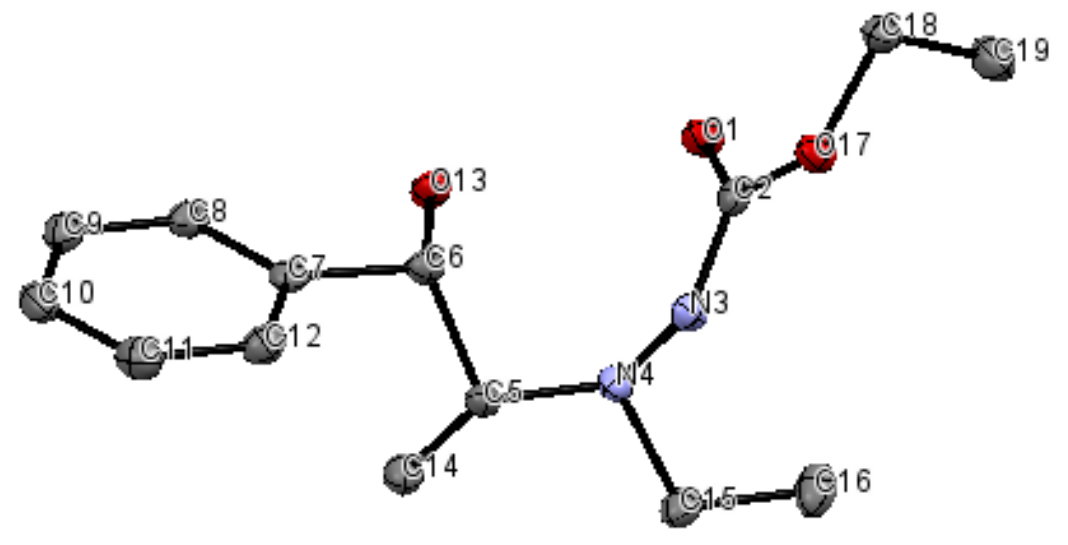

Figure 3.10 Ellipsoid depiction of $\mathbf{5}$.

The bond lengths, bond angles and torsion angles of the central oxadiazinanone ring as well as any carbon atoms attached to the ring are shown in Tables 3.17-19. The hydrogen bond lengths are given in Table 3.20. 
Table 3.17 Bond lengths between atoms in the asymmetric unit of 5 .

\begin{tabular}{ll}
\hline Atoms & Bond Length $(\AA)$ \\
\hline O1-C2 & $1.218(2)$ \\
C2-N3 & $1.350(2)$ \\
C2-O17 & $1.349(2)$ \\
N3-N4 & $1.419(2)$ \\
N4-C5 & $1.490(2)$ \\
N4-C15 & $1.479(2)$ \\
C5-C6 & $1.540(2)$ \\
C5-C14 & $1.529(2)$ \\
C6-C7 & $1.518(2)$ \\
C6-O13 & $1.433(2)$ \\
C7-C8 & $1.391(2)$ \\
C7-C12 & $1.399(2)$ \\
C8-C9 & $1.391(3)$ \\
C9-C10 & $1.385(3)$ \\
C10-C11 & $1.383(3)$ \\
C11-C12 & $1.389(3)$ \\
O13-H13 & $0.82(2)$ \\
C15-C16 & $1.517(2)$ \\
O17-C18 & $1.456(2)$ \\
C18-C19 & $1.505(2)$ \\
\hline
\end{tabular}


Table 3.18 Bond angles in 5.

\begin{tabular}{ll}
\hline Atoms & Angle $\mathbf{(}^{\mathbf{}} \mathbf{)}$ \\
\hline O1-C2-N3 & $126.0(2)$ \\
O1-C2-O17 & $124.9(1)$ \\
N3-C2-O17 & $109.0(1)$ \\
C2-N3-N4 & $118.3(1)$ \\
N3-N4-C5 & $110.1(1)$ \\
N3-N4-C15 & $108.0(1)$ \\
C5-N4-C15 & $112.3(1)$ \\
N4-C5-C6 & $108.4(1)$ \\
N4-C5-C14 & $109.9(1)$ \\
C6-C5-C14 & $111.4(1)$ \\
C5-C6-C7 & $111.5(1)$ \\
C5-C6-O13 & $110.5(1)$ \\
C7-C6-O13 & $110.1(1)$ \\
N4-C15-C16 & $112.1(1)$ \\
C2-O17-C18 & $116.0(1)$ \\
O17-C18-C19 & $110.5(1)$ \\
\hline
\end{tabular}


Table 3.19 Torsion angles in asymmetric unit of 5 .

\begin{tabular}{ll}
\hline Atoms & Torsion $\left(^{\mathbf{0}}\right)$ \\
\hline O1-C2-N3-N4 & $11.3(2)$ \\
O17-C2-N3-N4 & $-171.2(1)$ \\
O1-C2--O17-C18 & $-7.6(2)$ \\
N3-C2-O17-C18 & $174.9(1)$ \\
C2-N3-N4-C5 & $-115.8(2)$ \\
C2-N3-N4-C15 & $121.2(2)$ \\
N3-N4-C5-C6 & $56.6(2)$ \\
N3-N4-C5-C14 & $178.5(1)$ \\
C15-N4-C5-C6 & $177.0(1)$ \\
C15-N4-C5-C14 & $-61.1(2)$ \\
N3-N4-C15-C16 & $-59.0(2)$ \\
C5-N4-C15-C16 & $179.4(1)$ \\
N4-C5-C6-C7 & $179.1(1)$ \\
N4-C5-C6-O13 & $56.3(2)$ \\
C14-C5-C6-C7 & $58.0(2)$ \\
C14-C5-C6-O13 & $-64.7(2)$ \\
C2-O17-C18-C19 & $-80.1(2)$ \\
\hline
\end{tabular}

Table 3.20 Donor-Acceptor Hydrogen bond lengths in $\mathbf{5}$.

\begin{tabular}{lll}
\hline Donor-Acceptor & Distance, D-A (̊) & \multicolumn{1}{c}{ Bonding Motif } \\
\hline N3-O13 & 2.941 & Homoenantiomorph \\
O1-O13 & 2.893 & Intramolecular \\
\hline
\end{tabular}

\section{Crystallographic Details of 6}

In Figure 3.11 is a ChemDraw ${ }^{30}$ depiction of the product which resulted from the attempted synthesis of (5R,6S)-4-n-propyl-5-methyl-6-phenyl-3H-1,3,4-oxadiazinan-2one. 


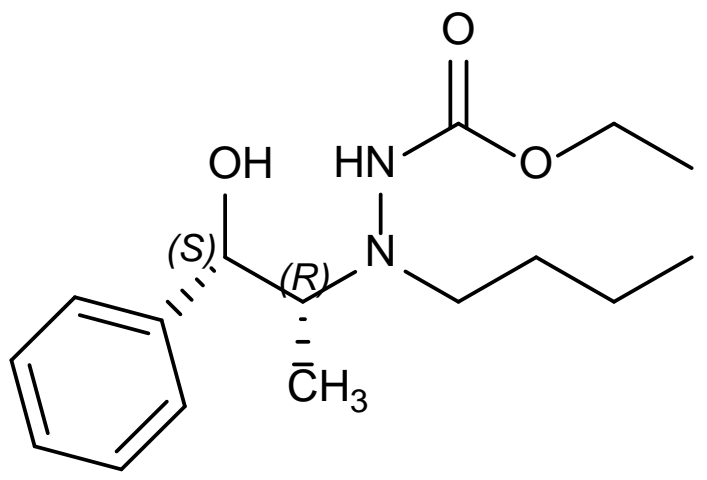

Figure 3.11 Line drawing of 6.

The dimensions of the crystal used for X-ray diffraction were $0.339 \mathrm{~mm}$ x 0.211 mm x $0.04 \mathrm{~mm}$. The sample was generally kept at 150(2) K. Superflip ${ }^{37}$ was used for solving the structure and Shelxl-2012 ${ }^{36}$ was used for structure refinement. The unit cell parameters were obtained from a least-squares refinement of 9971 unique reflections out of the 37783 reflections collected. The crystal is monoclinic and crystallizes in the $P 2_{1}$ space group. The cell lengths are: $a=9.6957(2) \AA, b=15.5239(4) \AA$, and $c=11.5672(3)$ A. Angle $\beta$ is $103.4220(10)^{\circ}$. The volume of the cell is $1693.48 \AA^{3}$ and $\rho=1.155 \mathrm{~g} / \mathrm{cm}^{3}$. $Z^{\prime}$ is 2 and $Z$ is 4 . Diffraction data were measured between $\theta_{\min }=2.23^{\circ}$ and $\theta_{\max }=$ $30.26^{\circ}$. The limiting indices were $-13 \leq h \leq 13,-21 \leq k \leq 21$, and $-15 \geq l \leq 15$. All hydrogen atoms attached to nitrogen and oxygen were freely refined and all other hydrogens were refined using the riding-model approximation. Convergence was reached on the full-matrix least-squares refinement of $F^{2}$. The $R^{1}$-factor is 0.0473 and $w R^{2}$ is 0.1274 Figure 3.12 shows an ellipsoid drawing of $\mathbf{6}$. The ellipsoids are modeled at $50 \%$ probability. 


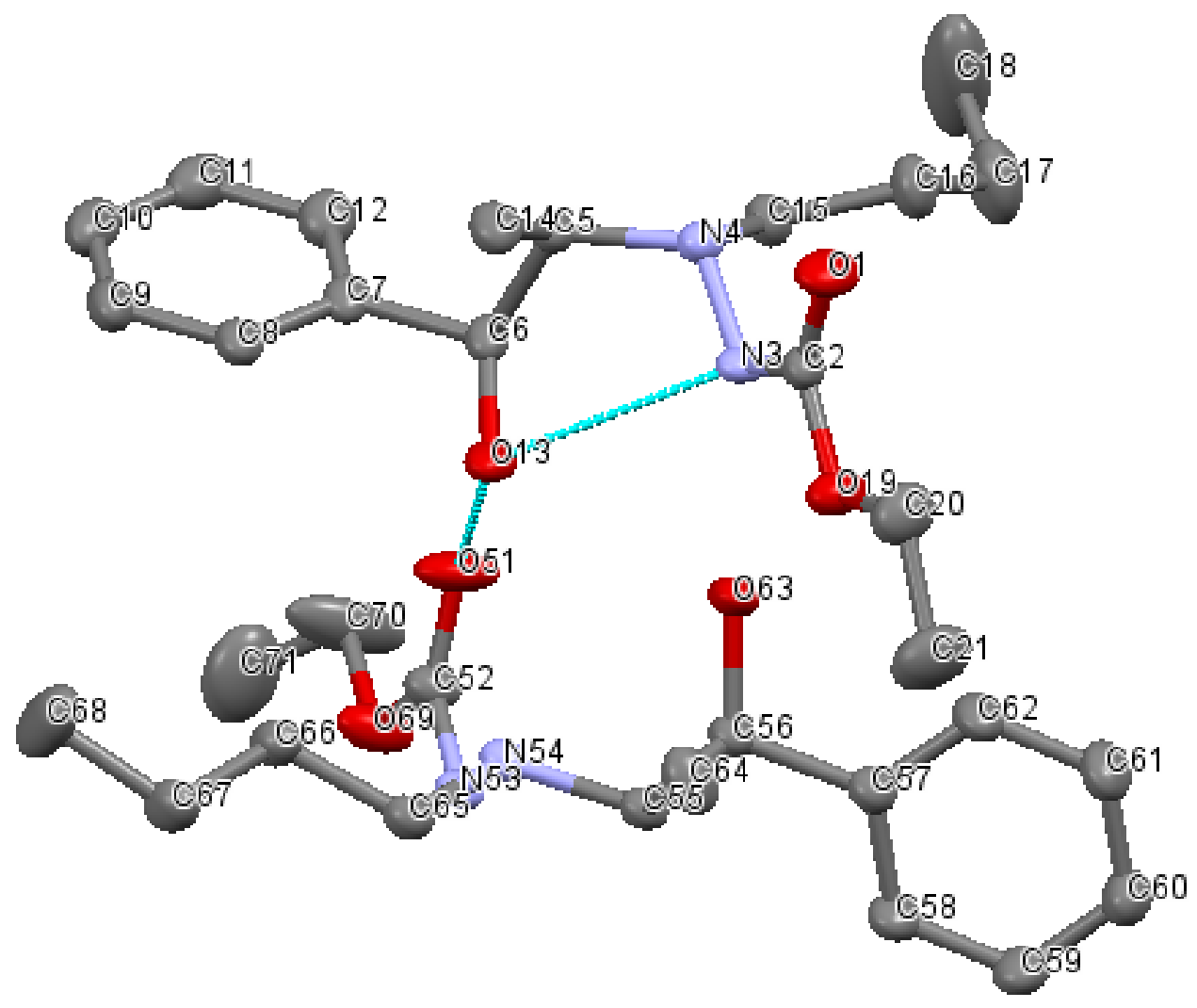

Figure 3.12 Ellipsoid depiction of $\mathbf{6}$.

The bond lengths, bond angles and torsion angles of the central oxadiazinanone ring as well as any carbon atoms attached to the ring are shown in Tables 3.21-24. The hydrogen bond lengths are shown in Table 3.24. 
Table 3.21 Bond lengths between atoms in the asymmetric unit of $\mathbf{6}$.

\begin{tabular}{ll||lc}
\hline Atoms-Molecule 1 & Length $(\AA)$ & Atoms-Molecule 2 & Length $(\AA)$ \\
\hline O1-C2 & $1.217(3)$ & O51-C52 & $1.215(3)$ \\
C2-N3 & $1.343(3)$ & C52-N53 & $1.335(3)$ \\
C2-O19 & $1.337(3)$ & C52-O69 & $1.342(3)$ \\
N3-N4 & $1.404(3)$ & N53-N54 & $1.418(3)$ \\
N4-C5 & $1.484(3)$ & N54-C55 & $1.491(3)$ \\
N4-C15 & $1.468(3)$ & N54-C65 & $1.477(3)$ \\
C5-C6 & $1.549(3)$ & C55-C56 & $1.540(3)$ \\
C5-C14 & $1.517(3)$ & C55-C64 & $1.529(4)$ \\
C6-C7 & $1.518(3)$ & C56-C57 & $1.510(3)$ \\
C6-O13 & $1.423(3)$ & C56-O63 & $1.423(2)$ \\
C15-C16 & $1.530(4)$ & C65-C66 & $1.521(3)$ \\
C16-C17 & $1.513(4)$ & C66-C67 & $1.519(4)$ \\
C17-18 & $1.471(6)$ & C67-C68 & $1.514(4)$ \\
O19-C20 & $1.442(4)$ & O69-C70 & $1.457(5)$ \\
C20-C21 & $1.485(4)$ & C70-C71 & $1.477(8)$ \\
\hline
\end{tabular}

Table 3.22 Bond angles in 6.

\begin{tabular}{ll||lc}
\hline \multicolumn{1}{c}{ Atoms-Molecule 1 } & Angle $\left(^{\circ}\right)$ & Atoms-Molecule 2 & Angle $\left(^{\circ}\right)$ \\
\hline O1-C2-N3 & $126.1(2)$ & O51-C52-N53 & $125.5(2)$ \\
O1-C2-O19 & $124.8(2)$ & O51-C52-O69 & $123.7(2)$ \\
N3-C2-O19 & $109.1(2)$ & N53-C52-O69 & $110.7(2)$ \\
C2-N3-N4 & $120.3(2)$ & C52-N53-N54 & $117.6(2)$ \\
N3-N4-C5 & $112.6(2)$ & N53-N54-C55 & $110.4(2)$ \\
N3-N4-C15 & $109.2(2)$ & N53-N54-C65 & $108.0(2)$ \\
C5-N4-C15 & $113.9(2)$ & C55-N54-C65 & $114.8(2)$ \\
N4-C5-C6 & $114.5(2)$ & N54-C55-C56 & $108.2(2)$ \\
N4-C5-C14 & $109.7(2)$ & N54-C55-C64 & $110.1(2)$ \\
C6-C5-C14 & $112.1(2)$ & C56-C55-C64 & $110.3(2)$ \\
C5-C6-C7 & $111.4(2)$ & C55-C56-C57 & $111.4(2)$ \\
C5-C6-O13 & $108.1(2)$ & C55-C56-O63 & $111.6(2)$ \\
C7-C6-O13 & $112.6(2)$ & C57-C56-O63 & $110.1(2)$ \\
N4-C15-C16 & $110.3(2)$ & N54-C65-C66 & $111.6(2)$ \\
C15-C16-C17 & $113.3(2)$ & C65-C66-C67 & $112.4(2)$ \\
C16-C17-C18 & $114.2(4)$ & C66-C67-C68 & $112.3(3)$ \\
C2-O19-C20 & $115.9(2)$ & C52-O69-C70 & $115.4(3)$ \\
O19-C20-C21 & $107.1(3)$ & O69-C70-C71 & $109.0(4)$ \\
\hline
\end{tabular}


Table 3.23 Torsion angles in asymmetric unit of $\mathbf{6}$

\begin{tabular}{ll||ll}
\hline Atoms-Molecule 1 & Torsion ( ${ }^{\circ}$ & Atoms-Molecule 2 & Torsion ( ${ }^{\circ}$ \\
\hline O1-C2-N3-N4 & $1.2(4)$ & O51-C52-N53-N54 & $9.2(3)$ \\
O19-C2-N3-N4 & $-178.6(2)$ & O69-C52-N53-N54 & $-171.6(2)$ \\
O1-C2-O19-C20 & $0.3(3)$ & O51-C52-O69-C70 & $1.7(4)$ \\
N3-C2-O19-C20 & $-179.9(2)$ & N53-C52-O69-C70 & $-177.6(3)$ \\
C2-N3-N4-C5 & $-113.7(2)$ & C52-N53-N54-C55 & $-115.4(2)$ \\
C2-N3-N4-C15 & $118.7(2)$ & C52-N53-N54-C65 & $118.4(2)$ \\
N3-N4-C5-C6 & $-60.3(2)$ & N53-N54-C55-C56 & $60.5(2)$ \\
N3-N4-C5-C14 & $66.8(2)$ & N53-N54-C55-C64 & $-178.9(2)$ \\
C15-N4-C5-C6 & $64.8(2)$ & C65-N54-C55-C56 & $-177.2(2)$ \\
& $-168.1(2)$ & C65-N54-C55-C64 & $-56.5(2)$ \\
N3-N4-C15-C16 & $-72.3(2)$ & N53-N54-C65-C66 & $-68.9(2)$ \\
C5-N4-C15-C16 & $160.9(2)$ & C55-N54-C65-C66 & $167.5(2)$ \\
N4-C5-C6-C7 & $-162.4(2)$ & N54-C55-C56-C57 & $-173.7(2)$ \\
N4-C5-C6-O13 & $73.4(2)$ & N54-C55-C56-O63 & $62.7(2)$ \\
C14-C5-C6-C7 & $71.8(2)$ & C64-C55-C56-C57 & $65.8(2)$ \\
C14-C5-C6-O13 & $-52.4(2)$ & C64-C55-C56-O63 & $-57.7(2)$ \\
C5-C6-C7-C8 & $-95.5(3)$ & C55-C56-C57-C58 & $69.6(3)$ \\
C5-C6-C7-C12 & $85.2(3)$ & C55-C56-C57-C62 & $-111.3(2)$ \\
O13-C6-C7-C8 & $26.1(3)$ & O63-C56-C57-C58 & $-166.0(2)$ \\
O13-C6-C7-C12 & $-153.2(2)$ & O63-C56-C57-C62 & $13.1(3)$ \\
N4-C15-C16-C17 & $171.8(2)$ & N54-C65-C66-C67 & $175.4(2)$ \\
C15-C16-C17-C18 & $64.8(4)$ & C65-C66-C67-C68 & $172.3(3)$ \\
C2-O19-C20-C21 & $-179.0(2)$ & C52-O69-C70-C71 & $-159.2(3)$ \\
\hline
\end{tabular}

Table 3.24 Donor-Acceptor Hydrogen bond lengths in $\mathbf{6}$.

\begin{tabular}{lll}
\hline Donor-Acceptor & Distance, D-A (A) & Bonding Motif \\
\hline O13-N3 & 2.883 & Intramolecular \\
O13-O51 & 2.908 & Heteroenantiomorph \\
\hline
\end{tabular}




\section{Summary of Crystallographic Details of Crystals Studied in This Research}

Tables 3.25 and 3.26 summarize the crystallographic details in this research.

Table 3.25 Summary data for crystallographic details of the oxadiazinanone crystals in this thesis.

\begin{tabular}{|c|c|c|c|c|}
\hline Compound & 1 & 2 & 3 & 4 \\
\hline $\mathrm{N}_{4}$ Substituent/type & cyp/pure $(5 R, 6 S)$ & $\begin{array}{l}\text { cyp } \\
\text { /racemic }\end{array}$ & $\begin{array}{l}(5 S, 6 R) \text { сур/ } \\
(5 R, 6 S) \text { iso }\end{array}$ & $\begin{array}{l}\text { (5S,6R(сур)/ } \\
(5 R, 6 S) \text { iso }\end{array}$ \\
\hline Formula & $\mathrm{C}_{15} \mathrm{H}_{2} \mathrm{~N}_{2} \mathrm{O}_{2}$ & $\mathrm{C}_{15} \mathrm{H}_{20} \mathrm{~N}_{2} \mathrm{O}_{2}$ & $\mathrm{C}_{28} \mathrm{H}_{38} \mathrm{~N}_{4} \mathrm{O}_{4}$ & $\mathrm{C}_{31} \mathrm{H}_{42} \mathrm{~N}_{4} \mathrm{O}_{4}$ \\
\hline Crystal Setting & orthorhombic & monoclinic & monoclinic & Monoclinic \\
\hline $\begin{array}{l}\text { Space Group } \\
\text { Crystal Size } \\
\left(\mathrm{mm}^{3}\right)\end{array}$ & $\begin{array}{l}P 2_{1} 2_{1} 2_{1} \\
0.360 \times 0.270 \times \\
0.210\end{array}$ & $\begin{array}{l}P 2_{1} / c \\
0.195 \times 0.138 \times \\
0.137\end{array}$ & $\begin{array}{l}P 2_{1} \\
0.449 \times 0.211 \times \\
0.102\end{array}$ & $\begin{array}{l}P 2_{1} \\
0.330 \times 0.120 \times \\
0.100\end{array}$ \\
\hline$a(\AA)$ & $8.4171(3)$ & $9.1808(3)$ & 10.4323(3) & 8.8830(3) \\
\hline$b(\AA)$ & $9.0618(4)$ & $15.7560(5)$ & $8.7068(2)$ & $16.915(5)$ \\
\hline$c(\AA)$ & $36.7815(13)$ & $9.7293(3)$ & $14.6212(4)$ & $10.0167(3)$ \\
\hline$\alpha\left(^{\circ}\right)$ & 90 & 90 & 90 & 90 \\
\hline$\beta\left(^{\circ}\right)$ & 90 & $106.132(2)$ & $94.1200(10)$ & $106.432(2)$ \\
\hline$\gamma\left({ }^{\circ}\right)$ & 90 & 90 & 90 & 90 \\
\hline$V \AA^{3}$ & 2805.47 & 1351.95 & 1324.64 & $1443.55(8)$ \\
\hline$Z ; Z^{\prime}$ & $2 ; 8$ & $1 ; 4$ & $1 ; 2$ & $2 ; 8$ \\
\hline Density & 1.233 & 1.279 & 1.240 & 1.230 \\
\hline Temperature & $80(2) \mathrm{K}$ & 293(2) K & 293(2) K & $100(2) \mathrm{K}$ \\
\hline Total \# reflections & 17571 & 19297 & 52929 & 53541 \\
\hline$R_{\text {-factor }}^{1}$ & 0.0432 & 0.0381 & 0.0427 & 0.0455 \\
\hline$\omega R^{2}$ & 0.1029 & 0.0926 & 0.1098 & 0.1126 \\
\hline Unique reflections & 4581 & 2460 & 8339 & 7852 \\
\hline$S$ & 1.160 & 1.034 & 1.036 & 1.019 \\
\hline
\end{tabular}


Table 3.26 Summary of crystallographic details of crystals studied in this research which were not oxadiazinanones.

\begin{tabular}{lll}
\hline \multicolumn{1}{c}{ Compound } & \multicolumn{1}{c}{$\mathbf{5}$} & \multicolumn{1}{c}{$\mathbf{6}$} \\
\hline Formula & $\mathrm{C}_{14} \mathrm{H}_{22} \mathrm{~N}_{2} \mathrm{O}_{3}$ & $\mathrm{C}_{16} \mathrm{H}_{26} \mathrm{~N}_{2} \mathrm{O}_{3}$ \\
Crystal Setting & orthorhombic & monoclinic \\
Space Group & $P 2_{1} 2_{1} 2_{1}$ & $P 2_{1}$ \\
Crystal Size $\left(\mathrm{mm}^{3}\right)$ & $0.248 \times 0.201 \times 0.061$ & $0.339 \times 0.211 \times 0.04$ \\
$a(\AA)$ & $7.0046(8)$ & $9.6957(2)$ \\
$b(\AA)$ & $10.0479(11)$ & $15.5239(4)$ \\
$c(\AA)$ & $20.587(2)$ & $11.5672(3)$ \\
$\alpha\left(^{\circ}\right)$ & 90 & 90 \\
$\beta\left(^{\circ}\right)$ & 90 & $103.4220(10)$ \\
$\gamma\left(^{\circ}\right)$ & 90 & 90 \\
$V \AA^{3}$ & 1448.94 & 1693.48 \\
$Z ; Z^{\prime}$ & $1 ; 4$ & $2 ; 4$ \\
density g/cm & 3 \\
Temperature & 1.221 & 1.155 \\
Total \# reflections & $100(2) \mathrm{K}$ & $150(2) \mathrm{K}$ \\
$R^{1}$-factor & 15264 & 37783 \\
$\omega R^{2}$ & 0.0333 & 0.0473 \\
Unique reflections $_{S}$ & 0.0792 & 0.1274 \\
& 5033 & 9971 \\
& 1.038 & 1.02
\end{tabular}




\section{CHAPTER IV}

\section{DISCUSSION OF CRYSTAL STRUCTURES}

In order to better understand the similarities and differences in the oxadiazinanone compounds studied during this research, it would be helpful to have a summary of some

of the relevant crystallographic details. ${ }^{24,26,38}$ The crystal packing and hydrogen-bonding motifs will be compared and contrasted in an attempt to understand what causes some oxadiazinanones to fail to fractionally crystallize and others to succeed. In addition, each of the new crystal structures found during this research will be discussed.

\section{Summary of the Formation of Quasiracemic Crystals and Some Key Crystallographic Data Pertaining to the Oxadiazinanones Studied}

Tables 4.1-4 summarize the information for each of the enantiomers studied.

Table 4.1 gives the results for each attempt made to isolate a quasiracemic crystal. If a quasiracemic crystal formed (failure of fractional crystallization), the term "quasi," highlighted in blue, can be seen. If a combination was not attempted, the designation N/A is given. When enantiomers of an oxadiazinanone form a racemic crystal, it is highlighted in peach. In cases of fractional crystallization, the data show which quasienantiomer was found (based on the $\mathrm{N}_{4}$ substituent). Tables 4.2-4.3 give crystallographic details for pure structures, racemic structures and quasiracemic structures of oxadiazinanones, respectively. In Tables 4.2-4.4 the hydrogen-bonding motif refers to whether the 
hydrogen bonds occur between structures with the same chirality (enantiomorphs) or the opposite chirality. The bonds between molecules with the same chirality are called homo enantiomorphic bonds. The bonds between molecules with different chirality are called hetero enantiomorphic bonds.

Table 4.1 Fractional crystallization results of the combination of $(5 R, 6 S)$ oxadiazinanones and $(5 S, 6 R)$ oxadiazinanones.

\begin{tabular}{|c|c|c|c|c|c|c|}
\hline & & \multicolumn{5}{|c|}{$\mathrm{N}_{4}$ Substituent on $(5 R, 6 S)$ Oxadiazinanone } \\
\hline & & cyclohexyl & $\begin{array}{l}\text { cyclo- } \\
\text { pentyl }\end{array}$ & isopropyl & n-propyl & methyl \\
\hline & cyclohexyl & racemate $^{2}$ & quasi & quasi $^{2}$ & cyclohexyl & methyl \\
\hline \multirow{4}{*}{$\begin{array}{c}\mathrm{N}_{4} \\
\text { Substituent } \\
\text { on }(5 S, 6 R) \\
\text { Oxadiazina- } \\
\text { none }\end{array}$} & cyclopentyl & quasi & racemate & quasi & cyclopentyl & methyl \\
\hline & isopropyl & quasi $^{2}$ & $\mathrm{~N} / \mathrm{A}^{3}$ & racemate $^{1}$ & isopropyl & methyl \\
\hline & n-propyl & $\mathrm{N} / \mathrm{A}^{3}$ & $\mathrm{~N} / \mathrm{A}^{3}$ & $\mathrm{~N} / \mathrm{A}^{3}$ & racemate $^{1}$ & $\mathrm{~N} / \mathrm{A}^{3}$ \\
\hline & methyl & methyl & $\mathrm{N} / \mathrm{A}^{3}$ & $\mathrm{~N} / \mathrm{A}^{3}$ & $\mathrm{~N} / \mathrm{A}^{3}$ & racemate $^{1}$ \\
\hline
\end{tabular}

${ }^{1}$ Racemates are assumed to form. However the cyclopentyl racemate was the only racemate attempted in this research.

${ }^{2}$ Kate Edler crystallized and reported on these crystals. ${ }^{26}$

${ }^{3} \mathrm{~N} / \mathrm{A}$ indicates the combination was not attempted or crystals were not obtained.

Table 4.2 Crystallographic details of enantiomerically pure compounds.

\begin{tabular}{lcccc}
\hline $\begin{array}{c}\mathbf{N}_{\mathbf{4}} \\
\text { substituent }\end{array}$ & $\begin{array}{c}\text { Space } \\
\text { Group }\end{array}$ & $\begin{array}{c}\text { Crystal } \\
\text { Setting }\end{array}$ & $\mathbf{Z} ; \boldsymbol{Z}^{\prime}$ & $\begin{array}{c}\text { No. of H-Bonds; Motif } \\
\text { (Asymmetric Unit) }\end{array}$ \\
\hline cyclohexyl (7) & $P 2_{1}{ }_{1}{ }_{1}$ & orthorhombic & $16 ; 4$ & 4 ; homo-enantiomorph \\
cyclopentyl (1) & $P 2_{1}{ }_{1}{ }_{1}$ & orthorhombic & $8 ; 2$ & 2 ; homo-enantiomorph \\
isopropyl (9) & $P 2_{1} 2_{1} 2_{1}$ & orthorhombic & $8 ; 2$ & 2 ; homo-enantiomorph \\
n-propyl (13) & $P 2_{1}$ & monoclinic & $4 ; 2$ & 4 ; homo-enantiomorph \\
methyl (12) & $P 2_{1}$ & monoclinic & $2 ; 1$ & 2; homo-enantiomorph \\
\hline
\end{tabular}


Table 4.3 Crystallographic details of racemic compounds.

\begin{tabular}{lcccc}
\hline $\mathbf{N}_{\mathbf{4}}$ substituent & $\begin{array}{c}\text { Space } \\
\text { Group }\end{array}$ & $\begin{array}{c}\text { Crystal } \\
\text { Setting }\end{array}$ & Z; $Z$, & $\begin{array}{c}\text { No. of H-Bonds; Motif } \\
\text { (Asymmetric Unit) }\end{array}$ \\
\hline isopropyl (11) & $C 2 / c$ & monoclinic & $8 ; 1$ & 2; hetero-enantiomorph \\
cyclopentyl (2) & $P 2_{1} / \mathrm{c}$ & monoclinic & $4 ; 1$ & 2; hetero-enantiomorph \\
cyclohexyl(10) & $P-1$ & triclinic & $4 ; 2$ & 4; hetero-enantiomorph \\
\hline
\end{tabular}

Table 4.4 Crystallographic details of quasiracemic compounds.

\begin{tabular}{lcccc}
\hline \multicolumn{1}{c}{ N$_{\text {4 }}$ substituents } & $\begin{array}{c}\text { Space } \\
\text { Group }\end{array}$ & $\begin{array}{c}\text { Crystal } \\
\text { Setting }\end{array}$ & Z; Z, & $\begin{array}{c}\text { No. of H-Bonds; Motif } \\
\text { (Asymmetric Unit) }\end{array}$ \\
\hline isopropyl/cyclopentyl (3) & $P 2_{1}$ & monoclinic & $4 ; 2$ & 4; hetero-enantiomorph \\
isopropyl/cyclohexyl (8) & $P 2_{1}$ & monoclinic & $4 ; 2$ & 4; hetero-enantiomorph \\
cyclohexyl/cyclopentyl (4) & $P 2_{1}$ & monoclinic & $4 ; 2$ & 4; hetero-enantiomorph \\
\hline
\end{tabular}

Most of the oxadiazinanones studied have $Z$ ’ > 1 . Compound 7 has $Z$ ’ of 4 . Table 4.5 shows that of the more than 600,000 structures in the Cambridge Structural Database a $Z^{\prime} \leq 1$ occurs in about $88 \%$ of the crystals. A $Z^{\prime}$ of 2 is much less common, and $Z^{\prime}$ of 4 is encountered less than $0.4 \%$ of the time. ${ }^{39}$ These unusually high $Z$ ' values may provide insight into why some of the compounds form quasiracemic crystals and others do not. 
Table 4.5 The occurrence frequency of structures between $Z$ ' ranges in the Cambridge Structural Database in 2013. ${ }^{39}$

\begin{tabular}{cc}
\hline Range of $Z^{\prime}$ & \% \\
\hline $0 \leq Z^{\prime} \leq 1$ & $88.2 \%$ \\
$1<Z^{\prime} \leq 2$ & $10.5 \%$ \\
$2<Z^{\prime} \leq 3$ & $0.6 \%$ \\
$3<Z^{\prime} \leq 4$ & $0.4 \%$ \\
$4<Z^{\prime}$ & $0.1 \%$ \\
\hline
\end{tabular}

In each quasiracemic crystal the $Z$ ' is less than or equal to the $Z$ ' of the component quasienantiomers. This is especially notable in the cyclopentyl/cyclohexyl quasiracemate. The cyclopentyl quasienantiomer, $\mathbf{1}$, has a $Z$ ’ of 2, and the cyclohexyl quasienantiomer, $\mathbf{7}$, has a $Z$ ' of 4 . After mixing the quasienantiomers together and crystallizing them, the resulting quasiracemate, 3 , has a $Z$ ' of 2 . Since this $Z$ ' of 2 includes the two quasienantiomers, it would be comparable to a Z' of 1 in a racemate crystal. When the methyl quasienantiomer is combined with any other quasienantiomer, the methyl always fractionally crystallizes.

Kitaigorodskii’s close-packing principle states that reduction of volume and increase in density are major factors in determining the ease with which molecules pack in the solid state. ${ }^{40}$ Brock and Dunitz noted that structures with large $Z$ ' values are associated with packing conflicts. Packing conflicts make it difficult to crystallize some compounds. ${ }^{34}$ Although $Z$ ' for $\mathbf{7}$ is 4 , when it co-crystallizes with $\mathbf{1}$ or $\mathbf{9}$ the resulting quasiracemic crystal has Z' of 2 which is comparable to a Z' of 1 in a racemate. This may indicate reduction in packing conflict with the quasiracemate crystal as compared to that 
of either of the pure oxadiazinanone enantiomers making up the racemates.

According to Braun et al., Z' of 1 is commonly found in racemic crystals, and he indicated that inversion symmetry is a probable reason. ${ }^{41}$ In a study by Kelley et al. it was found that inversion symmetry is very favorable for crystal packing. Inversion symmetry generally allows the molecules in the crystal to pack in less space, a factor which favors crystallization according to the close-packing principle. ${ }^{1}$ Brook et al. observed that the majority of crystallizations from racemic solutions lead to racemic crystals. ${ }^{42}$ The most common space groups, $P 1, P 2_{1} / c, C 2 / c$ and $P b c a$, are racemic and these four space groups account for approximately two-thirds of all organic crystals. ${ }^{42}$ Even though quasiracemates do not form true inversion centers, there are pseudo-inversion centers. The presence of these pseudo-inversion centers may make the quasiracemic crystal more stable than either of the quasienantiomeric crystals. This would explain why 3, 4, and 8 form quasiracemic crystals. It seems the value of $Z$ ' of the constituents has some impact on $Z$ ' of the quasiracemic crystal. The pseudo-inversion possible with quasiracemic crystals also seems to reduce packing conflicts. If packing conflicts can be alleviated by the formation of a quasiracemic crystal, the quasiracemic crystal is more likely to crystallize than either of the oxadiazinanone quasienantiomers.

Another observation can be made from the data in Table 4.1. When the isopropyl derivative and the $n$-propyl derivative were mixed, the isopropyl derivative crystallized. When the cyclohexyl and methyl derivatives were mixed, the methyl derivative crystallized. Finally, when the cyclohexyl and the $n$-propyl derivatives were mixed, the cyclohexyl was crystallized. The $n$-propyl group is more flexible than the isopropyl group which may help to make it more soluble in the ethyl acetate used for the crystallization 
process. In Figure 4.1, the connecting motif from $\mathrm{N} 4$ to the carbon on the $\mathrm{N}_{4}$ substituent is highlighted in blue. The cyclohexyl and cyclopentyl substituents have a connection with $\mathrm{N}_{4}$ similar to that of isopropyl, and they crystallize more readily than the oxadiazinanone with the $n$-propyl substituent which indicates that they may be more stable and able to crystallize more easily. In combinations which included a quasienantiomer with methyl as the $\mathrm{N}_{4}$ substituent, the quasienantiomer with an $\mathrm{N}_{4}$ of methyl always crystallized first. Methyl is the smallest $\mathrm{N}_{4}$ substituent and will have the least flexibility possible.

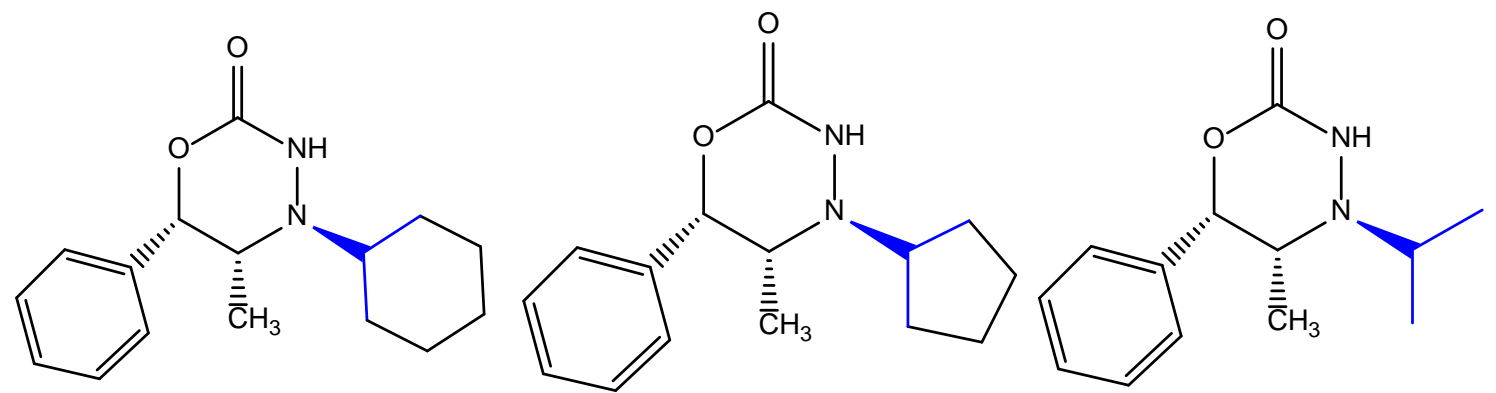

Figure 4.1 Line drawings of 9, 10, and 12 .

The principle that says crystallization favors the reduction of packing conflicts can be observed in the results of the crystallization studies shown in Tables 4.1-4.4. There seems to be indications that flexibility in a compound's bonds impacts crystallization. When $\mathbf{1 3}$, the $n$-propyl derivative, was mixed with a quasienantiomer, $\mathbf{1 3}$ appeared to remain in solution. Each time 12, the methyl derivative was mixed with a quasienantiomer, 12 was the crystal which was isolated. From this it seems that when methyl is the $\mathrm{N}_{4}$ substituent, it can be crystallized with few packing conflicts. On the other hand, when $n$-propyl is the $\mathrm{N}_{4}$ substituent it is less likely to crystallize. This could 
be due to the flexibility in its structure which can create packing conflicts.

It is always important to note that only one crystal from each product was analyzed by X-ray diffraction. If one determines the existence of a quasiracemic crystal, the certainty is that the combination of quasienantiomers can form a quasiracemate. In the case of no quasiracemic crystal being found, one can only say for certain that a quasiracemate was not found.

Hydrogen bonding remains a significant driving force in the determination of the shapes of crystal. ${ }^{40}$ The most acidic hydrogen atoms will bond with the most readily available hydrogen acceptors. In the case of the oxadiazinanones, the hydrogen bonding always has the form, $R_{2}^{2}(8) .{ }^{14}$ This means the hydrogen bonds form an eight-member ring with two hydrogen bond donors and two acceptors. The hydrogen bond occurs between the carbonyl groups and the amine hydrogens on the molecules in the crystal. According to the literature, ${ }^{38}$ the bond lengths between the C3-N3 carbons in oxadiazinanones which are unsubstituted at the N3 position (the case for all the oxadiazinanones studied in this research) is shorter than those which are substituted. The C3-N3 bond lengths in oxadiazinanones unsubstituted at N3 are in the range 1.389-1.418 $\AA$. Two structures substituted at N3 have C3-N3 bond lengths of 1.337(2) $\AA$ and 1.342(2) $\AA .{ }^{39}$ This indicates the presence of greater double bond character for this bond. This double bond character could play a role in the strong preference these compounds have for hydrogenbonding. It is worth noting that hydrogen bonding can occur between the same enantiomorphs, referred to as a homo-enantiomorph motif, or different ones, a heteroenantiomorph motif. Tables 4.2-4.4 indicate the bonding motif for each crystal studied. Another driving force for a particular type of crystal packing is the minimization 
of electrostatic energy. Although this is very important in ionic crystals, it also is true in molecular crystals. ${ }^{40}$ The localized charges on the atoms are small, but remain important in the packing of the crystals. Minimizing these forces happens when positive and negative charges are optimized. As described by Brock, this is much like having bumps against hollows, donors against acceptors, positive ends of dipoles against negative ends. ${ }^{40}$ This helps to increase density and minimize volume, implying reduced packing conflicts. Hydrogen-bonding which is prevalent in oxadiazinanone crystals can help to minimize electrostatic energy.

Springuel et al. summarized the formation of co-crystals by concluding that the formation of co-crystals is a summation of molecular geometry, steric hindrance and hydrogen-bonding. ${ }^{43}$ This appears to be the case in the structures of this thesis.

\section{Analysis of (5R,6S)-4-Cyclopentyl-5-methyl-6-phenyl-2H-1,3,4-oxadiazinan-2-one, 1}

Compound 1 packs in space group $P 2{ }_{1} 2{ }_{1} 2_{1}$. This non-centrosymmetric group has three screw axes orthogonal to each other with no other symmetry elements. Because there is no other symmetry, the two molecules are not crystallographically identical. This could be due to conformational differences in the two molecules or flexibility in the hydrogen-bonding. ${ }^{44}$ There are two molecules in the asymmetric unit. The packing diagram is shown in Figure 4.2. 


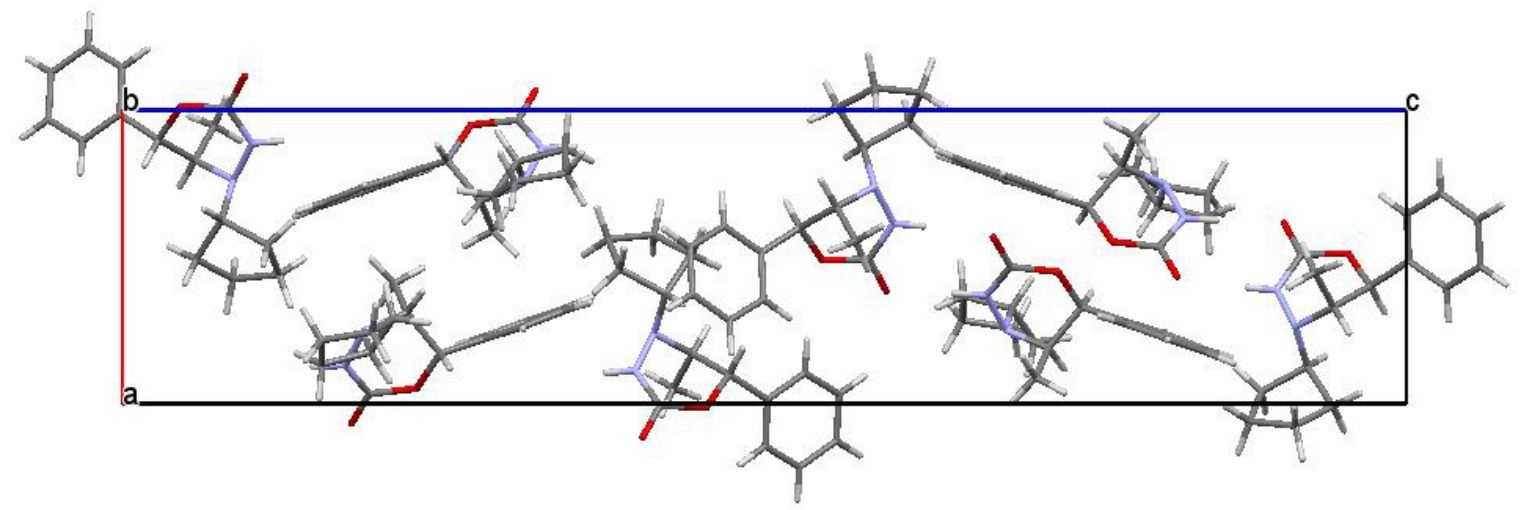

Figure 4.2 Packing diagram of 1.

The data from an X-ray diffractometer must be converted to a set of coordinates which describe the crystal in three-dimensional space. Once this data has been obtained, Mercury a software package developed by the Cambridge Crystallographic Data Centre, can create images of the crystals. This data can be used to analyze crystal structures including bond distances, torsion angles, hydrogen bonding and symmetry. One reason for having more than one molecule in the asymmetric unit of a crystal is that the molecules have different conformations. ${ }^{41}$ To determine whether this is the case for $\mathbf{1}$, an overlay was done using Mercury. ${ }^{45}$ In Figure 4.3 is a wireframe depiction of $\mathbf{1}$. The three atoms in the central oxadiazinanone ring marked in yellow were chosen as the atoms over which a least squares fit was calculated. 


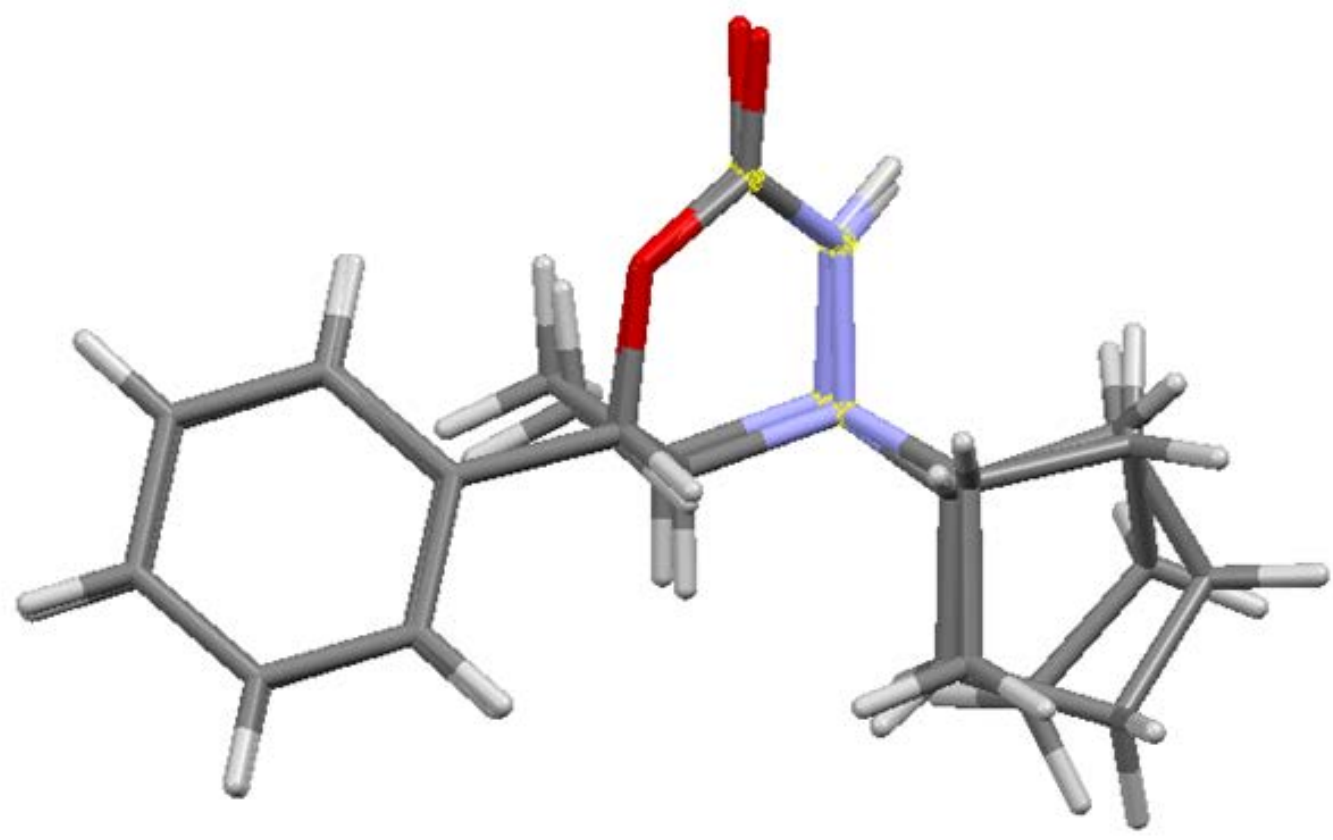

Figure 4.3 Overlay of the two molecules in the asymmetric unit of $\mathbf{1}$.

When an overlay is calculated in Mercury, ${ }^{45}$ the closest fit will be found in the atoms chosen for the least squares calculation, and the farther from these atoms one goes, the greater the differences will appear. Figure 4.3 shows that these two molecules differ the most in their structure where the cyclopentyl group is located. This could be the source of the unexpected asymmetry. Even when the overlay is calculated using atoms in the cyclopentyl substituent, there is still a difference in the conformation of the cyclopentyl group.

When two molecules are present in the asymmetric unit of pure enantiomers, hydrogen bonding can allow some flexing of the bonds. This creates some slight differences in the two molecules. This flexing creates a situation which distorts the inversion centers. $^{1}$ 


\section{Analysis of the Racemate of 4-Cyclopentyl-5-methyl-6-phenyl-2H-1,3,4-oxadiazinan- 2-one, 2}

Compound 2 crystallizes in the non-chiral, centrosymmetric space group $P 2_{1} / c$, the most common space group. This space group comprises over one-third of the crystals in the Cambridge Structural Database. ${ }^{46}$ Because the crystal is a racemate it does not have optical activity. Although two different enantiomers are present in the structure, there is only one molecule in the asymmetric unit $\left(Z^{\prime}=1\right)$ because the enantiomers, by definition, are mirror images of each other. That means that if there is one enantiomer present, the other can be obtained by a symmetry operation (inversion). The unit cell has two glide planes, two 2-fold screw axes and an inversion center. The packing diagram in Figure 4.4 shows the 4 structures in the unit cell.

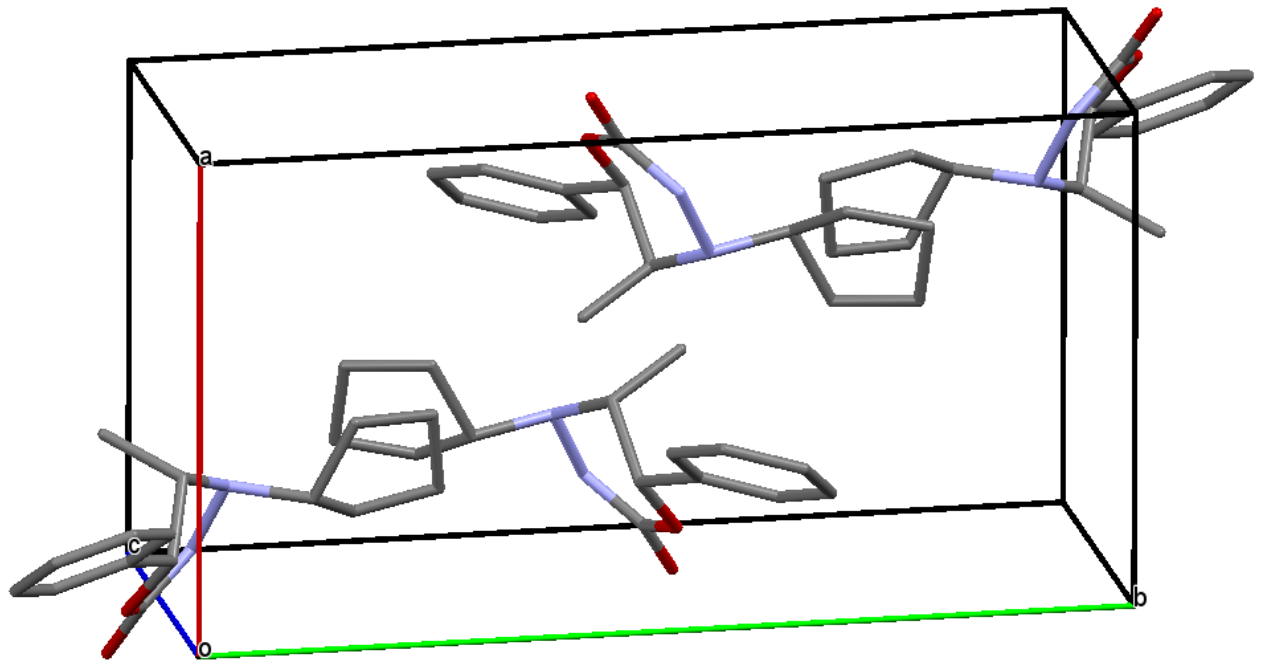

Figure 4.4 Packing diagram of 2.

In the racemate, 2, hydrogen-bonding is observed between different enantiomorphs in the crystal. This differs from the bonding that occurs in $\mathbf{1 1}$ (see Table 
4.3) where the hydrogen bonding occurs between the same enantiomorphs even though it is also a racemic crystal.

Analysis of the Quasiracemate of (5R,6S)-5-Methyl-4-cyclopentyl-6-phenyl-2H-1,3,4oxadiazinan-2-one and (5S,6R)-4-Isopropyl-5-methyl-6-phenyl-2H-1,3,4oxadiazinan-2-one, 3

Compound 3 packs in the non-centrosymmetric space group $P 2_{1}$ and has one symmetry element, a screw axis. The hydrogen-bonding motif is hetero-enantiomorphic. There are 2 molecules in the asymmetric unit (see Figures 3.9 and 10). The packing diagram can be seen in Figure 4.5.

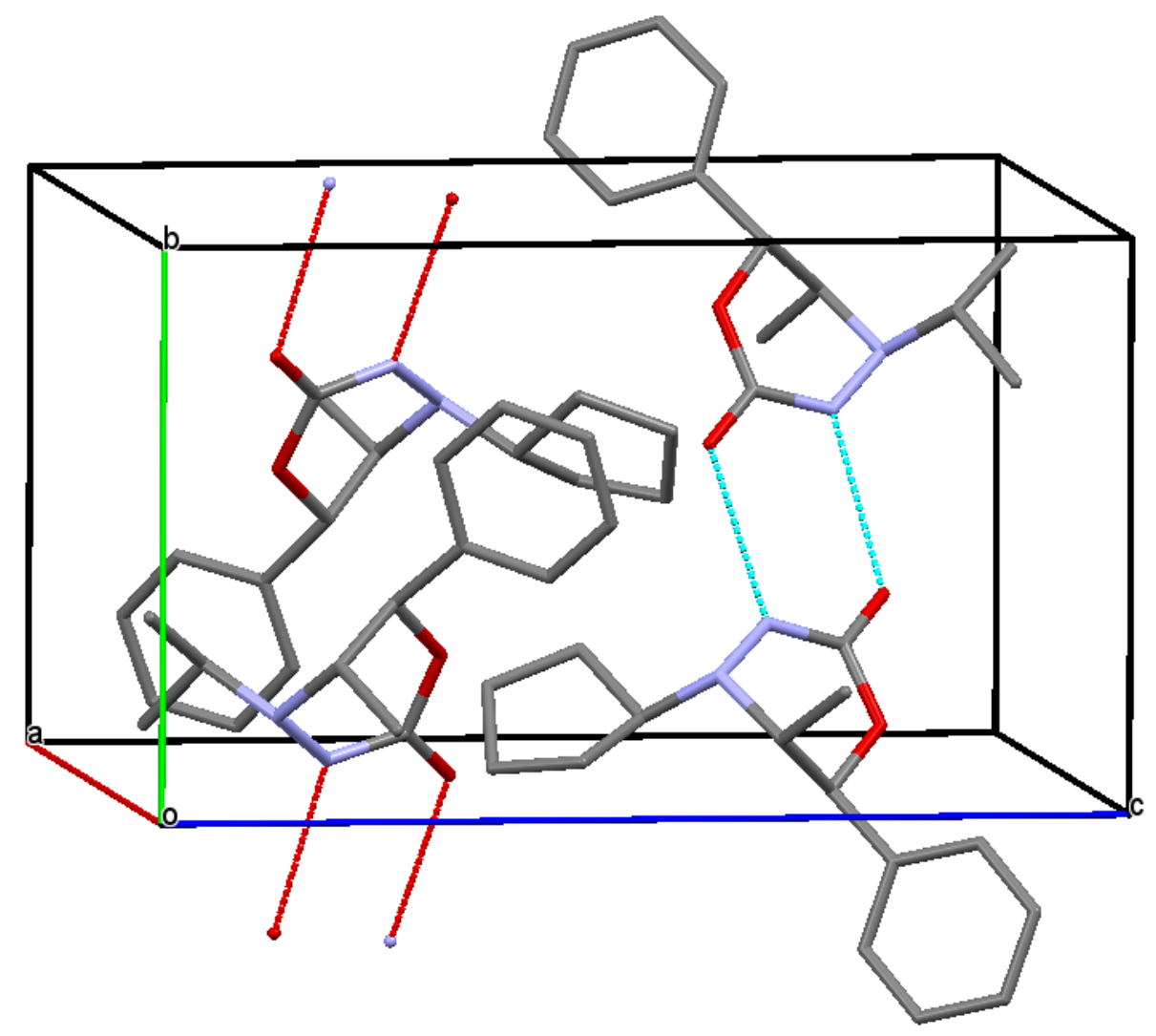

Figure 4.5 Packing diagram of 3. 
This quasiracemate compares to $\mathbf{8}$ (see Table 4.4) in that they both crystallize in the space group $P 2_{1}$. Both have two molecules in the asymmetric unit—one oxadiazinanone with an $\mathrm{N}_{4}$ cyclohexyl substituent and the other with an $\mathrm{N}_{4}$ substituent of isopropyl or cyclopentyl. This compound could possibly be modeled as a centrosymmetric crystal with disorder in the area of the isopropyl and cyclopentyl substituents with half occupancy of each of the substituents. Figure 4.6 shows an overlay of the quasienantiomers. The hydrogen atoms have been omitted for clarity.

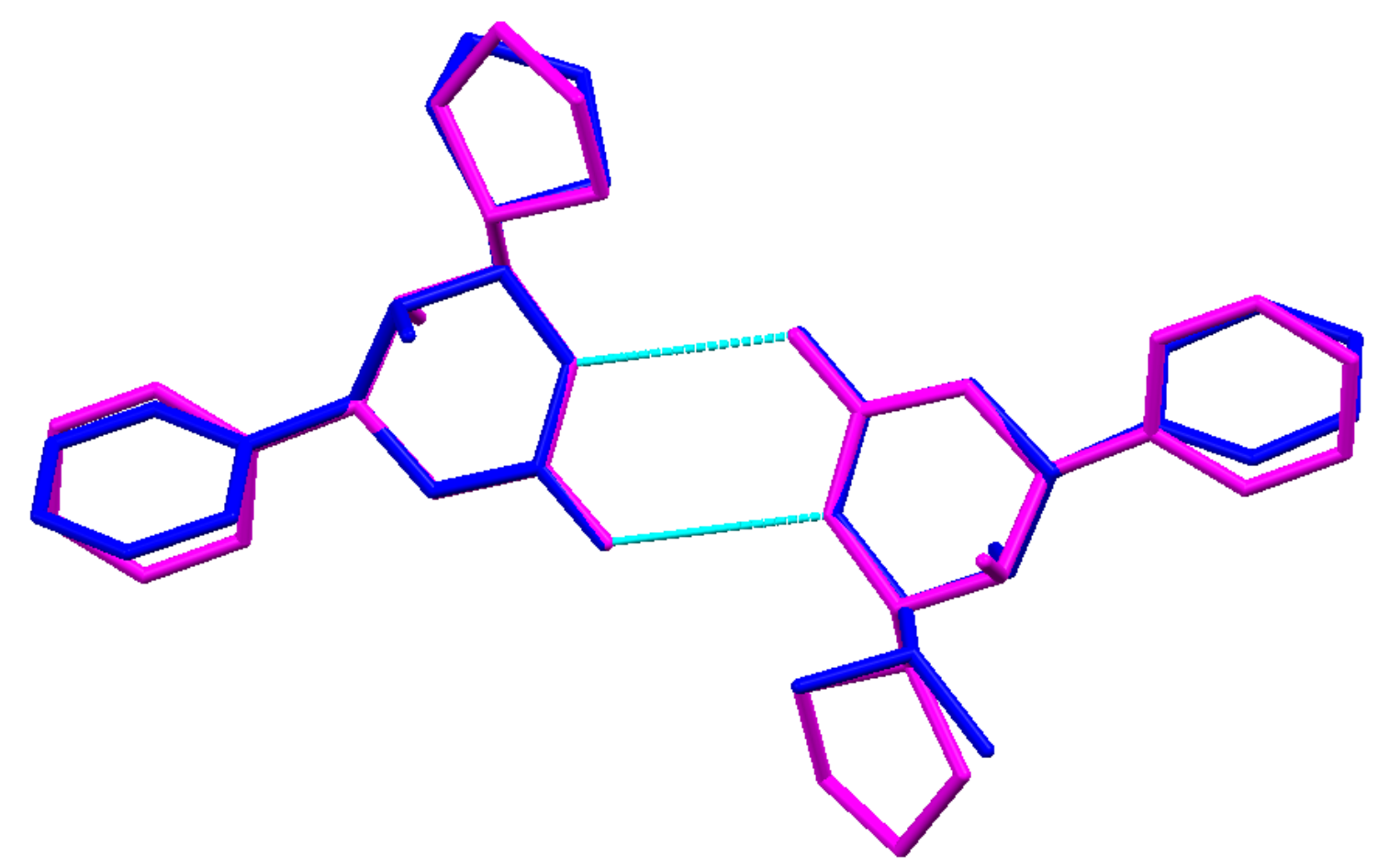

Figure 4.6 Overlay of 2 and 3.

In this overlay the nitrogen atoms and oxygen atoms used in the hydrogenbonding have been used to determine the least squares alignment of the structures. It is important to note that as one looks further from the center where the structures are 
aligned, the differences become more exaggerated. In this overlay it seems that the isopropyl substituent and the cyclopentyl constituent are closely aligned and that this could be solved in a centrosymmetric space group.

In Figure 4.7 one can compare the hydrogen-bonded dimers of 2 and 3. The similarities are striking —again making a case for the possibility of solving the structure in a centrosymmetric space group. The hydrogen atoms have been omitted for clarity.
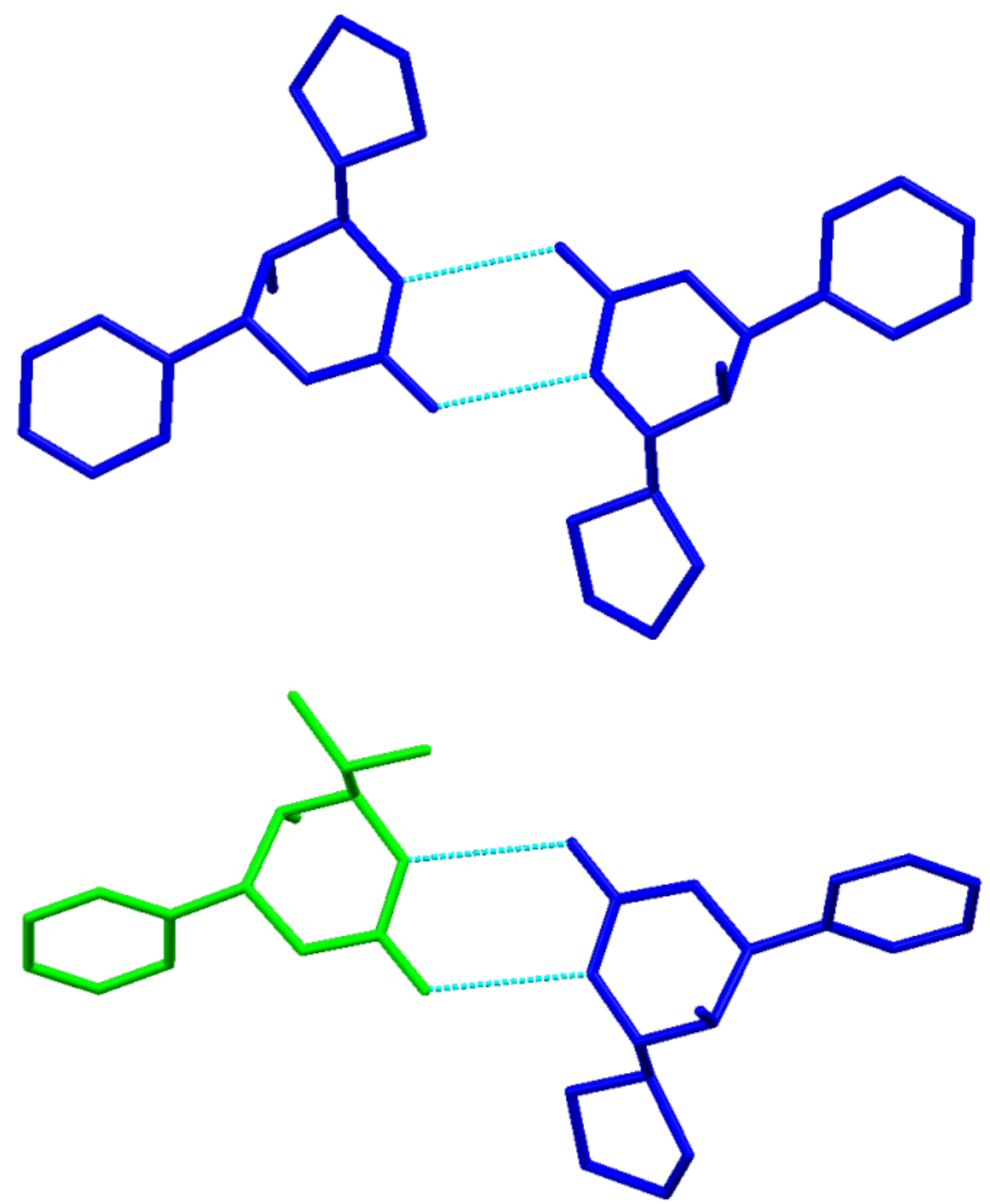

Figure 4.7 The hydrogen-bonded dimers of $\mathbf{2}$ (top) and $\mathbf{3}$ (bottom). 
Compound $\mathbf{2}$ has an inversion center located within the hydrogen bonds and, of course 3, does not. However, Compound $\mathbf{3}$ could have a pseudo-inversion center located within the hydrogen bonds. Figure 4.8 shows $\mathbf{2}$ and $\mathbf{3}$ from an angle which allows one to compare the hydrogen bonds. Again, hydrogen atoms have been omitted for clarity.

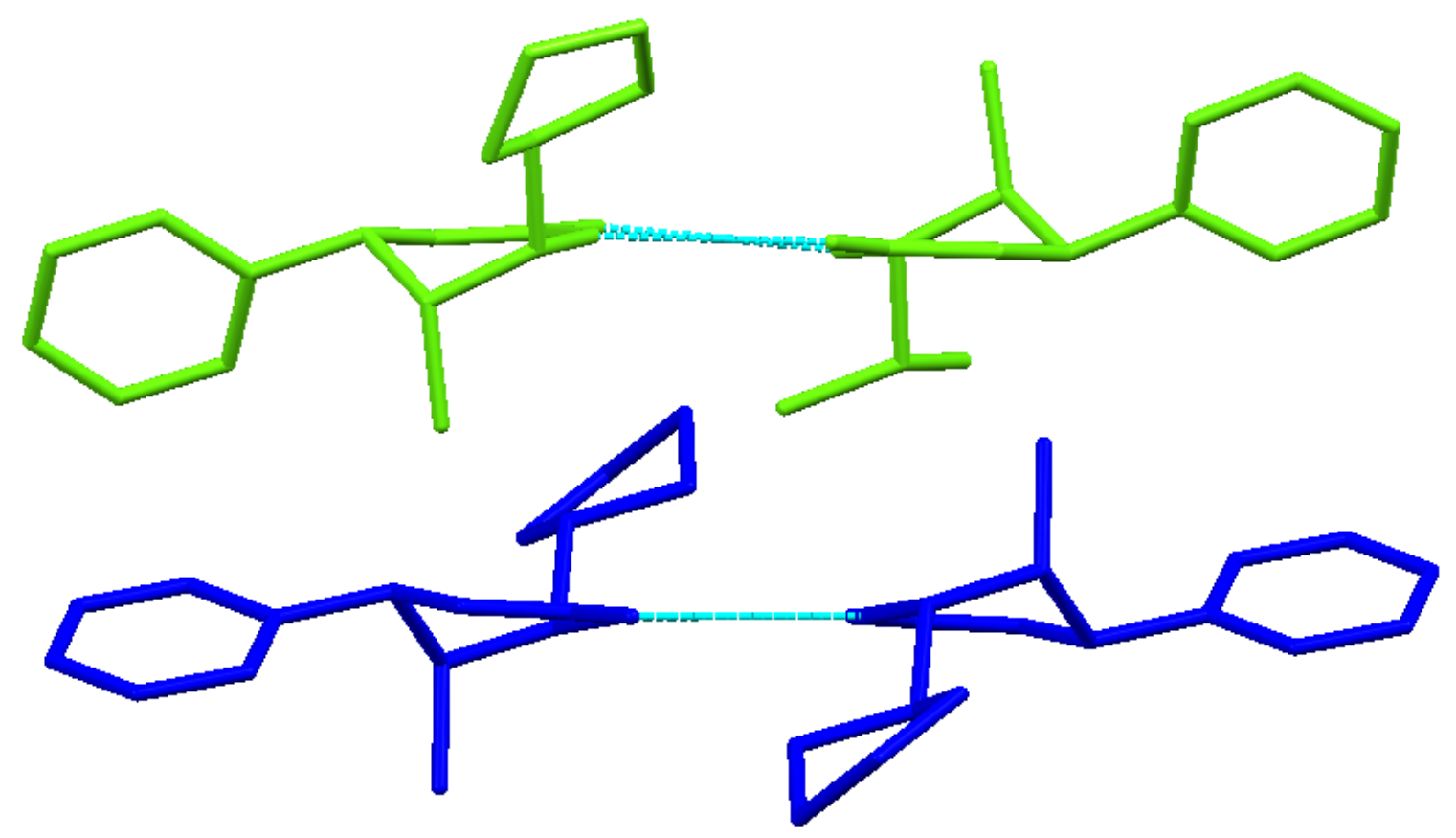

Figure 4.8 The upper structure is a wireframe depiction of the quasiracemate, $\mathbf{3}$, and the lower structure is that of the racemate, $\mathbf{1}$. Both structures are shown from a perspective parallel to the hydrogen-bonds.

In Figure 4.8 one can see that the hydrogen bonds in $\mathbf{1}$ appear parallel to each other and the molecule does not show much flexion in the bonds. In 3, the hydrogen bonds are skewed in slightly different directions, and there is some flexing of the different enantiomers. This is further evidenced in the difference in the torsion angles between the four atoms which are hydrogen bonded. In $\mathbf{1}$ the torsion angle is $0.00(5)^{\circ}$ and 
in 3 the torsion angle is $3.86(6)^{\circ}$. The distances between the nitrogen and oxygen atoms in the hydrogen bonds in 3 are 2.868(2) $\AA$ and 2.895(2) $\AA$. The distance between the nitrogen and oxygen atoms in the hydrogen bonds in 2 is 2.824(2) $\AA$. It can be seen, therefore, that there is more discrepancy between the structures than is immediately evident.

Analysis of the Quasiracemate of (5R,6S)-5-Methyl-4-cyclopentyl-6-phenyl-2H-1,3,4oxadiazinan-2-one and (5S,6R)-4-Cylohexyl-5-methyl-6-phenyl-2H-1,3,4oxadiazinan-2-one, 4

Compound 4 crystallizes in the monoclinic, chiral $P 2_{1}$ space group. There are two molecules in the asymmetric unit (see Figure 3.11). The only symmetry element present is a two-fold screw axis. As in 2, hydrogen bonding in $\mathbf{4}$ occurs between the two quasienantiomers in a hetero-enantiomorph motif. In Figure 4.9 one can see the packing of 4 in the unit cell. Hydrogen atoms have been omitted for clarity.

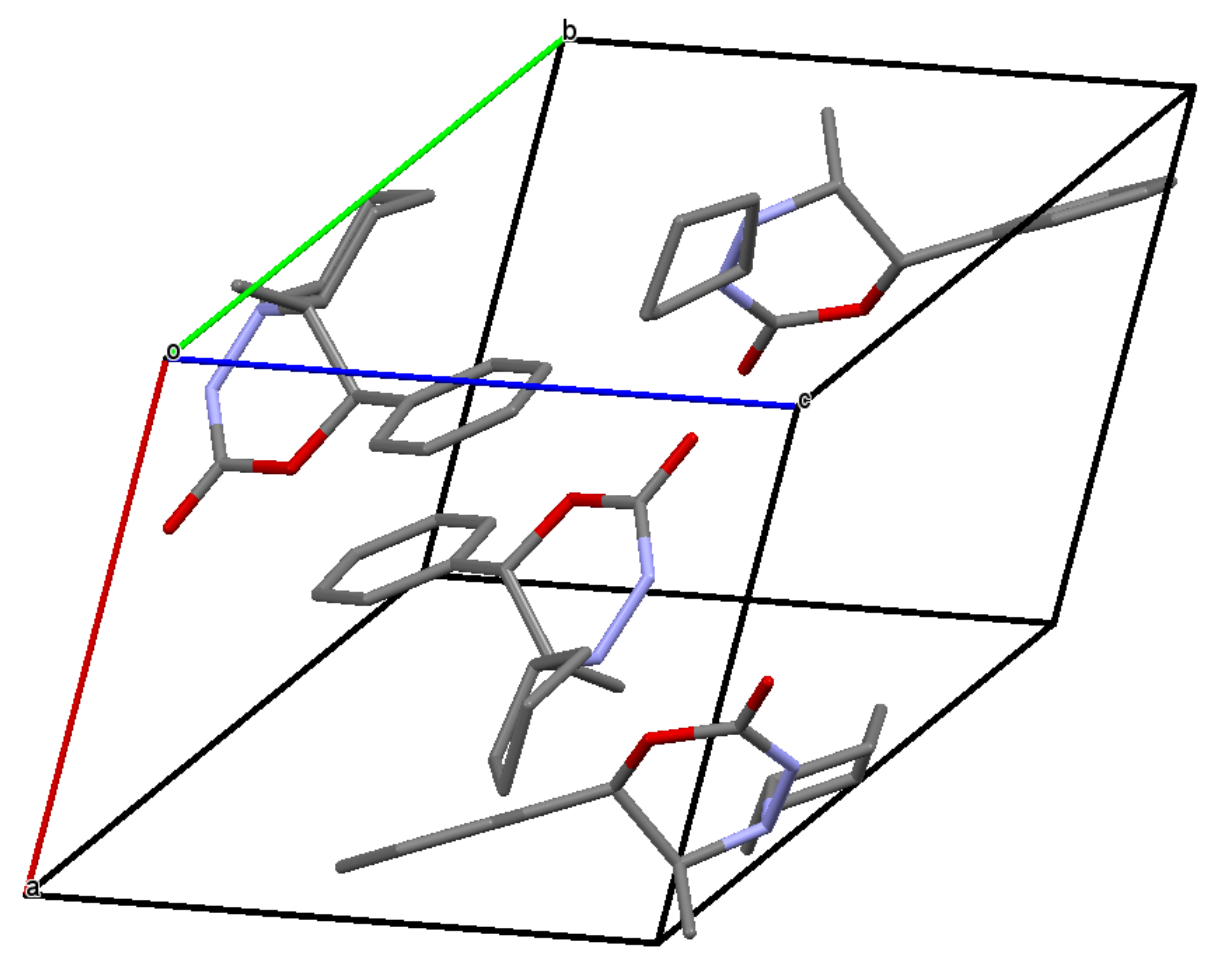

Figure 4.9 Packing diagram of the unit cell of 4 . 
In Figure 4.10 one can see the overlay of $\mathbf{2}$ (racemate) and $\mathbf{4}$ (quasiracemate). In this overlay the nitrogen atoms and oxygen atoms at each of the double bonds have been used in the least squares analysis. These atoms near the center of the structure are forced to be aligned as closely as possible which cause the differences to be exaggerated as one analyzes the differences further from the center. With that concept in mind, one can see the close alignment of the cyclopentyl group in the racemate and the cyclohexyl group in the quasiracemate.

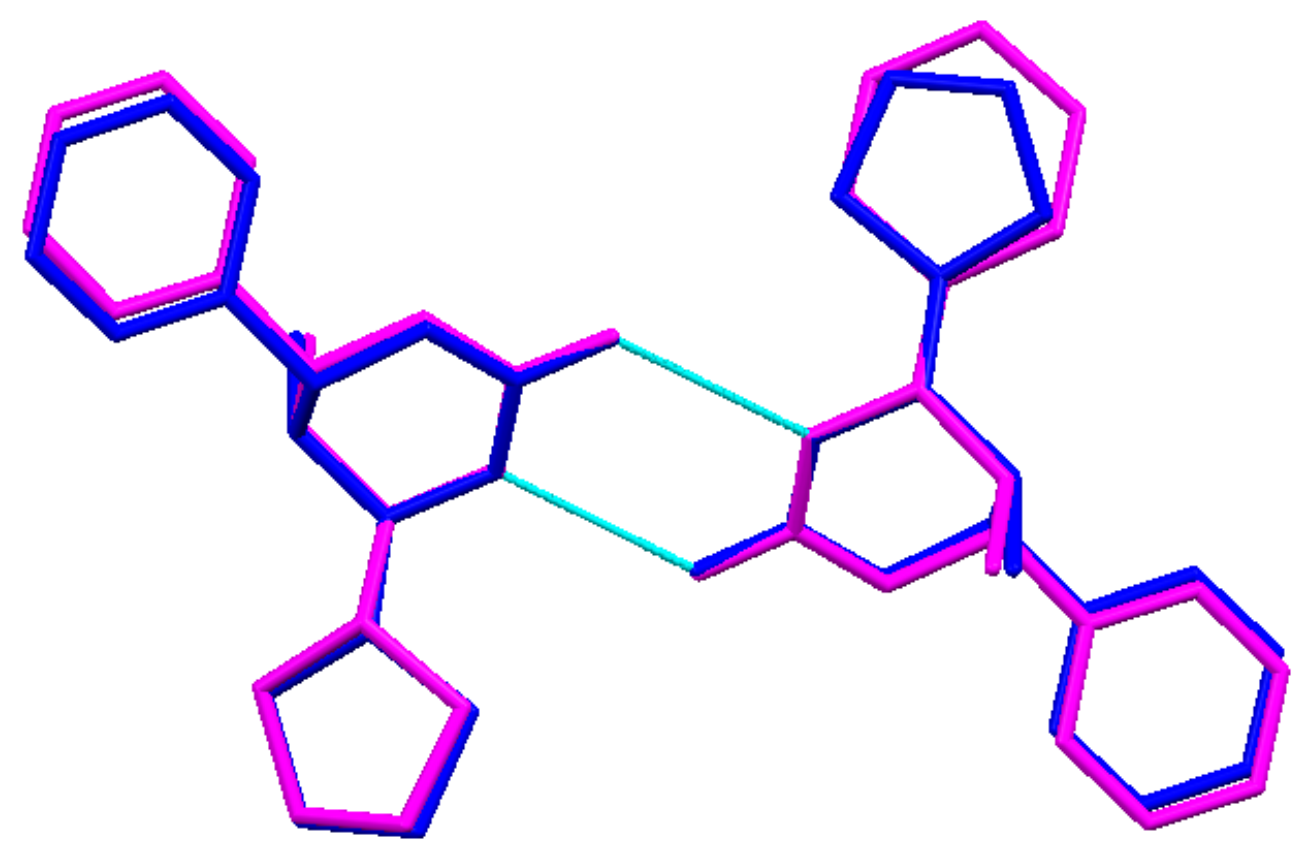

Figure 4.10 Overlay of 2 and 4.

In Figure 4.11 one can see that the hydrogen bonds in $\mathbf{2}$ and $\mathbf{4}$ are very similar and $\mathbf{4}$ seems to have little flexion. In $\mathbf{1}$ the torsion angle is $0.00(5)^{\circ}$ and in $\mathbf{3}$ the torsion angle is $0.52(9)^{\circ}$. The distances between the nitrogen and oxygen atoms in 4 are 2.859(3) $\AA$ and 
2.823(3) A. The distance between the nitrogen and oxygen atoms in each of the hydrogen bonds in the racemate 2 is $8.824(2) \AA$.

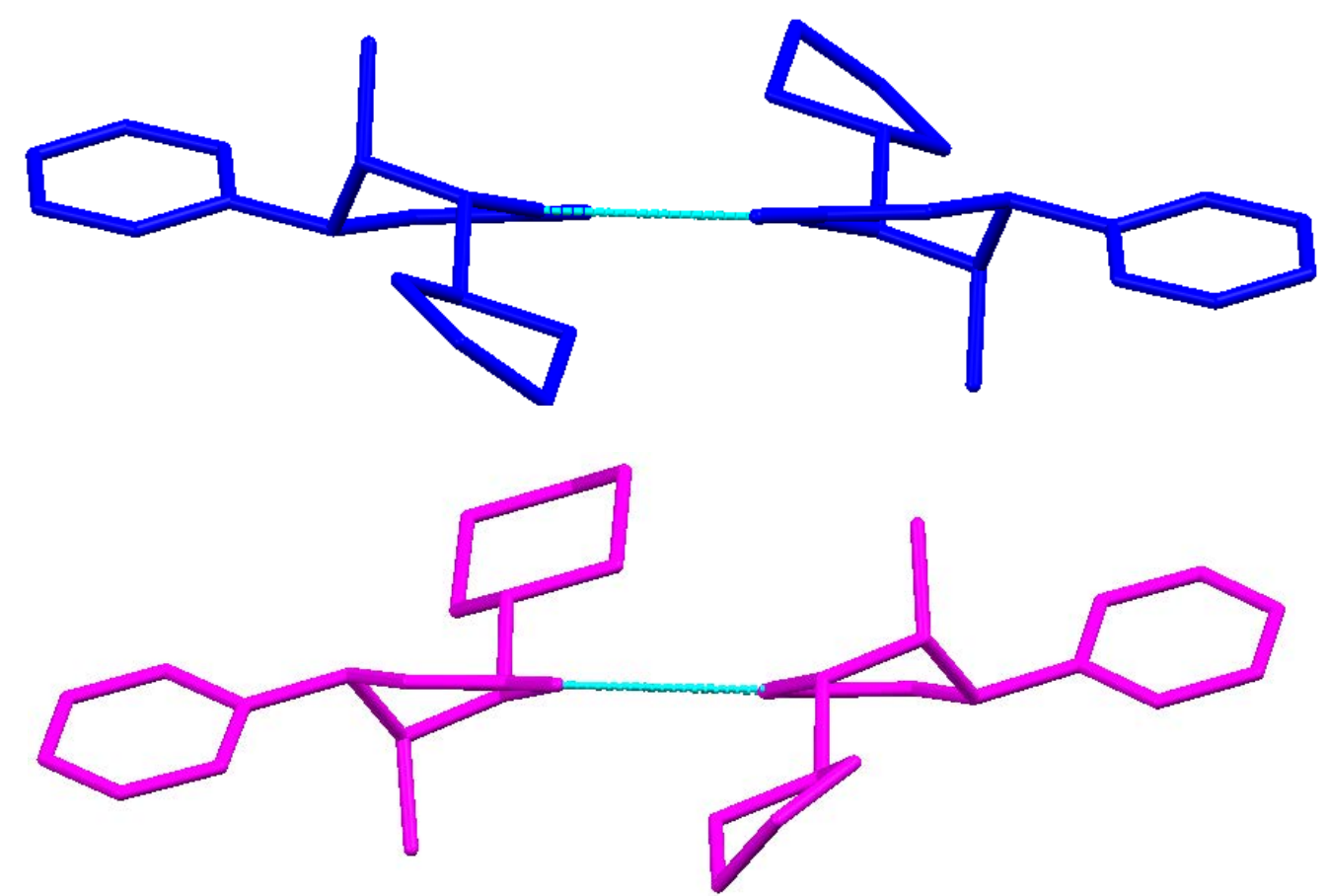

Figure 4.11 Hydrogen-bond comparison of 2 (top) and $\mathbf{4}$ (bottom).

The comparison of the hydrogen bond angles and torsion angles between $\mathbf{2}$ and $\mathbf{4}$ are closer than those between $\mathbf{2}$ and $\mathbf{3}$ as can be seen in Table 4.6.

Table 4.6 Torsion angles and bond distances in the hydrogen bond motifs of 2, $\mathbf{3}$ and $\mathbf{4}$.

\begin{tabular}{l|llll}
\hline \multicolumn{2}{c}{} & \begin{tabular}{c} 
Hydrogen Bond Distances \\
$(\AA)$ \\
\multicolumn{1}{c}{ Donor to Acceptor }
\end{tabular} & Atoms & $\begin{array}{c}\text { Torsion Angles } \\
\left({ }^{\circ}\right)\end{array}$ \\
\hline Compound 2 & $2.824(2) \AA$ & N3-O19-N3-19 & $0.00(5)$ \\
Compound 3 & $2.868(2) \AA ; 2.895(2) \AA$ & O69-N3-O17-N53 & $3.86(6)$ \\
Compound 4 & $2.859(3) \AA ; 2.823(3) \AA$ & O69-N5-O20-N53 & $0.52(9)$ \\
\hline
\end{tabular}


The similarity in the structure for racemic cyp and cyp/cyh leads one to wonder if the quasiracemate could be solved in a centrosymmetric space group modeling a disorder with the cyclohexyl quasiracemate and the cyclopentyl racemate present in equal amounts. This modelling was attempted and Figure 4.12 shows the resulting asymmetric unit.

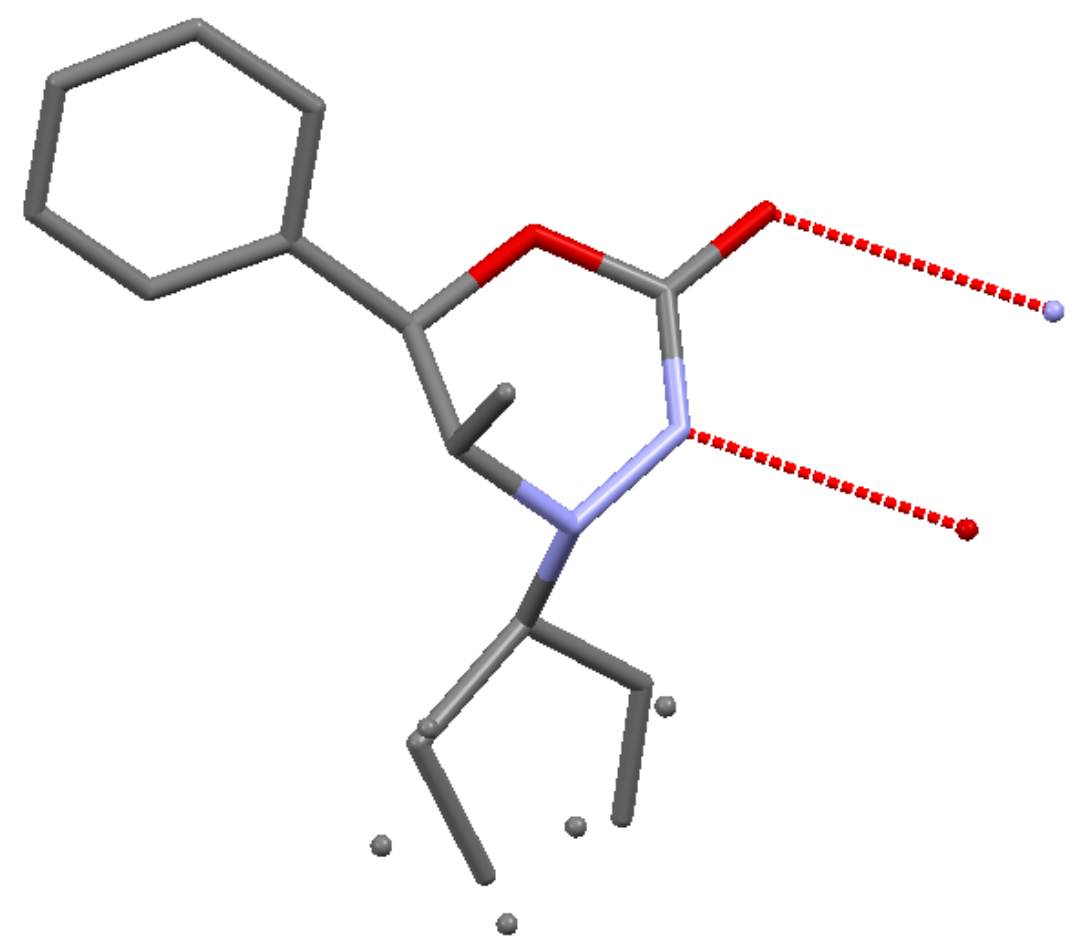

Figure 4.12 Asymmetric unit of 4 solved in $P 2_{1} / c$ space group.

In this structure the lengths of the hydrogen bonds are both 2.77(1) $\AA$. The torsion angles are $0.0^{\circ}$. These values are constrained because the second molecule is generated by an inversion. The extra atoms that can be seen around the $\mathrm{N}_{4}$ substituent represent the disorder at the $\mathrm{N}_{4}$ substituent. When solved in the centrosymmetric space group $P 2{ }_{1} / c, Z$ $=4$ and $Z^{\prime}=1$. The $R^{1}$-factor is 0.1635 and $w R^{2}$ is 0.3397 . The $\mathrm{R}$-factors are higher than what should be accepted in a structure, but it is conceivable that they are low enough that 
this could be used as a proof of concept. The definition of a quasiracemate could be changed to reflect the ability of the structure to be solved in a centrosymmetric space group with some higher level of $R$-values, weighted or un-weighted. This definition does not mean the structure should be solved and published as a centrosymmetric crystal, but that the structure is capable of being solved in a centrosymmetric space group. This also indicates that the $Z^{\text {‘ }}=2$ of $\mathbf{4}$ is a bit misleading, because it is much like $\mathbf{2}$ which has a $Z^{\prime}$ of 1 .

\section{Analysis of 5}

Compound 5 (Figure 3.13) was the result of an attempt to synthesize an oxadiazinanone substituted at the $\mathrm{N}_{4}$ position with an ethyl group. The cyclization reaction, after purification with flash chromatography, appeared to produce the desired product based on the ${ }^{1} \mathrm{H}$ NMR spectrum.

After solving the structure using X-ray diffraction, it was found that the $\mathrm{N}_{4}$ substituent was the ethyl group but the ring had not closed. It is possible that the desired product was produced in addition to the product which was identified by X-ray diffraction, but the oxadiazinanone was more soluble than the non-cyclized product and did not crystallize. Figure 4.13 gives the reaction for the synthesis of the non-cyclized product, $(1 S, 2 R)$ 2-[N-ethyl- $N$ '-o-ethyl hydrazinoate]-1-phenyl-propan-2-ol, 5, from the hydrazine of the ethyl derivative of $(1 S, 2 \mathrm{R})$ norephedrine. 
<smiles>CCOC(=O)NN(CC)[C@@H](C)[C@H](O)c1ccccc1</smiles>

Figure 4.13 An attempt to cyclize the hydrazine of the ethyl derivative of $(1 S, 2 R)$ norephedrine resulted in 5 .

Compound 5 crystallizes in the orthorhombic space group, $P 2{ }_{1} 2_{1} 2_{1}$, and has one molecule in the asymmetric unit $\left(Z^{\prime}=1\right)$. The compound has three 2 -fold screw axes as the only elements of symmetry. The asymmetric unit has two hydrogen bonds. Figure 3.14 shows that one of the hydrogen bonds is homoenantiomophic and one is an intramolecular bond. It is interesting to note that the hydroxyl group at $\mathrm{O} 13$ is a proton donor to the intramolecular carbonyl group and a proton acceptor from the secondary amine at N3. In Figure 4.14, it can be seen that there are four molecules in the unit cell, therefore $Z=4$.

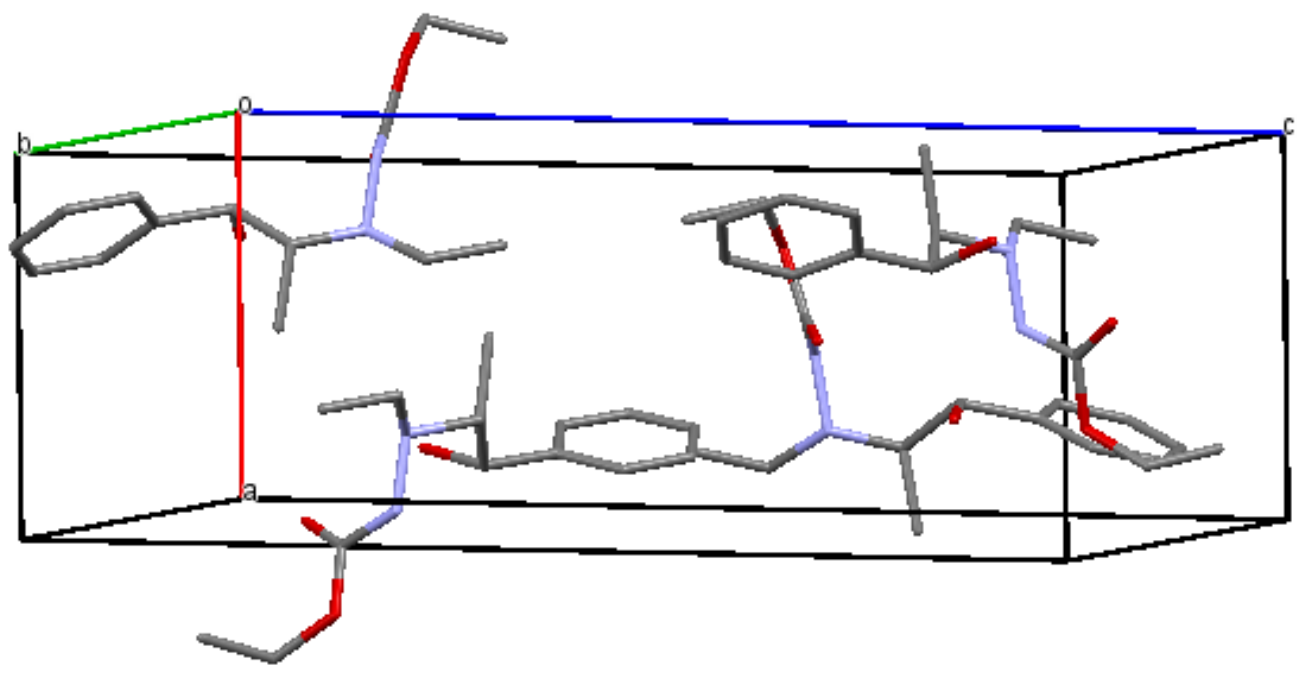

Figure 4.14 Packing diagram of the unit cell of 5. 


\section{Analysis of 6}

Compound 6 (Figure 3.14) was the result of an attempt to synthesize an oxadiazinanone substituted at the $\mathrm{N}_{4}$ position with an $n$-butyl group. The cyclization reaction, after purification with flash chromatography, appeared to produce the desired product based on the ${ }^{1} \mathrm{H}$ NMR spectrum.

After solving the structure using X-ray diffraction, it was found that the $\mathrm{N}_{4}$ substituent was the $n$-butyl group but the ring had not closed. It could be that the desired product was produced in addition to the product which was identified by X-ray diffraction, but the oxadiazinanone was more soluble than the non-cyclized product and did not crystallize. Figure 4.15 gives the reaction for the synthesis of the non-cyclized product, $(1 S, 2 R)$ 2-[N-n-butyl-N'-o-n-butyl hydrazinoate]-1-phenyl-propan-2-ol, 6, from the hydrazine of the $n$-butyl derivative of $(1 S, 2 R)$ norephedrine.

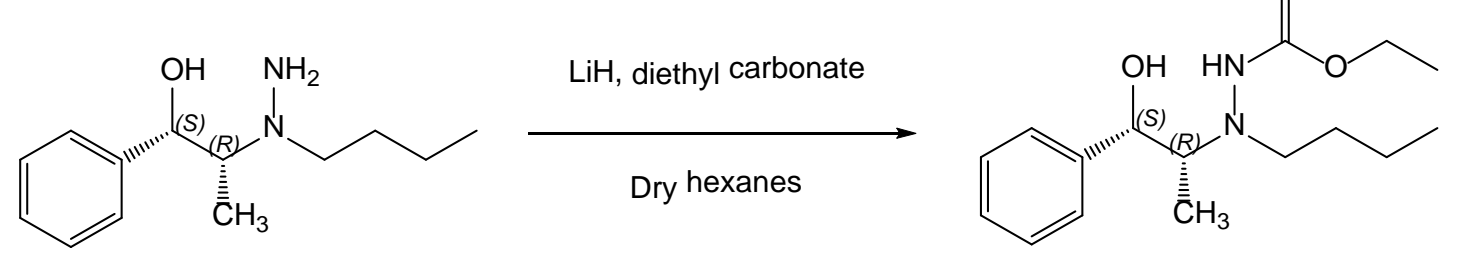

Figure 4.15 An attempt to cyclize the hydrazine of the $n$-butyl derivative of $(1 S, 2 R)$ norephedrine resulted in synthesis of $\mathbf{6}$.

The compound crystallizes in the monoclinic space group, $P 2_{1}$, and has two molecules in the asymmetric unit $\left(Z^{\prime}=2\right)$. The only symmetry element is a two-fold screw axis. This compound has only two hydrogen bonds. One of the bonds is intramolecular. The other is between a carbonyl group and an alcohol group on the other 
molecule in the asymmetric unit. Using symbols and terminology of Etter et al., ${ }^{14}$ this would have two motifs, S(6) and D, indicating the intramolecular bond (S) and the bond between the two molecules of the asymmetric unit (D).

Figure 4.16 shows the packing diagram of the unit cell. The two molecules, although the same compound, have different conformations. This is readily apparent when the two molecules are compared in Tables 3.22 and 3.23. The $n$-butyl group has enough flexion in the bonds and angles that perhaps the conformation changes enable the molecules to pack more densely.

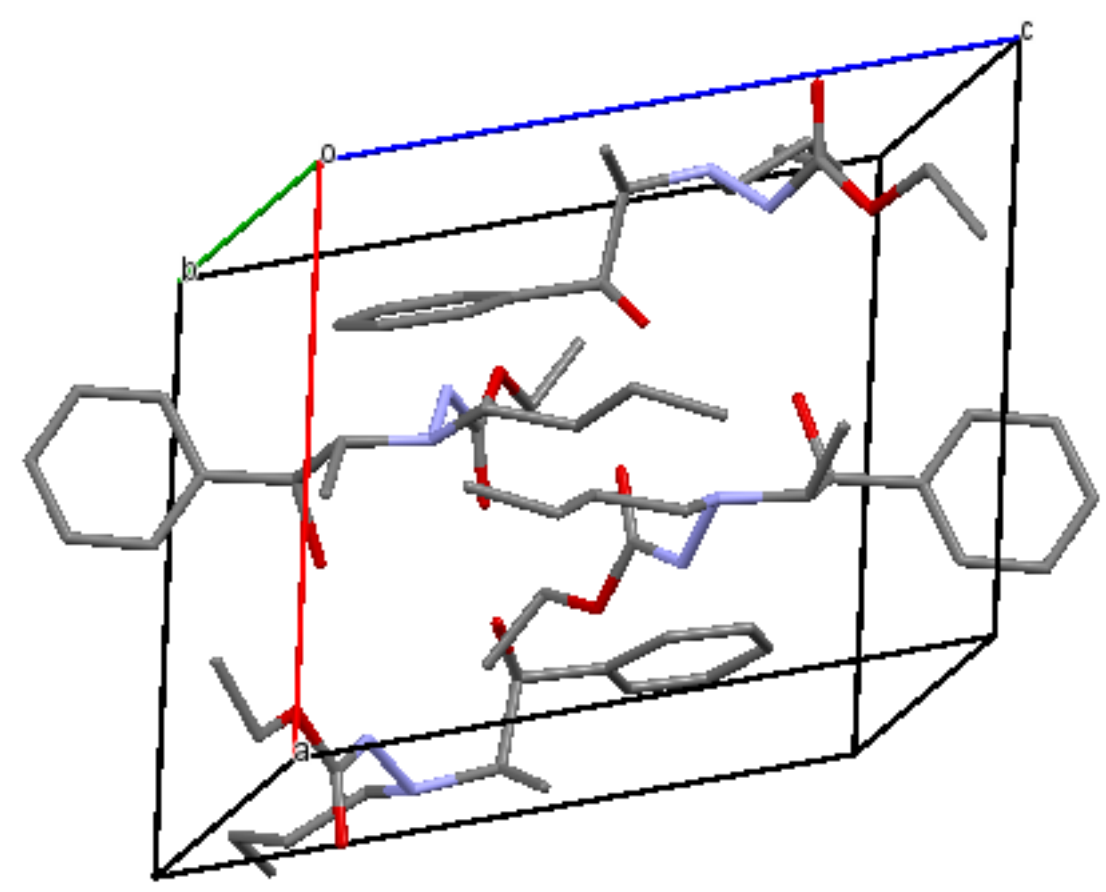

Figure 4.16 Packing diagram of the unit cell of 6.

Polymorphic transformation was displayed in $\mathbf{6}$. When the temperature of data collection of the $n$-butyl derivative was $150 \mathrm{~K}$, the crystal was monoclinic and when the temperature was lowered to $100 \mathrm{~K}$, the crystal morphed to a triclinic crystal. 
Crystallographic details could not be determined as the crystal shattered as it was cooled down. Polymorphic transformation can occur as the sizes of the atoms making up the compound increase or decrease based on the temperature. As the vibrations in the atom increase with increasing temperature, the increasing size of the molecule may make a less compact crystal more stable. ${ }^{47}$ The incidence of polymorphism is common if this statement of Walter C. McCrone is true: "Every compound has different polymorphic forms, and that, in general, the number of forms known for a given compound is proportional to the time and money spent in research on that compound.”48,49

In each of $\mathbf{5}$ and $\mathbf{6}$, the diethyl carbonate attached as expected to the amine group, but failed to attach at the hydroxyl group. The hydroxyl group has a stronger bond than the ammonia group, so the ammonia group left first and allowed the attachment of the diethyl carbonate. Changing the experimental procedure could produce the desired oxadiazinanone in each case. If the hexanes used as the solvent are not completely dry, the $\mathrm{LiH}$ will react with water more readily than with the hydrazine. Therefore, every effort must be made to ensure that the hexanes are dry. If the fourth step of the synthesis is repeated with the uncyclized product, the desired oxadiazinanone should be obtained. The LiH:hydrazine mole ratio was 1:2. Perhaps a greater concentration of LiH would also be effective in producing the oxadiazinanone.

\section{General Conclusions and Future Work}

At this point in the study of quasiracemates, the need is to acquire a database of structures. From this database it is hoped that the reasons for the formation of quasiracemic crystals with some compounds and not others can be elucidated. The knowledge gained from the study has the potential to direct the engineering of crystals to 
meet specific needs. To that end, this research can be helpful. The more data collected, the more knowledge may be gained about the reasons for quasiracemate cocrystallization.

The research goals for this project were met. The inventory of oxadiazinanones was increased. A new oxadiazinanone, (5R,6S)-4-cyclopentyl-5-methyl-6-phenyl-2H1,3,4-oxadiazinan-2-one, was synthesized. Attempts to combine oxadiazinanone enantiomers to form quasiracemates were made and two new quasiracemates were formed. Hydrogen bonding and the number of structures in the asymmetric unit were investigated as they related to the formation of the quasiracemates. The structure similarities of those quasienantiomers involved in quasiracemate formation were compared and an isopropyl type of connection to $\mathrm{N}_{4}$ seemed to be a common bond. How similar must the quasienantiomers be to co-crystallize? Further research with oxadiazinanones with different $\mathrm{N}_{4}$ substituents would be useful in determining this. Will oxadiazinanones form quasiracemates with oxadiazinathiones? Will the crystallization of oxadiazinathiones quasiracemates mirror that of oxadiazinanone compounds? The synthesis of oxadiazinanthiones with varying $\mathrm{N}_{4}$ substiuents and the subsequent combination of them with other oxadiazinanthiones or oxadiazinanones will provide new data. This data will perhaps provide new answers and probably raise some more questions. 


\section{REFERENCES}

1. Kelley, S.; Fabian, L.; Brock, C. Failures of Fractional Crystallization: Ordered Co-crystals of Isomers and Near Isomers. Acta Crystallogr., Sect. B: Struct. Sci. 2011, B67, 79-93.

2. $\quad$ Breen, M.; Tameze, S.; Dougherty, W.; Kassel, W.; Wheeler, K. Structural Studies of Enantiomers, Racemates, and Quasiracemates. 2-(3-Bromophenoxy)propionic Acid and 2-(3-Methoxyphenoxy)propionic Acid. Cryst. Growth Des. 2008, 8 (10), 38633870 .

3. Moss, G. P. Basic Terminology of Stereochemistry. Pure Appl. Chem. 1996, 68 (12), 2193-2222.

4. Husbye, S., An X-Ray Crystallographic Study of Some Racemates and Quasiracemates. Acta Chem. Scand. 1961, 15, 1215-1222.

5. Eliel, E.; Wilen, S. Stereochemistry of Organic Compounds; Wiley: New York, 1994.

6. Flack, H., Louis Pasteur's Discovery of Molecular Chirality and Spontaneous Resolution in 1848, Together with a Complete Review of his Crystallographic and Chemical Work. Acta Crystallogr., Sect. A: Found. Crystallogr. 2009, 65 (5), 371-389.

7. Zhang, Q.; Curran, D. Quansienantiomers and Quasiracemates: New Tools for Identrification, Analysis, Separation and Synthesis of Enantiomers. Chem. - Eur. J. 2005, 11, 4866-4880.

8. Denmark, S. E. Topics in Stereochemistry. John Wiley and Sons: New York, 1999; Vol. 22, p 336.

9. Mislow, K. Introduction to Stereochemistry. W.A.Benjamin: New York, 1965.

10. Centnerszwer, M. About Melting Points of Mixtures of Optical Antipodes. [machine translation] Z. Phys. Chem. 1899, 29, 715-725.

11. Fomulu, S.; Mukta, S.; Davis, R.; Wheeler, K. Structural Studies of Enantiomers, Racemates, and Quasiracemates. 2-(2,4,5-Trichloroanilino)propanoic Acid and 2-(2,4,5Trichlorophenoxy)propanoic Acid. Cryst. Growth Des. 2002, 2 (6), 637-644. 
12. Fredga, A. Steric Correlations by the Quasi-racemate Method. Tetrahedron 1960, $8,126-144$.

13. Karle, I.; Karle, J. The Crystal Structure of the Quasi-Racemate from (+)-mMethoxyphenoxypropionic Acid and (-)-m-Bromophenoxypropionic Acid. J. Am. Chem. Soc. 1966, 88 (1), 24-27.

14. Etter, M.; MacDonald, J.; Bernstein, J. Graph-Set Analysis of Hydrogen-Bond Patterns in Organic Crystals. Acta Crystallogr., Sect. B: Struct. Sci. 1990, B46, 256-262.

15. Sands, D. Introduction to Crystallography. Dover Publications, Inc.: Mineola, New York, 1975, p 165.

16. Hitchcock, S.; Nora, G.; Casper, D.; Squire, M.; Maroules, C.; Ferrence, G.;; Szczepura, L.; Standard, J. X-Ray Crystallographic and ${ }^{13} \mathrm{C}$ Nuclear Magnetic Resonance Studies of 3,4,5,6-Tetrahydro-2- $H$-1,3,4-oxadiazin-2-ones Derived from Ephedrine and Pseudoephedrine. Tetrahedron 2001, 57, 9789-9798.

17. Casper, D.; Blackburn J.; Maroules, C.; Brady, T.; Ferrence, G.;Standard, J.; Hitchcock, S. Conformational Studies of $N_{3}$-substituted [1,3,4]-Oxadiazinan-2-ones. $J$. Org. Chem. 2002, 67, 8871-8876.

18. Burgeson, J.; Renner, M.; Hardt, I.; Ferrence, G.; Standard, J.; Hitchcock, S. Towards the Development of a Structurally Novel Class of Chiral Auxiliaries. Conformational Properties of the Aldol Adducts of Oxadiazinones: Observation of Unusual Shielding Effects. J. Org. Chem. 2004, 69, 727-734.

19a. Knott, S.; Hitchcock, S.; Ferrence, G.; (5R,6S)-4,5-Dimethyl-3-methylacryloyl-6phenyl-1,3,4-oxadiazinan-2-one. Acta Crystallogr., Sect. E: Struct. Rep. Online 2008, E64, 1101.

19b. Addison, L.; Dore, D.; Hitchcock, S. (4R,5S)-5-Benzyl-4-isopropyl-1,3,4oxadiazinan-2-one. Acta Crystallogr., Sect. E: Struct. Rep. Online 2008, E64, 1040-1041.

20. Tailor, D.; Edler, K.; Casper, D.; Hitchcock, S.; Ferrence, G., (5S,6R)-4Isopropyl-5-methyl-6-phenyl-3-propanoyl-2H-1,3,4-oxadiazinan-2-one. Acta Crystallogr., Sect. E: Struct. Rep. Online 2009, E, 1685-1686.

21. Kocher, J.; Edler, K.; Bohling, B.; Nora, G.; Stafford, C.; Hitchcock, S.; Ferrence, G.; (5S,6R)-5-Methyl-6-phenyl-4-propyl-1,3,4-oxadiazinane-2-thione. Acta Crystallogr., Sect. E: Struct. Rep. Online 2009, E65, 1421-1422.

22. IUPAC, Compendium of Chemical Terminology, 2nd Ed. (the "Gold Book". McNaught, A.; Wilkinson, A., Eds. Blackwell Scientific Publications: Oxford, 1997.

23. Powell, G.; Revision of the Extended Hantzsch-Widman System of Nomenclature for Heteromonocycles. Pure Appl. Chem. 1983, 55, 409-416. 
24. Casper, D.; Nora, G.; Blackburn, J.; Bentley, J.; Taylor, D.; Hitchcock, S.

Synthesis of $N_{4}$-Substituted [1,3,4]Oxadiazinan-2-ones Derived from Norephedrine. $J$.

Heterocycl. Chem. 2002, 39, 823-828.

25. Casper, D.; Burgeson, J.; Esken, J.; Ferrence, G.; Hitchcock, S. Toward the Development of a Structurally Novel Class of Chiral Auxiliaries: Diastereoselective Aldol Reactions of a $(1 R, 2 S)$-Ephedrine Based 3,4,5,6-Tetrahydro-2H-1,3,4-oxadiazin-2one. Org. Lett. 2002, 4, 3739-3742.

26. Edler, Kate. M.S. Thesis, Illinois State University, Normal, IL, 2011.

27. Hitchcock, S.; Davis, R.; Richmond, D.; Dore, D.; Kuschel, S.; Vaughn, J. Synthesis, Asymmetric Aldol Reactions, and X-Ray Crystallography of some Oxadiazinanone Derivatives. J. Heterocycl. Chem. 2008, 45, 1265-1274.

28. Bentley, Jeremy. M.S. Thesis, Illinois State University, Normal, IL, 2002.

29. Hitchcock, S.; Nora, G.; Hedberg, C.; Casper, D.; Buchanan, L.; Squire, M., XRay Crystallographic and Proton Nuclear Magnetic Resonance Studies of beta-HydroxyN-nitrosamines derived from alpha-Amino Acids and Ephedrine. Tetrahedron 2000, 56, 8799-8807.

30. ChemDraw Corporation ChemDraw Std, version 13.0.2.3021. Cambridge, MA, 2013.

31. Spingler, B.; Schnidrig, S.; Todorova, T.; Wild, F. Some Thoughts About the Single Crystal Growth of Small Molecules. Cryst. Eng .Comm. 2012, 14, 751-757.

32. Bruker AXS SAINT AND SMART+, Madison, WI, 2003.

33. Farrugia, L. J. WinGX Suite for Small-Molecule Single-Crystal Crystallography. J. Appl. Crystallogr. 1999, 32 (4), 837-838.

34. Burla, M.; Caliandro, R.; Camalli, M.; Carrozzini, B.; Cascarano, G.; DeCaro, L., SIR2004:An improved Tool for Crystal Structure Determination and Refinement. J. Appl. Crystallogr. 2005, 38 (2), 381-388.

35. Palatinus, L.; Chapuis, G. SUPERFLIP- A Computer Program for the Solution of Crystal Structures by Charge Fipping in Arbitrary Dimensions. J. Appl. Crystallogr. 2007, 40, 786-790.

36. Sheldrick, G. M. SHELXS97 and SHELXL97, University of Göttingen, Germany, 1997.

37. Palatinus, L.; Chapuis, G. SUPERFLIP - A Computer Program for the Solution of Crystal Structures by Charge Flipping in Arbitrary Dimensions. J. Appl. Crystallogr. 2007, (40), 786-790. 
38. Szczepura, L.; Hitchcock, S.; Nora, G. (5S,6S)-3,4,5,6,-Tetrahydro-5-methyl-5phenyl-4-propyl-2H-1,3,4-oxadiazin-2-one. Acta Crystallogr., Sect. E: Struct. Rep. Online 2004, E60, 1467-1469.

39. Brock, C. Past and Present. Presented at Midwest Organic Solid State Chemistry Symposium XXIII, University of Kentucky, Lexington, KY, 2013.

40. Brock, C. P.; Dunitz, J. D. Towards a Grammar of Crystal Packing. Chem. Mater. 1994, 6 (8), 1118-1127.

41. Braun, B.; Kalf, I.; Englert, U. One of the Most Complex "Small Molecule Structures" Ever Reported: 16 Independent Molecules in the Asymmetric Unit for an ortho-Palladataed Primary Amine. Chem. Commun. 2011, 47, 3846-3848.

42. Brock, C.; Schweizer, W.; Dunitz, J. D. On the Validity of Wallach's Rule: On the Density and Stability of Racemic Crystals Compared with Their Chiral Counterparts. J. Am. Chem. Soc. 1991, 113, 9811-9820.

43. Springuel, G.; Norberg, B.; Robeyns, K.; Wouters, J.; Leyssens, T. Advances in Pharmaceutical Co-crystal Screening: Effective Co-crystal Screening through Structural Resemblance. Crys. Growth Des. 2011, 12, 475-484.

44. Steed, J., Should Solid-State Molecular Packing Have to Obey the Rules of Crystallographic Symmetry? CrystEngComm 2003, 5, 169-179.

45. MacRae, C.; Bruno, I.; Chisholm, J.; Edgington, P.; McCabe, P.; Pidcock, E.; Rodriquez-Monge, L.; Taylor, R.; van de Streek, J.; Wook, P., Mercury CSD 2.0-New Features for the Visualization and Investigation of Crystal Structures. J. Appl. Crystallogr. 2008, 41 (2), 466-470.

46. Clegg, W. Introduction to Symmetry and Diffraction. In Crystal Structure Analysis: Principles and Practice, 2 ed.; Clegg, W., Ed. Oxford University Press: New York, 2009; p 18.

47. Nelson, S. Twinning, Polymorphism, Polytypism, Pseudomorphism. http://www.tulane.edu/ sanelson/eens211/twinning.htm (accessed May, 16).

48 Liang, J. K. Small Molecule Crystallization, Illinois Institute of Technology, D. o. C. E., Ed. 2003;

49 McCrone, W. Polymorphism. In Physics and Chemistry of the Organic Solid State, Fox, D.; Labes, M.; Weissberger, A., Eds. Wiley-Interscience: New York, 1965; Vol. 2, pp 725-767. 